\title{
POTENTIALS OF A FAMILY OF ARRANGEMENTS OF HYPERPLANES AND ELEMENTARY SUBARRANGEMENTS
}

\begin{abstract}
Andrew Prudhom
A dissertation submitted to the faculty at the University of North Carolina at Chapel Hill in partial fulfillment of the requirements for the degree of Doctor of Philosophy in the Department of Mathematics.
\end{abstract}

Chapel Hill

2019
Approved by:

Alexander Varchenko

Prakash Belkale

Jiuzu Hong

Richard Rimanyi

Lev Rozansky 
(c) 2019

Andrew Prudhom

ALL RIGHTS RESERVED 


\section{ABSTRACT \\ Andrew Prudhom: Potentials of a family of arrangements of hyperplanes and elementary subarrangements \\ (Under the direction of Alexander Varchenko)}

We consider the Frobenius algebra of functions on the critical set of the master function of a weighted arrangement of hyperplanes in $\mathbb{C}^{k}$ with normal crossings. We construct two potential functions (of first and second kind) of variables labeled by hyperplanes of the arrangement and prove that the matrix coefficients of the Grothendieck residue bilinear form on the algebra are given by the $2 k$-th derivatives of the potential function of first kind and the matrix coefficients of the multiplication operators on the algebra are given by the $(2 k+1)$-st derivatives of the potential function of second kind. Thus the two potentials completely determine the Frobenius algebra. The presence of these potentials is a manifestation of a Frobenius like structure similar to the Frobenius manifold structure.

We introduce the notion of an elementary subarrangement of an arrangement with normal crossings. It turns out that our potential functions are local in the sense that the potential functions are sums of contributions from elementary subarrangements of the given arrangement. This is a new phenomenon of locality of the Grothendieck residue bilinear form and multiplication on the algebra.

It is known that this Frobenius algebra of functions on the critical set is isomorphic to the Bethe algebra of this arrangement. (That Bethe algebra is an analog of the Bethe algebras in the theory of quantum integrable models.) Thus our potential functions describe that Bethe algebra too. 


\section{TABLE OF CONTENTS}

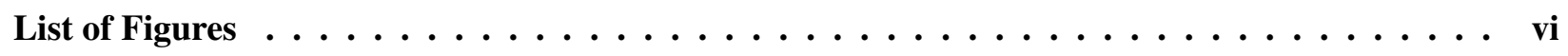

Introduction $\ldots \ldots \ldots \ldots \ldots \ldots \ldots \ldots \ldots \ldots \ldots \ldots \ldots \ldots \ldots$

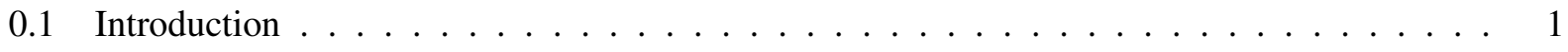

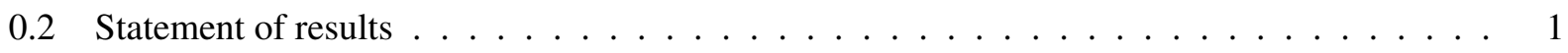

0.3 Frobenius like structure of $\operatorname{order}(n, k, m) \ldots \ldots \ldots \ldots$

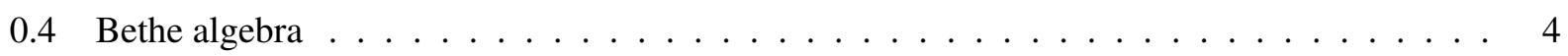

0.5 Exposition of material $\ldots \ldots \ldots \ldots \ldots \ldots \ldots \ldots \ldots \ldots \ldots \ldots \ldots \ldots \ldots \ldots \ldots$

Chapter 1. Arrangements .......................... 6

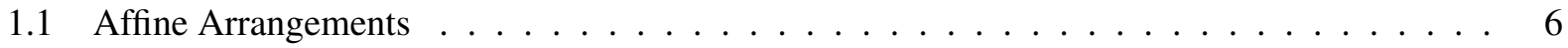

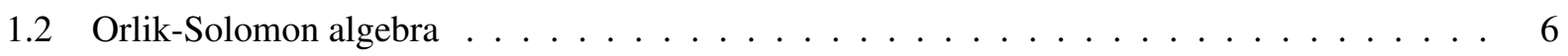

1.3 Aomoto complex $\ldots \ldots \ldots \ldots \ldots \ldots \ldots$

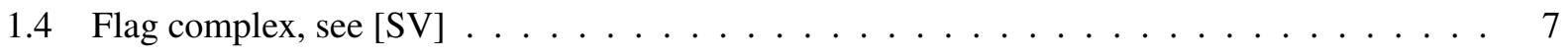

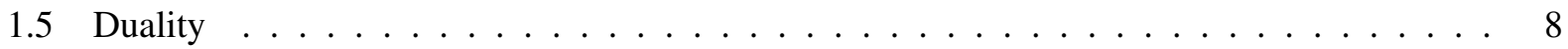

1.6 Contravariant map and form, see $[\mathrm{SV}] \ldots \ldots \ldots \ldots \ldots$

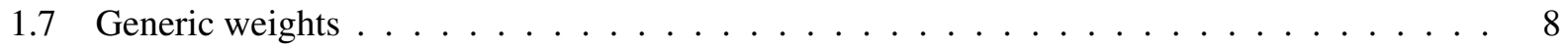

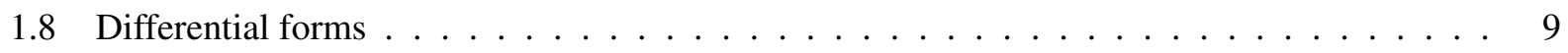

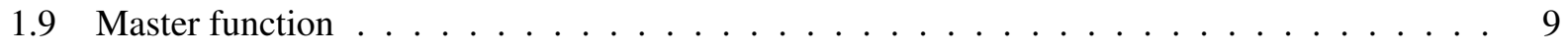

1.10 Isolated critical points $\ldots \ldots \ldots \ldots \ldots \ldots \ldots$

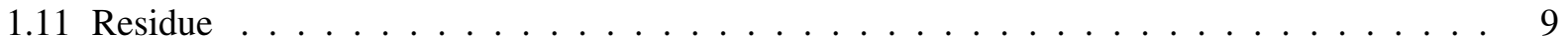

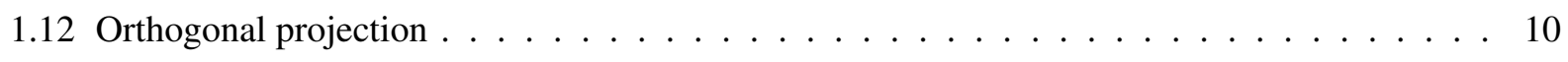

1.13 Integral structure on $\mathcal{O}\left(C_{\mathcal{C}, a}\right)$ and $\operatorname{Sing}_{a} \mathcal{F}^{k}(\mathcal{C}) \ldots \ldots \ldots \ldots$

1.14 Combinatorial connection, $\mathrm{I} \ldots \ldots \ldots \ldots \ldots \ldots$

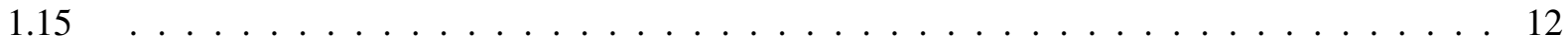


Chapter 2. Family of parallelly transported hyperplanes . . . . . . . . . . . . . . 14

2.1 Arrangement in $\mathbb{C}^{n} \times \mathbb{C}^{k} \ldots \ldots \ldots \ldots \ldots \ldots \ldots \ldots$

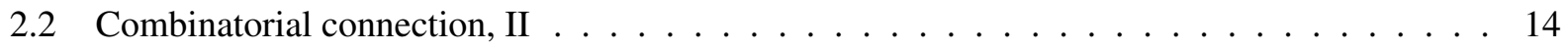

2.3 Gauss-Manin connection on $\left(\mathbb{C}^{n}-\Delta\right) \times\left(\operatorname{Sing}_{a} V\right) \rightarrow \mathbb{C}^{n}-\Delta \ldots \ldots \ldots$

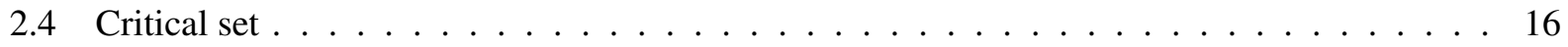

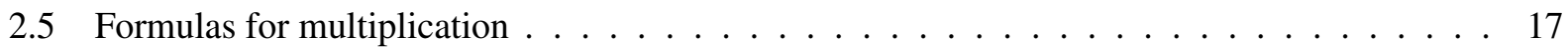

Chapter 3. Elementary arrangements . . . . . . . . . . . . . . . . 19

3.1 Definition . . . . . . . . . . . . . . . . . . . . . . 19

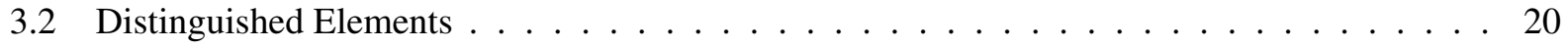

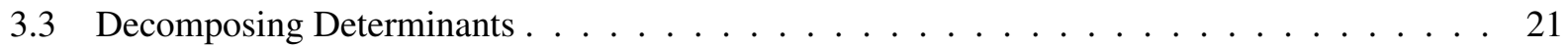

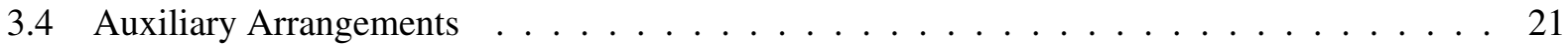

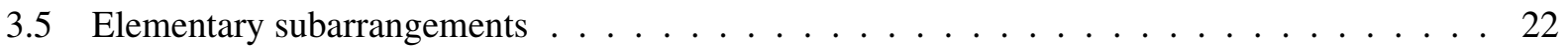

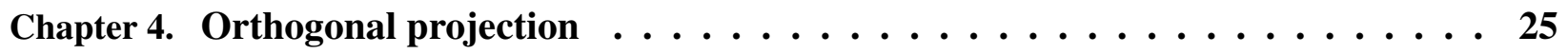

4.1 Formula for orthogonal projection $\ldots \ldots \ldots \ldots \ldots$

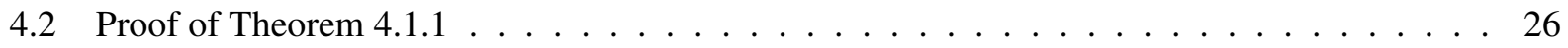

4.3 Potential of first kind $\ldots \ldots \ldots \ldots \ldots \ldots$

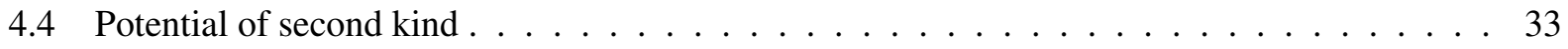

Chapter 5. Unit Element for 2 and 3-Dimensional Arrangements . . . . . . . . . . 37

5.1 Formula for the Unit Element of $A_{\Phi}$ in 2-Dimensions $\ldots \ldots \ldots$

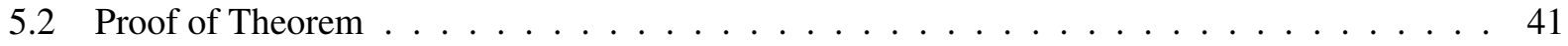

5.3 Conjectural Formula for 3-Dimensional Unit Element $\ldots \ldots \ldots \ldots$

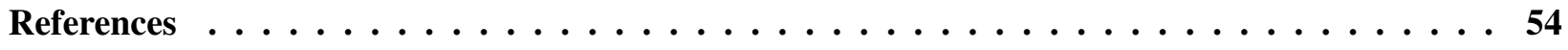




\section{LIST OF FIGURES}

1 An arrangement of lines in 2 Dimensions $\ldots \ldots \ldots \ldots$

2 Elementary Arrangements in Dimension 2. . . . . . . . . . . . . . . . . . . . 19 


\section{INTRODUCTION}

\subsection{Introduction}

It is well known that the algebra of functions on the set of solutions of the Bethe ansatz equations plays an important role in the study of quantum integrable systems since in many cases the algebra of functions is isomorphic to the Bethe algebra of Hamiltonians of the system, see for example [NS, MTV1, GRTV, R]. An interesting problem is to describe the algebra. In this paper we consider the model case of the algebra of functions on the critical set of the master function associated with a family of arrangements with normal crossings. Such algebras appear in the KZ-Gaudin type integrable systems, see for example [SV, RV]. We describe the algebra of functions on the critical set together with the Grothendieck residue bilinear form in terms of derivatives of two potential functions in the spirit of Frobenius structures.

\subsection{Statement of results}

Denote $J=\{1, \ldots, n\}$. Consider $\mathbb{C}^{n} \times \mathbb{C}^{k}$ with coordinates $(z, t)=\left(z_{1}, \ldots, z_{n}, t_{1}, \ldots, t_{k}\right)$ and the projection $\tau: \mathbb{C}^{n} \times \mathbb{C}^{k} \rightarrow \mathbb{C}^{n}$. Fix $n$ nonzero linear functions on $\mathbb{C}^{k}, g_{j}=b_{j}^{1} t_{1}+\cdots+b_{j}^{k} t_{k}, j \in J$. Assume that $\left\{g_{j}\right\}_{j \in J}$ span the dual space $\left(\mathbb{C}^{k}\right)^{*}$. Define the functions $f_{j}=g_{j}+z_{j}$ on $\mathbb{C}^{n} \times \mathbb{C}^{k}$. We obtain on $\mathbb{C}^{n} \times \mathbb{C}^{k}$ an arrangement $\mathcal{C}=\left\{H_{j}\right\}_{j=1}^{n}$, where $H_{j}$ is the zero set of $f_{j}$. Let $U(\mathcal{C}):=\mathbb{C}^{n} \times \mathbb{C}^{k}-\cup_{j \in J} H_{j}$ be the complement. For every $x \in \mathbb{C}^{n}$, the arrangement $\mathcal{C}$ restricts to an arrangement $\mathcal{C}(x)$ on $\tau^{-1}(x) \cong \mathbb{C}^{k}$ with the complement $U(\mathcal{C}(x)):=\tau^{-1}(x) \cap U(\mathcal{C})$. For almost all $x \in \mathbb{C}^{k}$ the arrangement $\mathcal{C}(x)$ is with normal crossings. The subset $\Delta \subset \mathbb{C}^{n}$, where this does not hold, is a hypersurface and is called the discriminant.

A set $I=\left\{i_{1}, \ldots, i_{k}\right\} \subset J$ is called independent if $g_{i_{1}}, \ldots, g_{i_{k}}$ are linearly independent. Denote $J^{\text {ind }}$ the set of all independent $k$-element subsets of $J$.

Let $a=\left(a_{1}, \ldots, a_{n}\right) \in\left(\mathbb{C}^{*}\right)^{n}$ be a system of weights such that for any $x \in \mathbb{C}^{n}-\Delta$ the weighted arrangement $(\mathcal{C}(x), a)$ is unbalanced, see Section 1.10, e.g. $a \in \mathbb{R}_{>0}^{n}$ is unbalanced, also a generic system of 
weights is unbalanced. The master function of the weighted arrangement $(\mathcal{C}, a)$ is

$$
\Phi_{\mathcal{C}, a}(z, t):=\sum_{i \in J} a_{i} \log f_{i}
$$

For $x \in \mathbb{C}^{n}-\Delta$ all critical points of $\left.\Phi_{\mathcal{C}, a}\right|_{z=x}$ with respect to the variables $t$, are isolated, and the sum $\mu$ of their Milnor numbers is independent of the unbalanced weight $a$ and the parameter $x \in \mathbb{C}^{n}-\Delta$. The main object of this paper is the $\mu$-dimensional algebra

$$
\mathcal{O}\left(C_{\mathcal{C}(x), a}\right):=\mathcal{O}(U(\mathcal{C}(x))) /\left(\frac{\partial \Phi_{\mathcal{C}, a}}{\partial t_{j}} \mid j=1, \ldots, k\right)
$$

of functions on the critical set of the master function $\left.\Phi_{\mathcal{C}, a}\right|_{z=x}$, see Section 2.4. Define

$$
p_{j}:=\left[\frac{a_{j}}{f_{j}}\right]=\left[\frac{\partial \Phi_{\mathcal{C}, a}}{\partial z_{j}}\right] \in \mathcal{O}\left(C_{\mathcal{C}(x), a}\right), \quad j \in J
$$

The elements $\left\{p_{j}\right\}_{j \in J}$ generate $\mathcal{O}\left(C_{\mathcal{C}(x), a}\right)$ as an algebra. The elements $\left\{p_{i_{1}} \cdots p_{i_{k}}\right\}_{\left\{i_{1}, \ldots, i_{k}\right\}} \in J^{\text {ind }}$ generate $\mathcal{O}\left(C_{\mathcal{C}(x), a}\right)$ as a vector space. The Grothendieck residue defines a nondegenerate bilinear form $(,)_{C_{\mathcal{C}(x), a}}$ on $\mathcal{O}\left(C_{\mathcal{C}(x), a}\right)$. The algebra $\left(\mathcal{O}\left(C_{\mathcal{C}(x), a}\right),(,)_{C_{\mathcal{C}(x), a}}\right)$ is a Frobenius algebra.

The main result of this paper is a construction of two functions $P, Q$ on $\mathbb{C}^{n}-\Delta$ called the potentials of first and second kind, respectively. The potentials have the following properties.

Theorem 0.2.1. Let $x \in \mathbb{C}^{n}-\Delta$. Then for any two independent subsets $\left\{i_{1}, \ldots, i_{k}\right\},\left\{l_{1}, \ldots, l_{k}\right\} \subset J$ and any $i_{0} \in J$, we have

$$
\begin{gathered}
\left(p_{i_{1}} \cdots p_{i_{k}}, p_{l_{1}} \cdots p_{l_{k}}\right)_{C_{\mathcal{C}(x), a}}=(-1)^{k} \frac{\partial^{2 k} P}{\partial z_{i_{1}} \ldots \partial z_{i_{k}} \partial z_{l_{1}} \ldots \partial z_{l_{k}}}(x), \\
\left(p_{i_{0}} p_{i_{1}} \cdots p_{i_{k}}, p_{l_{1}} \cdots p_{l_{k}}\right)_{C_{\mathcal{C}(x), a}}=(-1)^{k} \frac{\partial^{2 k+1} Q}{\partial z_{i_{0}} \partial z_{i_{1}} \ldots \partial z_{i_{k}} \partial z_{l_{1}} \ldots \partial z_{l_{k}}}(x) .
\end{gathered}
$$

Formula (4) determines the Grothendieck residue bilinear form $(,)_{C_{\mathcal{C}(x), a}}$ in terms of the potential of first kind. Formula (5) determines the operators of multiplication by generators $\left\{p_{j}\right\}_{j \in J}$ in terms of the potential of second kind. 


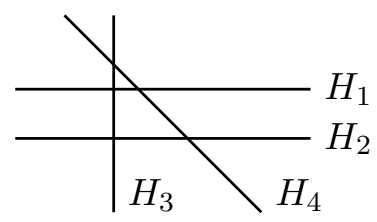

Figure 1: An arrangement of lines in 2 Dimensions

Example 1. For the arrangement of four lines shown in Figure 1 and given by equations $t_{2}+z_{1}=0$, $t_{2}+z_{2}=0, t_{1}+z_{3}=0, t_{1}+t_{2}+z_{4}=0$ we have

$$
\begin{gathered}
P=\frac{1}{a_{1}+a_{2}+a_{3}+a_{4}}\left(a_{1} a_{3} a_{4} \frac{\left(z_{1}+z_{3}-z_{4}\right)^{4}}{4 !}+a_{2} a_{3} a_{4} \frac{\left(z_{2}+z_{3}-z_{4}\right)^{4}}{4 !}\right. \\
\left.+\frac{a_{1} a_{2} a_{3} a_{4}}{a_{3}+a_{4}} \frac{\left(z_{1}-z_{2}\right)^{2}}{2 !} \frac{\left(z_{1}+z_{3}-z_{4}\right)^{2}}{2 !}\right), \\
Q=a_{1} a_{3} a_{4} \ln \left(z_{1}+z_{3}-z_{4}\right) \frac{\left(z_{1}+z_{3}-z_{4}\right)^{4}}{4 !}+a_{2} a_{3} a_{4} \ln \left(z_{2}+z_{3}-z_{4}\right) \frac{\left(z_{2}+z_{3}-z_{4}\right)^{4}}{4 !} \\
+\frac{a_{1} a_{2} a_{3} a_{4}}{a_{3}+a_{4}} \ln \left(z_{1}-z_{2}\right) \frac{\left(z_{1}-z_{2}\right)^{2}}{2 !} \frac{\left(z_{1}+z_{3}-z_{4}\right)^{2}}{2 !} .
\end{gathered}
$$

Theorem 0.2.1 in particular says that $\left(p_{1} p_{3}, p_{2} p_{4}\right)_{C_{\mathcal{C}(x), a}}=\frac{a_{1} a_{2} a_{3} a_{4}}{\left(a_{1}+a_{2}+a_{3}+a_{4}\right)\left(a_{3}+a_{4}\right)}$ and it does not depend on $x \in \mathbb{C}^{n}-\Delta$, and $\left(p_{4} p_{1} p_{3}, p_{3} p_{4}\right)_{C_{\mathcal{C}(x), a}}=\frac{a_{1} a_{3} a_{4}}{z_{1}+z_{3}-z_{4}}$.

In this example the potentials are sums of terms corresponding to subarrangements consisting of three or four lines. It turns out that this is the general case. In Section 3.1 we introduce the notion of an elementary arrangement in $\mathbb{C}^{k}$ of type $\lambda=\left(\lambda_{1}, \ldots, \lambda_{m}\right), \lambda_{h} \in \mathbb{Z}_{>0}, \lambda_{1}+\cdots+\lambda_{m}=k$. In particular, such an elementary arrangement consists of $k+m$ hyperplanes, and an elementary arrangement in $\mathbb{C}^{k}$ has at most $2 k$ hyperplanes. We show that the potentials are sums, over all elementary subarrangements, of the prepotentials of the subarrangements taken with suitable weights, see Corollary 4.3.4 and Theorem 4.4.1. The fact that the potentials are sums of contributions from elementary subarrangements indicates a new phenomenon of locality of the Grothendieck residue bilinear form and multiplication on $\mathcal{O}\left(C_{\mathcal{C}(x), a}\right)$.

The existence of the potentials of first and second kind locally on $\mathbb{C}^{n}-\Delta$ was established in [HV]. 


\subsection{Frobenius like structure of order $(n, k, m)$}

The potential of the second kind is an analog of the potential in the theory of Frobenius manifolds. A Frobenius manifold is a manifold with a flat metric and a Frobenius algebra structure on tangent spaces at points of the manifold such that the structure constants of multiplication are given by third derivatives of a potential function on the manifold with respect to flat coordinates, see [D, M]. As an analogy of that, for our family of arrangements the structure constants of multiplication are given by $2 k+1$-st derivatives of the potential of second kind, see Theorem 0.2.1.

The notion of potentials of a family of arrangements was introduced and studied in [V5, V7, HV]. In [V5] the potentials were constructed for the families of generic arrangements, that is, such that the linear functions $g_{i_{1}}, \ldots, g_{i_{k}}$ are linearly independent for any distinct $i_{1}, \ldots, i_{k} \in J$. In [V5, V7, HV] different axiomatizations of the structure leading to the existence of the potentials were given. In particular in [HV] Frobenius like structures of order $(n, k, m)$ were introduced. Our case of a family of arrangements corresponds to the case of order $(n, k, 2)$. Under the axioms of [HV] the existence of the potential of second kind was deduced in [HV] from a surprising elementary study of finite sets of vectors in a finite-dimensional vector space $W$. Given a natural number $m$ and a finite set $\left\{w_{i}\right\}$ of vectors, a necessary and sufficient condition was given to find in the set $\left\{w_{i}\right\} m$ bases of $W$. If $m$ bases in the set $\left\{w_{i}\right\}$ are selected, then some elementary transformations of such a selection are defined. It was shown in [HV] that any two selections are connected by a sequence of elementary transformations. These structures are fundamental and one may expect a matroid version of them.

\subsection{Bethe algebra}

Given a family of weighted arrangements in $\mathbb{C}^{k}$ as in Section 0.2 , one considers the Gauss-Manin differential equations for associated $k$-dimensional hypergeometric integrals, $\kappa \frac{\partial I}{\partial z_{j}}(z)=K_{j}(z) I(z), j \in J$, $z \in \mathbb{C}^{n}-\Delta$, where $K_{i}(z)$ are suitable linear operators on the space of singular vectors $\operatorname{Sing}_{a} V$, see Section 2.3. For every $x \in \mathbb{C}^{n}-\Delta$, the operators $K_{j}(x), j \in J$, commmute and are symmetric with respect to the contravariant bilinear form $S^{(a)}$ on $\operatorname{Sing}_{a} V$. The unital subalgebra of $\operatorname{End}\left(\operatorname{Sing}_{a} V\right)$ generated by the operators $K_{j}(x), j \in J$, is called the Bethe algebra of the weighted arrangement $(\mathcal{C}(x), a)$. This algebra is the analog of the Bethe algebra in the theory of quantum integrable systems, see [V4]. It is known that the Bethe algebra together with the bilinear form $S^{(a)}$ is isomorphic to the pair consisting of the algebra of 
multiplication operators on $\mathcal{O}\left(C_{\mathcal{C}(x), a}\right)$ and the Grothendieck residue bilinear form $(,)_{C_{\mathcal{C}(x), a}}$. Thus Theorem 0.2.1 gives us a description of the Bethe algebra in terms of the derivatives of the potential functions, see Theorem 4.3.1 and Corollary 4.4.4.

Our construction of potential functions is based on the isomorphism of the Bethe algebra and the algebra of functions on the critical set.

The Bethe algebra of our family of arrangements is a toy example of the Bethe algebra of a quantum integrable system. One may expect to determine glimpses of Frobenius like structures in the Bethe algebras of standard quantum integrable systems.

\subsection{Exposition of material}

In Chapter 1 we remind general facts about arrangements. In Chapter 2 we consider families of arrangements. In Chapter 3 we introduce elementary arrangements and define potential functions. In Chapter 4 we prove an important formula for the orthogonal projection $\pi: V \rightarrow \operatorname{Sing}_{a} V$ with respect to the bilinear form $S^{(a)}$. Based on that formula we prove the first part of Theorem 0.2.1 in Section 4.3 and the second part of Theorem 0.2.1 in Section 4.4. 


\section{CHAPTER 1}

\section{Arrangements}

\subsection{Affine Arrangements}

Let $k, n$ be positive integers, $k<n$. Denote $J=\{1, \ldots, n\}$.

Consider the complex affine space $\mathbb{C}^{k}$ with coordinates $t_{1}, \ldots, t_{k}$. Let $\mathcal{C}=\left(H_{j}\right)_{j \in J}$, be an arrangement of $n$ affine hyperplanes in $\mathbb{C}^{k}$. Denote $U(\mathcal{C})=\mathbb{C}^{k}-\cup_{j \in J} H_{j}$, the complement. An edge $X_{\alpha} \subset \mathbb{C}^{k}$ of $\mathcal{C}$ is a nonempty intersection of some hyperplanes of $\mathcal{C}$. Denote by $J_{\alpha} \subset J$ the subset of indices of all hyperplanes containing $X_{\alpha}$. Denote $l_{\alpha}=\operatorname{codim}_{\mathbb{C}^{k}} X_{\alpha}$. We assume that $\mathcal{C}$ is essential, that is, $\mathcal{C}$ has a vertex, an edge which is a point.

An edge is called dense if the subarrangement of all hyperplanes containing it is irreducible: the hyperplanes cannot be partitioned into nonempty sets so that, after a change of coordinates, hyperplanes in different sets are in different coordinates. In particular, each hyperplane of $\mathcal{C}$ is a dense edge.

\subsection{Orlik-Solomon algebra}

Define complex vector spaces $\mathcal{A}^{p}(\mathcal{C}), p=0, \ldots, k$. For $p=0$, we set $\mathcal{A}^{p}(\mathcal{C})=\mathbb{C}$. For $p \geqslant 1, \mathcal{A}^{p}(\mathcal{C})$ is generated by symbols $\left(H_{j_{1}}, \ldots, H_{j_{p}}\right)$ with $j_{i} \in J$, such that (i) $\left(H_{j_{1}}, \ldots, H_{j_{p}}\right)=0$ if $H_{j_{1}}, \ldots, H_{j_{p}}$ are not in general position, that is, if the intersection $H_{j_{1}} \cap \ldots \cap H_{j_{p}}$ is empty or has codimension less than $p$; (ii) $\left(H_{j_{\sigma(1)}}, \ldots, H_{j_{\sigma(p)}}\right)=(-1)^{|\sigma|}\left(H_{j_{1}}, \ldots, H_{j_{p}}\right)$ for any element $\sigma$ of the symmetric group $\Sigma_{p}$; (iii) $\sum_{i=1}^{p+1}(-1)^{i}\left(H_{j_{1}}, \ldots, \widehat{H}_{j_{i}}, \ldots, H_{j_{p+1}}\right)=0$ for any $(p+1)$-tuple $H_{j_{1}}, \ldots, H_{j_{p+1}}$ of hyperplanes in $\mathcal{C}$ which are not in general position and such that $H_{j_{1}} \cap \ldots \cap H_{j_{p+1}} \neq \emptyset$.

The direct sum $\mathcal{A}(\mathcal{C})=\oplus_{p=1}^{N} \mathcal{A}^{p}(\mathcal{C})$ is the Orlik-Solomon algebra with respect to multiplication $\left(H_{j_{1}}, \ldots, H_{j_{p}}\right) \cdot\left(H_{j_{p+1}}, \ldots, H_{j_{p+q}}\right)=\left(H_{j_{1}}, \ldots, H_{j_{p}}, H_{j_{p+1}}, \ldots, H_{j_{p+q}}\right)$. 


\subsection{Aomoto complex}

Fix a point $a=\left(a_{1}, \ldots, a_{n}\right) \in\left(\mathbb{C}^{\times}\right)^{n}$ called the weight. Then the arrangement $\mathcal{C}$ is weighted: for $j \in J$, we assign weight $a_{j}$ to hyperplane $H_{j}$. For an edge $X_{\alpha}$, define its weight $a_{\alpha}=\sum_{j \in J_{\alpha}} a_{j}$. We denote $a_{J}=\sum_{j \in J} a_{j}$ and $\omega^{(a)}=\sum_{j \in J} a_{j} \cdot\left(H_{j}\right) \in \mathcal{A}^{1}(\mathcal{C})$. Multiplication by $\omega^{(a)}$ defines the differential $d^{(a)}: \mathcal{A}^{p}(\mathcal{C}) \rightarrow \mathcal{A}^{p+1}(\mathcal{C}), x \mapsto \omega^{(a)} \cdot x$, on $\mathcal{A}(\mathcal{C})$.

\subsection{Flag complex, see [SV]}

For an edge $X_{\alpha}, l_{\alpha}=p$, a flag starting at $X_{\alpha}$ is a sequence $X_{\alpha_{0}} \supset X_{\alpha_{1}} \supset \cdots \supset X_{\alpha_{p}}=X_{\alpha}$ of edges such that $l_{\alpha_{j}}=j$ for $j=0, \ldots, p$. For an edge $X_{\alpha}$, we define $\left(\overline{\mathcal{F}}_{\alpha}\right)_{\mathbb{Z}}$ as the free $\mathbb{Z}$-module generated by the elements $\bar{F}_{\alpha_{0}, \ldots, \alpha_{p}=\alpha}$ labeled by the elements of the set of all flags starting at $X_{\alpha}$. We define $\left(\mathcal{F}_{\alpha}\right)_{\mathbb{Z}}$ as the quotient of $\left(\overline{\mathcal{F}}_{\alpha}\right)_{\mathbb{Z}}$ by the submodule generated by all the elements of the form

$$
\sum_{X_{\beta}, X_{\alpha_{j-1}} \supset X_{\beta} \supset X_{\alpha_{j+1}}} \bar{F}_{\alpha_{0}, \ldots, \alpha_{j-1}, \beta, \alpha_{j+1}, \ldots, \alpha_{p}=\alpha}
$$

Such an element is determined by $j \in\{1, \ldots, p-1\}$ and an incomplete flag $X_{\alpha_{0}} \supset \ldots \supset X_{\alpha_{j-1}} \supset X_{\alpha_{j+1}} \supset$ $\ldots \supset X_{\alpha_{p}}=X_{\alpha}$ with $l_{\alpha_{i}}=i$.

We denote by $F_{\alpha_{0}, \ldots, \alpha_{p}}$ the image in $\left(\mathcal{F}_{\alpha}\right)_{\mathbb{Z}}$ of the element $\bar{F}_{\alpha_{0}, \ldots, \alpha_{p}}$. For $p=0, \ldots, k$, we set $\left(\mathcal{F}^{p}(\mathcal{C})\right)_{\mathbb{Z}}=\oplus_{X_{\alpha}, l_{\alpha}=p}\left(\mathcal{F}_{\alpha}\right)_{\mathbb{Z}}, \mathcal{F}^{p}(\mathcal{C})=\left(\mathcal{F}^{p}(\mathcal{C})\right)_{\mathbb{Z}} \otimes \mathbb{C}, \mathcal{F}(\mathcal{C})=\oplus_{p=1}^{N} \mathcal{F}^{p}(\mathcal{C})$. We define the differential $d_{\mathbb{Z}}:\left(\mathcal{F}^{p}(\mathcal{C})\right)_{\mathbb{Z}} \rightarrow\left(\mathcal{F}^{p+1}(\mathcal{C})\right)_{\mathbb{Z}}$ by

$$
d_{\mathbb{Z}}: F_{\alpha_{0}, \ldots, \alpha_{p}} \mapsto \sum_{X_{\beta}, X_{\alpha_{p}} \supset X_{\beta}} F_{\alpha_{0}, \ldots, \alpha_{p}, \beta},
$$

$d_{\mathbb{Z}}^{2}=0$. Tensoring $d_{\mathbb{Z}}$ with $\mathbb{C}$, we obtain the differential $d: \mathcal{F}^{p}(\mathcal{C}) \rightarrow \mathcal{F}^{p+1}(\mathcal{C})$. In particular, we have $H^{p}(\mathcal{F}(\mathcal{C}), d)=H^{p}\left((\mathcal{F}(\mathcal{C}))_{\mathbb{Z}}, d_{\mathbb{Z}}\right) \otimes \mathbb{C}$.

We have $H^{p}(\mathcal{F}(\mathcal{C}), d)=0$ for $p \neq k$ and $\operatorname{dim} H^{k}(\mathcal{F}(\mathcal{C}), d)=|\chi(U(\mathcal{C}))|$, where $\chi(U(\mathcal{C}))$ is the Euler characteristic of the complement $U(\mathcal{C})$, see [SV, Corollary 2.8] 


\subsection{Duality}

The vector spaces $\mathcal{A}^{p}(\mathcal{C})$ and $\mathcal{F}^{p}(\mathcal{C})$ are dual, see [SV]. The pairing $\mathcal{A}^{p}(\mathcal{C}) \otimes \mathcal{F}^{p}(\mathcal{C}) \rightarrow \mathbb{C}$ is defined as follows. For $H_{j_{1}}, \ldots, H_{j_{p}}$ in general position, set $F\left(H_{j_{1}}, \ldots, H_{j_{p}}\right)=F_{\alpha_{0}, \ldots, \alpha_{p}}$, where $X_{\alpha_{0}}=\mathbb{C}^{k}$, $X_{\alpha_{1}}=H_{j_{1}}, \ldots, X_{\alpha_{p}}=H_{j_{1}} \cap \cdots \cap H_{j_{p}}$. Then we define $\left\langle\left(H_{j_{1}}, \ldots, H_{j_{p}}\right), F_{\alpha_{0}, \ldots, \alpha_{p}}\right\rangle=(-1)^{|\sigma|}$, if $F_{\alpha_{0}, \ldots, \alpha_{p}}=F\left(H_{j_{\sigma(1)}}, \ldots, H_{j_{\sigma(p)}}\right)$ for some $\sigma \in S_{p}$, and $\left\langle\left(H_{j_{1}}, \ldots, H_{j_{p}}\right), F_{\alpha_{0}, \ldots, \alpha_{p}}\right\rangle=0$ otherwise.

An element $F \in \mathcal{F}^{k}(\mathcal{C})$ is called singular if $F$ annihilates the image of the map $d^{(a)}: \mathcal{A}^{k-1}(\mathcal{C}) \rightarrow$ $\mathcal{A}^{k}(\mathcal{C})$, see [V3]. Denote by $\operatorname{Sing}_{a} \mathcal{F}^{k}(\mathcal{C}) \subset \mathcal{F}^{k}(\mathcal{C})$ the subspace of all singular vectors.

\subsection{Contravariant map and form, see [SV]}

The weights $a$ determines the contravariant map

$$
\mathcal{S}^{(a)}: \mathcal{F}^{p}(\mathcal{C}) \rightarrow \mathcal{A}^{p}(\mathcal{C}), \quad F_{\alpha_{0}, \ldots, \alpha_{p}} \mapsto \sum a_{j_{1}} \cdots a_{j_{p}}\left(H_{j_{1}}, \ldots, H_{j_{p}}\right)
$$

where the sum is taken over all $p$-tuples $\left(H_{j_{1}}, \ldots, H_{j_{p}}\right)$ such that $H_{j_{1}} \supset X_{\alpha_{1}}, \ldots, H_{j_{p}} \supset X_{\alpha_{p}}$. Identifying $\mathcal{A}^{p}(\mathcal{C})$ with $\mathcal{F}^{p}(\mathcal{C})^{*}$, we consider the map as a bilinear form, $S^{(a)}: \mathcal{F}^{p}(\mathcal{C}) \otimes \mathcal{F}^{p}(\mathcal{C}) \rightarrow \mathbb{C}$. The bilinear form is called the contravariant form. The contravariant form is symmetric. The contravariant map (1.3) defines a homomorphism of complexes $\mathcal{S}^{(a)}:(\mathcal{F}(\mathcal{C}), d) \rightarrow\left(\mathcal{A}(\mathcal{C}), d^{(a)}\right)$, see [SV, Lemma 3.2.5].

\subsection{Generic weights}

Theorem 1.7.1 ([SV, Theorem 3.7]). If the weight a is such that none of the dense edges has weight zero, then the contravariant form is nondegenerate. In particular, we have an isomorphism of complexes $\mathcal{S}:(\mathcal{F}(\mathcal{C}), d) \rightarrow\left(\mathcal{A}(\mathcal{C}), d^{(a)}\right)$.

Notice that none of the dense edges has weight zero if all weights are positive.

If the weight $a$ is such that none of the dense edges has weight zero, then the isomorphism of Theorem 1.7.1 and the graded algebra structure on $\mathcal{A}(\mathcal{C})$ induce a graded algebra structure on $\mathcal{F}(\mathcal{C})$. 


\subsection{Differential forms}

For $j \in J$, fix defining equations $f_{j}=0$ for the hyperplanes $H_{j}$, where $f_{j}=b_{j}^{1} t_{1}+\cdots+b_{j}^{k} t_{k}+z_{j}$ with $b_{j}^{i}, z_{j} \in \mathbb{C}$. Consider the logarithmic differential 1-forms $\omega_{j}=d f_{j} / f_{j}$ on $\mathbb{C}^{k}$. Let $\overline{\mathcal{A}}(\mathcal{C})$ be the exterior $\mathbb{C}$-algebra of differential forms generated by 1 and $\omega_{j}, j \in J$. The map $\mathcal{A}(\mathcal{C}) \rightarrow \overline{\mathcal{A}}(\mathcal{C}),\left(H_{j}\right) \mapsto \omega_{j}$, is an isomorphism. We identify $\mathcal{A}(\mathcal{C})$ and $\overline{\mathcal{A}}(\mathcal{C})$.

For $I=\left\{i_{1}, \ldots, i_{k}\right\} \subset J$, denote $d_{I}=d_{i_{1}, \ldots, i_{k}}=\operatorname{det}_{i, l=1}^{k}\left(b_{i_{l}}^{i}\right)$. Then $\omega_{i_{1}} \wedge \cdots \wedge \omega_{i_{k}}=\frac{d_{i_{1}, \ldots, i_{k}}}{f_{i_{1} \ldots f_{i_{k}}}} d t_{1} \wedge$ $\cdots \wedge d t_{k}$.

\subsection{Master function}

The master function of the weighted arrangement $(\mathcal{C}, a)$ is

$$
\Phi_{\mathcal{C}, a}=\sum_{j \in J} a_{j} \log f_{j}
$$

a multivalued function on $U(\mathcal{C})$. Let $C_{\mathcal{C}, a}=\left\{u \in U(\mathcal{C}) \mid \frac{\partial \Phi_{\mathcal{C}, a}}{\partial t_{i}}(u)=0\right.$ for $\left.i=1, \ldots, k\right\}$ be the critical set of $\Phi_{\mathcal{C}, a}$.

\subsection{Isolated critical points}

For generic weight $a \in\left(\mathbb{C}^{\times}\right)^{n}$, all critical points of $\Phi_{\mathcal{C}, a}$ are nondegenerate and the number of critical points equals $|\chi(U(\mathcal{C}))|$, see [V2, OT1, Si].

Consider the projective space $\mathbb{P}^{k}$ compactifying $\mathbb{C}^{k}$. Assign the weight $a_{\infty}=-\sum_{j \in J} a_{j}$ to the hyperplane $H_{\infty}=\mathbb{P}^{k}-\mathbb{C}^{k}$. Denote by $\overline{\mathcal{C}}$ the arrangement $\left(H_{j}\right)_{j \in J \cup \infty}$ in $\mathbb{P}^{k}$. The weighted arrangement $(\mathcal{C}, a)$ is called unbalanced if the weight of any dense edge of $\overline{\mathcal{C}}$ is nonzero, see [V4]. For example, $(\mathcal{C}, a)$ is unbalanced if all weights $\left(a_{j}\right)_{j \in J}$ are positive.

If $(\mathcal{C}, a)$ is unbalanced, then all critical points of $\Phi_{\mathcal{C}, a}$ are isolated and the sum of their Milnor numbers equals $|\chi(U(\mathcal{C}))|$, see [V4, Section 4].

\subsection{Residue}

Let $\mathcal{O}(U(\mathcal{C}))$ be the algebra of regular functions on $U(\mathcal{C})$ and $I_{\mathcal{C}, a}=\left\langle\frac{\partial \Phi_{\mathcal{C}, a}}{\partial t_{i}} \mid i=1, \ldots, k\right\rangle \subset \mathcal{O}(U(\mathcal{C}))$ the ideal generated by first derivatives of $\Phi_{\mathcal{C}, a}$. Let $\mathcal{O}\left(C_{\mathcal{C}, a}\right)=$ 
$\mathcal{O}(U(\mathcal{C})) / I_{\mathcal{C}, a}$ be the algebra of functions on the critical set and [] $: \mathcal{O}(U(\mathcal{C})) \rightarrow \mathcal{O}\left(C_{\mathcal{C}, a}\right), f \mapsto[f]$, the projection. We assume that all critical points are isolated. In that case the algebra $\mathcal{O}\left(C_{\mathcal{C}, a}\right)$ is finite-dimensional and the elements $\left[1 / f_{j}\right], j \in J$, generate $\mathcal{O}\left(C_{\mathcal{C}, a}\right)$ as an algebra, see [V4, Lemma 2.5].

Let $\mathcal{R}: \mathcal{O}\left(C_{\mathcal{C}, a}\right) \rightarrow \mathbb{C}$ be the Grothendieck residue,

$$
[f] \mapsto \frac{1}{(2 \pi i)^{k}} \operatorname{Res} \frac{f}{\prod_{j=1}^{k} \frac{\partial \Phi_{\mathcal{C}, a}}{\partial t_{j}}}=\frac{1}{(2 \pi i)^{k}} \int_{\Gamma} \frac{f d t_{1} \wedge \cdots \wedge d t_{k}}{\prod_{j=1}^{k} \frac{\partial \Phi_{\mathcal{C}, a}}{\partial t_{j}}}
$$

Here $\Gamma$ is the real $k$-cycle defined by the equations $\left|\frac{\partial \Phi_{\mathcal{C}, a}}{\partial t_{j}}\right|=\epsilon_{j}, j=1, \ldots, k$, where $\epsilon_{j}$ are small positive numbers, see $[\mathrm{GH}]$. Define the residue bilinear form $(,)_{C_{\mathcal{C}, a}}$ on $\mathcal{O}\left(C_{\mathcal{C}, a}\right)$ by $([f],[g])_{C_{\mathcal{C}, a}}=\mathcal{R}([f][g])$. This form is nondegenerate, see $[\mathrm{AGV}]$, and $([f][g],[h])_{C_{\mathcal{C}, a}}=([f],[g][h])_{C_{\mathcal{C}, a}}$ for all $[f],[g],[h] \in \mathcal{O}\left(C_{\mathcal{C}, a}\right)$, thus $\left(\mathcal{O}\left(C_{\mathcal{C}, a}\right),(,)_{C_{\mathcal{C}, a}}\right)$ is a Frobenius algebra.

\subsection{Orthogonal projection}

Let $\pi^{\perp}: \mathcal{F}^{k}(\mathcal{C}) \rightarrow \operatorname{Sing}_{a} \mathcal{F}^{k}(\mathcal{C})$ be the orthogonal projection with respect to $S^{(a)}$.

If the weight $a \in\left(\mathbb{C}^{\times}\right)^{n}$ is unbalanced, then $d \mathcal{F}^{k-1}(\mathcal{C})=\operatorname{Sing}_{a} \mathcal{F}^{k}(\mathcal{C})^{\perp}$, where $d \mathcal{F}^{k-1}(\mathcal{C}) \subset \mathcal{F}^{k}(\mathcal{C})$ is the image of the differential defined by (1.2) and $\operatorname{Sing}_{a} \mathcal{F}^{k}(\mathcal{C})^{\perp} \subset \mathcal{F}^{k}(\mathcal{C})$ is the orthogonal complement to $\operatorname{Sing}_{a} \mathcal{F}^{k}(\mathcal{C})$ with respect to $S^{(a)}$, see [V6, Lemma 2.14].

Define the map

$$
\nu_{\mathcal{C}}: \mathcal{F}^{k}(\mathcal{C}) \rightarrow \mathcal{O}\left(C_{\mathcal{C}, a}\right), \quad F \mapsto[f]
$$

where $f$ is defined by the formula $\mathcal{S}^{(a)}(F)=f d t_{1} \wedge \cdots \wedge d t_{k}$. Clearly, $\nu_{\mathcal{C}}\left(\operatorname{Sing}_{a} \mathcal{F}^{k}(\mathcal{C})^{\perp}\right)=\nu_{\mathcal{C}}\left(d \mathcal{F}^{k-1}(\mathcal{C})\right)=$ 0 , since $\omega^{(a)}=0$ on $C_{\mathcal{C}, a}$.

Theorem 1.12.1 ([V6]). If the weight $a \in\left(\mathbb{C}^{\times}\right)^{n}$ is unbalanced, then the map $\left.\nu_{\mathcal{C}}\right|_{\operatorname{Sing}_{a} \mathcal{F}^{k}(\mathcal{C})}: \operatorname{Sing}_{a} \mathcal{F}^{k}(\mathcal{C}) \rightarrow$ $\mathcal{O}\left(C_{\mathcal{C}, a}\right)$ is an isomorphism of vector spaces. The isomorphism $\nu_{\mathcal{C}}$ identifies the residue form on $\mathcal{O}\left(C_{\mathcal{C}, a}\right)$ and the contravariant form on Sing $\mathcal{F}^{k}(\mathcal{C})$ multiplied by $(-1)^{k}, S^{(a)}(f, g)=(-1)^{k}\left(\nu_{\mathcal{C}}(f), \nu_{\mathcal{C}}(g)\right)_{C_{\mathcal{C}, a}}$ for $f, g \in \operatorname{Sing}_{a} \mathcal{F}^{k}(\mathcal{C})$.

Remark 1. If the weight $a \in\left(\mathbb{C}^{\times}\right)^{n}$ is unbalanced, then the isomorphism $\mu_{\mathcal{C}}$ induces a commutative associative algebra structure on $\operatorname{Sing}_{a} \mathcal{F}^{k}(\mathcal{C})$. Together with the contravariant form $\left.S^{(a)}\right|_{\text {Sing }_{a} \mathcal{F}^{k}}$ it is a 
Frobenius algebra. The algebra of multiplication operators on $\operatorname{Sing}_{a} \mathcal{F}^{k}(\mathcal{C})$ is called the Bethe algebra of the weighted arrangement $(\mathcal{C}, a)$. This Bethe algebra is an analog of the Bethe algebra in the theory of quantum integrable models, see, for example, [MTV1, MTV2, V3, V4].

\subsection{Integral structure on $\mathcal{O}\left(C_{\mathcal{C}, a}\right)$ and $\operatorname{Sing}_{a} \mathcal{F}^{k}(\mathcal{C})$}

If the weight $a$ is unbalanced, the formula $H^{p}(\mathcal{F}(\mathcal{C}), d)=H^{p}\left((\mathcal{F}(\mathcal{C}))_{\mathbb{Z}}, d_{\mathbb{Z}}\right) \otimes \mathbb{C}$ and the isomorphism $\left.\nu_{\mathcal{C}}\right|_{\operatorname{Sing}_{a} \mathcal{F}^{k}(\mathcal{C})}: H^{k}(\mathcal{F}(\mathcal{C}), d) \cong \operatorname{Sing}_{a} \mathcal{F}(\mathcal{C}) \rightarrow \mathcal{O}\left(C_{\mathcal{C}, a}\right)$ define an integral structure on $\mathcal{O}\left(C_{\mathcal{C}, a}\right)$. More precisely, for a $k$-flag of edges $X_{\alpha_{0}} \supset X_{\alpha_{1}} \supset \cdots \supset X_{\alpha_{k}}$, let $\mathcal{S}^{(a)}\left(F_{\alpha_{0}, \ldots, \alpha_{k}}\right)=f_{\alpha_{0}, \ldots, \alpha_{k}} d t_{1} \wedge \cdots \wedge d t_{k}$. Denote by $w_{\alpha_{0}, \ldots, \alpha_{k}}$ the element $\left[f_{\alpha_{0}, \ldots, \alpha_{k}}\right] \in \mathcal{O}\left(C_{\mathcal{C}, a}\right)$.

Corollary 1.13.1 ([V6]). If the weight a is unbalanced, then the set of all elements $\left\{w_{\alpha_{0}, \ldots, \alpha_{k}}\right\}$, labeled by all $k$-flag of edges of $\mathcal{C}$, spans the vector space $\mathcal{O}\left(C_{\mathcal{C}, a}\right)$. All linear relations between the elements of the set are corollaries of the relations

$$
\begin{aligned}
& \sum_{X_{\beta}, X_{\alpha_{j-1}} \supset X_{\beta} \supset X_{\alpha_{j+1}}} w_{\alpha_{0}, \ldots, \alpha_{j-1}, \beta, \alpha_{j+1}, \ldots, \alpha_{p}=\alpha}=0, \\
& \sum_{X_{\beta}, X_{\alpha_{p}} \supset X_{\beta}} w_{\alpha_{0}, \ldots, \alpha_{p}, \beta}=0
\end{aligned}
$$

cf. formulas (1.1), (1.2).

Similarly, for a $k$-flag of edges $X_{\alpha_{0}} \supset X_{\alpha_{1}} \supset \cdots \supset X_{\alpha_{k}}$, let $v_{\alpha_{0}, \ldots, \alpha_{k}}$ be the orthogonal projection of $F_{\alpha_{0}, \ldots, \alpha_{k}}$ to $\operatorname{Sing}_{a} \mathcal{F}^{k}(\mathcal{C})$.

Corollary 1.13.2 ([V6]). If the weight a is unbalanced, then the set of all elements $\left\{v_{\alpha_{0}, \ldots, \alpha_{k}}\right\}$, labeled by all $k$-flag of edges of $\mathcal{C}$, spans the vector space $\operatorname{Sing}_{a} \mathcal{F}^{k}(\mathcal{C})$. All linear relations between the elements of the set are corollaries of the relations

$$
\begin{aligned}
& \sum_{X_{\beta}, X_{\alpha_{j-1}} \supset X_{\beta} \supset X_{\alpha_{j+1}}} v_{\alpha_{0}, \ldots, \alpha_{j-1}, \beta, \alpha_{j+1}, \ldots, \alpha_{p}=\alpha}=0, \\
& \sum_{X_{\beta}, X_{\alpha_{p}} \supset X_{\beta}} v_{\alpha_{0}, \ldots, \alpha_{p}, \beta}=0,
\end{aligned}
$$

cf. formulas (1.1), (1.2).

We have $\nu_{\mathcal{C}}: v_{\alpha_{0}, \ldots, \alpha_{k}} \mapsto w_{\alpha_{0}, \ldots, \alpha_{k}}$. The elements $\left\{w_{\alpha_{0}, \ldots, \alpha_{k}}\right\} \subset \mathcal{O}\left(C_{\mathcal{C}, a}\right)$ and $\left\{v_{\alpha_{0}, \ldots, \alpha_{k}}\right\} \subset$ $\operatorname{Sing}_{a} \mathcal{F}^{k}(\mathcal{C})$ are called the marked elements. The relations (1.6), (1.7) are called the marked relations. 


\subsection{Combinatorial connection, I}

Consider a deformation $\mathcal{C}(s)$ of the arrangement $\mathcal{C}$, which preserves the combinatorics of $\mathcal{C}$. Assume that the edges of $\mathcal{C}(s)$ can be identified with the edges of $\mathcal{C}$ so that the elements in formula (1.1) and the differential in formula (1.2) do not depend on $s$. Then for every $s$, the elements $\left\{w_{\alpha_{0}, \ldots, \alpha_{k}}(s)\right\}$ span $\mathcal{O}\left(C_{\mathcal{C}(s), a}\right)$ as a vector space with linear relations (1.6) not depending on $s$. This allows us to identify all the vector spaces $\mathcal{O}\left(C_{\mathcal{C}(s), a}\right)$. In particular, if an element $w(s) \in \mathcal{O}\left(C_{\mathcal{C}(s), a}\right)$ is given, then the derivative $\frac{d w}{d s}$ is well-defined. This construction is called the combinatorial connection on the family of algebras $\mathcal{O}\left(C_{\mathcal{C}(s), a}\right)$, see [V5]. All the elements $\left\{w_{\alpha_{0}, \ldots, \alpha_{k}}(s)\right\}$ are flat sections of the combinatorial connection.

Similarly we can define the combinatorial connection on the family of vector spaces $\operatorname{Sing}_{a} \mathcal{F}^{k}(\mathcal{C}(s))$.

\subsection{5}

Arrangement with normal crossings

An essential arrangement $\mathcal{C}$ is with normal crossings, if exactly $k$ hyperplanes meet at every vertex of $\mathcal{C}$. Assume that $\mathcal{C}$ is an essential arrangement with normal crossings.

A basis of $\mathcal{A}^{p}(\mathcal{C})$ is formed by $\left(H_{j_{1}}, \ldots, H_{j_{p}}\right)$, where $\left\{j_{1}<\cdots<j_{p}\right\}$ are independent ordered $p$ element subsets of $J$. The dual basis of $\mathcal{F}^{p}(\mathcal{C})$ is formed by the corresponding vectors $F\left(H_{j_{1}}, \ldots, H_{j_{p}}\right)$. These bases of $\mathcal{A}^{p}(\mathcal{C})$ and $\mathcal{F}^{p}(\mathcal{C})$ are called standard. We have

$$
F\left(H_{j_{1}}, \ldots, H_{j_{p}}\right)=(-1)^{|\sigma|} F\left(H_{j_{\sigma(1)}}, \ldots, H_{j_{\sigma(p)}}\right), \quad \text { for } \sigma \in \Sigma_{p}
$$

For an independent subset $\left\{j_{1}, \ldots, j_{p}\right\}$, we have $S^{(a)}\left(F\left(H_{j_{1}}, \ldots, H_{j_{p}}\right), F\left(H_{j_{1}}, \ldots, H_{j_{p}}\right)\right)=a_{j_{1}} \cdots a_{j_{p}}$ and $S^{(a)}\left(F\left(H_{j_{1}}, \ldots, H_{j_{p}}\right), F\left(H_{i_{1}}, \ldots, H_{i_{k}}\right)\right)=0$ for distinct elements of the standard basis. If $a$ is unbalanced, then the marked elements in $\mathcal{O}\left(C_{\mathcal{C}, a}\right)$ are

$$
w_{i_{1}, \ldots, i_{k}}=d_{i_{1}, \ldots, i_{k}} \frac{a_{i_{1}}}{\left[f_{i_{1}}\right]} \ldots \frac{a_{i_{k}}}{\left[f_{i_{k}}\right]}
$$

where $\left\{i_{1}, \ldots, i_{k}\right\}$ runs through the set of all independent $k$-element subsets of $J$. We have $w_{i_{\sigma(1)}, \ldots, i_{\sigma(k)}}=$ $(-1)^{\sigma} w_{i_{1}, \ldots, i_{k}}$ for $\sigma \in \Sigma_{k}$. We put $w_{i_{1}, \ldots, i_{k}}=0$ if the set $\left\{i_{1}, \ldots, i_{k}\right\}$ is dependent. The marked relations are labeled by independent subsets $\left\{i_{2}, \ldots, i_{k}\right\}$ and have the form $\sum_{j \in J} w_{j, i_{2}, \ldots, i_{k}}=0$. The marked 
elements $v_{i_{1}, \ldots, i_{k}}$ in $\operatorname{Sing}_{a} \mathcal{F}^{k}(\mathcal{C})$ are orthogonal projections to $\operatorname{Sing}_{a} \mathcal{F}^{k}(\mathcal{C})$ of the elements $F\left(H_{i_{1}}, \ldots, H_{i_{k}}\right)$ with the skew-symmetry property $v_{i_{\sigma(1)}, \ldots, i_{\sigma(k)}}=(-1)^{\sigma} v_{i_{1}, \ldots, i_{k}}$ for $\sigma \in \Sigma_{k}$ and the marked relations $\sum_{j \in J} v_{j, i_{2}, \ldots, i_{k}}=0$ labeled by independent subsets $\left\{i_{2}, \ldots, i_{k}\right\}$.

For any independent ordered subset $j_{1}, \ldots, j_{p} \in J$ we denote $F_{j_{1}, \ldots, j_{p}}=F\left(H_{j_{1}}, \ldots, H_{j_{p}}\right) \in \mathcal{F}^{p}(\mathcal{C})$ and set $F_{j_{1}, \ldots, j_{p}}=0$ if $j_{1}, \ldots, j_{p}$ is a dependent subset.

Corollary 1.15.1. The orthogonal complement $\operatorname{Sing}_{a} \mathcal{F}^{k}(\mathcal{C})$ is generated by the elements $\sum_{j \in J} F_{j, i_{1}, \ldots, i_{k-1}}$ labeled by independent subsets $\left\{i_{1}, \ldots, i_{k-1}\right\} \in J$, and an element of $\mathcal{F}^{k}(\mathcal{C})$ lies in Sing $\mathcal{F}^{k}(\mathcal{C})$ if and only if it is orthogonal to all the elements $\sum_{j \in J} F_{j, i_{1}, \ldots, i_{k-1}}$. 


\section{CHAPTER 2}

\section{Family of parallelly transported hyperplanes}

\subsection{Arrangement in $\mathbb{C}^{n} \times \mathbb{C}^{k}$}

Consider $\mathbb{C}^{k}$ with coordinates $t_{1}, \ldots, t_{k}, \mathbb{C}^{n}$ with coordinates $z_{1}, \ldots, z_{n}$, the projection $\tau: \mathbb{C}^{n} \times \mathbb{C}^{k} \rightarrow \mathbb{C}^{n}$. Fix $n$ nonzero linear functions on $\mathbb{C}^{k}, g_{j}=b_{j}^{1} t_{1}+\cdots+b_{j}^{k} t_{k}, j \in J$, where $b_{j}^{i} \in \mathbb{C}$. Assume that the functions $\left\{g_{j}\right\}_{j \in J}$, span the dual space $\left(\mathbb{C}^{k}\right)^{*}$.

Define $n$ linear functions on $\mathbb{C}^{n} \times \mathbb{C}^{k}, f_{j}=g_{j}+z_{j}, j \in J$. Consider the arrangement of hyperplanes $\mathcal{C}=\left\{H_{j}\right\}_{j \in J}$ in $\mathbb{C}^{n} \times \mathbb{C}^{k}$, where $H_{j}$ is the zero set of $f_{j}$, and denote by $U(\mathcal{C})=\mathbb{C}^{n} \times \mathbb{C}^{k}-\cup_{j \in J} H_{j}$ the complement. For every $x=\in \mathbb{C}^{n}$, the arrangement $\mathcal{C}$ induces an arrangement $\mathcal{C}(x)$ in the fiber $\tau^{-1}(x) \cong \mathbb{C}^{k}$. Then $\mathcal{C}(x)$ consists of hyperplanes $\left\{H_{j}(x)\right\}_{j \in J}$, defined in $\mathbb{C}^{k}$ by the equations $g_{j}+x_{j}=0$. Thus $\{\mathcal{C}(x)\}_{x \in \mathbb{C}^{n}}$ is a family of arrangements in $\mathbb{C}^{k}$, whose hyperplanes are transported parallelly to themselves as $x$ changes. Denote by $U(\mathcal{C}(x))=\mathbb{C}^{k}-\cup_{j \in J} H_{j}(x)$ the complement. For almost all $x \in \mathbb{C}^{k}$ the arrangement $\mathcal{C}(x)$ is with normal crossings. The subset $\Delta \subset \mathbb{C}^{n}$ where this does not hold, is a hypersurface called the discriminant. On the discriminant see, for example, [BB, V4].

\subsection{Combinatorial connection, II}

For any $x^{1}, x^{2} \in \mathbb{C}^{n}-\Delta$, the spaces $\mathcal{F}^{p}\left(\mathcal{C}\left(x^{1}\right)\right), \mathcal{F}^{p}\left(\mathcal{C}\left(x^{2}\right)\right)$ are canonically identified if a vector $F\left(H_{j_{1}}\left(x^{1}\right), \ldots, H_{j_{p}}\left(x^{1}\right)\right)$ of the first space is identified with the vector $F\left(H_{j_{1}}\left(x^{2}\right), \ldots, H_{j_{p}}\left(x^{2}\right)\right)$ of the second, in other words, if we identify the standard bases of these spaces.

Assume that a weight $a \in\left(\mathbb{C}^{\times}\right)^{n}$ is given. Then each arrangement $\mathcal{C}(x)$ is weighted. The identification of spaces $\mathcal{F}^{p}\left(\mathcal{C}\left(x^{1}\right)\right), \mathcal{F}^{p}\left(\mathcal{C}\left(x^{2}\right)\right)$ for $x^{1}, x^{2} \in \mathbb{C}^{n}-\Delta$ identifies the corresponding subspaces $\operatorname{Sing}_{a} \mathcal{F}^{k}\left(\mathcal{C}\left(x^{1}\right)\right)$, $\operatorname{Sing}_{a} \mathcal{F}^{k}\left(\mathcal{C}\left(x^{2}\right)\right)$ and contravariant forms.

Assume that the weighted arrangement $(\mathcal{C}(x), a)$ is unbalanced for some $x \in \mathbb{C}^{n}-\Delta$, then $(\mathcal{C}(x), a)$ is unbalanced for all $x \in \mathbb{C}^{n}-\Delta$. The identification of $\operatorname{Sing}_{a} \mathcal{F}^{k}\left(\mathcal{C}\left(x^{1}\right)\right)$ and $\operatorname{Sing}_{a} \mathcal{F}^{k}\left(\mathcal{C}\left(x^{2}\right)\right)$ also identifies the marked elements $v_{j_{1}, \ldots, j_{k}}\left(x^{1}\right)$ and $v_{j_{1}, \ldots, j_{k}}\left(x^{2}\right)$, see Section 1.15. For $x \in \mathbb{C}^{n}-\Delta$, denote $V=\mathcal{F}^{k}(\mathcal{C}(x))$, 
$\operatorname{Sing}_{a} V=\operatorname{Sing}_{a} \mathcal{F}^{k}(\mathcal{C}(x)), v_{j_{1}, \ldots, j_{k}}=v_{j_{1}, \ldots, j_{k}}(x)$. The triple $\left(V, \operatorname{Sing}_{a} V, S^{(a)}\right)$, with marked elements $v_{j_{1}, \ldots, j_{k}}$, does not depend on $x$ under the identification.

As a result of this reasoning we obtain the canonically trivialized vector bundle

$$
\sqcup_{x \in \mathbb{C}^{n}-\Delta} \mathcal{F}^{k}(\mathcal{C}(x)) \rightarrow \mathbb{C}^{n}-\Delta
$$

with the canonically trivialized subbundle $\sqcup_{x \in \mathbb{C}^{n}-\Delta} \operatorname{Sing}_{a} \mathcal{F}^{k}(\mathcal{C}(x)) \rightarrow \mathbb{C}^{n}-\Delta$ and the constant contravariant form on the fibers. This trivialization identifies the bundle in (2.1) with the bundle $\left(\mathbb{C}^{n}-\Delta\right) \times V \rightarrow$ $\mathbb{C}^{n}-\Delta$ and identifies the subbundle $\sqcup_{x \in \mathbb{C}^{n-\Delta}} \operatorname{Sing}_{a} \mathcal{F}^{k}(\mathcal{C}(x)) \rightarrow \mathbb{C}^{n}-\Delta$ with the subbbundle

$$
\left(\mathbb{C}^{n}-\Delta\right) \times\left(\operatorname{Sing}_{a} V\right) \rightarrow \mathbb{C}^{n}-\Delta
$$

The bundle in (2.2) is called the combinatorial bundle, the flat connection on it is called combinatorial, see Section 1.14 and [V4, V5].

\subsection{Gauss-Manin connection on $\left(\mathbb{C}^{n}-\Delta\right) \times\left(\operatorname{Sing}_{a} V\right) \rightarrow \mathbb{C}^{n}-\Delta$}

The master function is $\Phi_{\mathcal{C}, a}=\sum_{j \in J} a_{j} \log f_{j}$, a multivalued function on $U(\mathcal{C})$. Let $\kappa \in \mathbb{C}^{\times}$. The function $e^{\Phi_{\mathcal{C}, a} / \kappa}$ defines a rank one local system $\mathcal{L}_{\kappa}$ on $U(\mathcal{C})$ whose horizontal sections over open subsets of $U(\mathcal{C})$ are univalued branches of $e^{\Phi_{\mathcal{C}, a} / \kappa}$ multiplied by complex numbers, see, for example, [SV, V2]. The vector bundle

$$
\sqcup_{x \in \mathbb{C}^{n}-\Delta} H_{k}\left(U(\mathcal{C}(x)),\left.\mathcal{L}_{\kappa}\right|_{U(\mathcal{C}(x))}\right) \rightarrow \mathbb{C}^{n}-\Delta
$$

is called the homology bundle. The homology bundle has a canonical flat Gauss-Manin connection.

For a fixed $x \in \mathbb{C}^{n}-\Delta$, choose $\gamma \in H_{k}\left(U(\mathcal{C}(x)),\left.\mathcal{L}_{\kappa}\right|_{U(\mathcal{C}(x))}\right)$. The linear map $\{\gamma\}: \mathcal{A}^{k}(\mathcal{C}(x)) \rightarrow \mathbb{C}$, $\omega \mapsto \int_{\gamma} e^{\Phi_{\mathcal{C}, a} / \kappa} \omega$, is an element of $\operatorname{Sing}_{a} \mathcal{F}^{k}(\mathcal{C}(x))$ by Stokes' theorem. It is known that for generic $\kappa$ any element of $\operatorname{Sing}_{a} \mathcal{F}^{k}(\mathcal{C}(x))$ corresponds to a certain $\gamma$ and in that case this construction gives the integration isomorphism

$$
H_{k}\left(U(\mathcal{C}(x)),\left.\mathcal{L}_{\kappa}\right|_{U(\mathcal{C}(x))}\right) \rightarrow \operatorname{Sing}_{a} \mathcal{F}^{k}(\mathcal{C}(x))
$$


see [SV]. The precise values of $\kappa$, such that (2.3) is an isomorphism, can be deduced from the determinant formula in [V1].

For generic $\kappa$ the fiber isomorphisms (2.3) define an isomorphism of the homology bundle and the combinatorial bundle (2.2). The Gauss-Manin connection induces a connection on the combinatorial bundle. That connection on the combinatorial bundle is also called the Gauss-Manin connection.

Thus, there are two connections on the combinatorial bundle: the combinatorial connection and the GaussManin connection depending on $\kappa$. In this situation we consider the differential equations for flat sections of the Gauss-Manin connection with respect to the combinatorially flat standard basis. Namely, let $\gamma(x) \in$ $H_{k}\left(U(\mathcal{C}(x)),\left.\mathcal{L}_{\kappa}\right|_{U(\mathcal{C}(x))}\right)$ be a flat section of the Gauss-Manin connection. Let us write the corresponding section $I_{\gamma}(x)$ of the bundle $\left(\mathbb{C}^{n}-\Delta\right) \times \operatorname{Sing}_{a} V \rightarrow \mathbb{C}^{n}-\Delta$ in the combinatorially flat standard basis, $I_{\gamma}(x)=\sum \underset{\substack{\text { independent } \\\left\{j_{1}<\cdots<j_{k}\right\} \subset J}}{j_{\gamma}, \ldots, j_{k}}(x) F\left(H_{j_{1}}, \ldots, H_{j_{k}}\right), I_{\gamma}^{j_{1}, \ldots, j_{k}}(x)=\int_{\gamma(x)} e^{\Phi_{\mathcal{C}, a} / \kappa} \omega_{j_{1}} \wedge \cdots \wedge \omega_{j_{k}}$. We may rewrite it as $I_{\gamma}(x)=\sum \underset{\substack{\text { independent } \\\left\{j_{1}<\cdots<j_{k}\right\} \subset J}}{ } I_{\gamma}^{j_{1}, \ldots, j_{k}}(x) v_{j_{1}, \ldots, j_{k}}$ since $I_{\gamma}(x) \in \operatorname{Sing}_{a} V$. For $I=\sum I^{j_{1}, \ldots, j_{k}} v_{j_{1}, \ldots, j_{k}}$ and $j \in J$, we denote $\frac{\partial I}{\partial z_{j}}=\sum \frac{\partial I^{j_{1}, \ldots, j_{k}}}{\partial z_{j}} v_{j_{1}, \ldots, j_{k}}$. This formula defines the combinatorial connection on the combinatorial bundle.

The section $I_{\gamma}$ satisfies the Gauss-Manin differential equations

$$
\kappa \frac{\partial I}{\partial z_{j}}(x)=K_{j}(x) I(x), \quad j \in J
$$

where $K_{j}(x) \in \operatorname{End}\left(\operatorname{Sing}_{a} V\right)$. See a description of the operators $K_{j}(x)$, for example, in [OT2, V2, V4].

\subsection{Critical set}

Denote by $C_{\mathcal{C}, a}$ the critical set of $\Phi_{\mathcal{C}, a}$ in the $\mathbb{C}^{k}$-direction,

$$
C_{\mathcal{C}, a}=\left\{(x, u) \in U(\mathcal{C}) \subset \mathbb{C}^{n} \times \mathbb{C}^{k} \mid \frac{\partial \Phi_{\mathcal{C}, a}}{\partial t_{i}}(x, u)=0 \text { for } i=1, \ldots, k\right\}
$$

Let $\mathcal{O}\left(C_{\mathcal{C}(x), a}\right)$ be the algebra of regular functions on $C_{\mathcal{C}(x), a}=C_{\mathcal{C}, a} \cap \tau^{-1}(x)$. Namely, for $x \in$ $\mathbb{C}^{n}$, let $I_{\mathcal{C}(x), a}$ be the ideal in $\mathcal{O}(U(\mathcal{C}(x)))$ generated by $\frac{\partial \Phi_{\mathcal{C}, a}}{\partial t_{i}}, i=1, \ldots, k$. We set $\mathcal{O}\left(C_{\mathcal{C}(x), a}\right)=$ $\mathcal{O}(U(\mathcal{C}(x))) / I_{\mathcal{C}(x), a}$. Assume that the weight $a$ is such that the pair $(\mathcal{C}(x), a)$ is unbalanced for some $x \in \mathbb{C}^{n}-\Delta$. Then we obtain the vector bundle of algebras $\sqcup_{x \in \mathbb{C}^{n}-\Delta} \mathcal{O}\left(C_{\mathcal{C}(x), a}\right) \rightarrow \mathbb{C}^{n}-\Delta$. For 
$x \in \mathbb{C}^{n}-\Delta$, recall the isomorphism

$$
\nu(x):=\left.\nu_{\mathcal{C}(x)}\right|_{\operatorname{Sing}_{a} \mathcal{F}^{k}(\mathcal{C}(x)}: \operatorname{Sing}_{a} \mathcal{F}^{k}\left(\mathcal{C}(x) \rightarrow \mathcal{O}\left(C_{\mathcal{C}(x), a}\right)\right.
$$

of Theorem 1.12.1. This 'fiber' isomorphism establishes an isomorphism of the bundle $\sqcup_{x \in \mathbb{C}^{n}-\Delta} \mathcal{O}\left(C_{\mathcal{C}(x), a}\right) \rightarrow \mathbb{C}^{n}-\Delta$ and the bundle $\left(\mathbb{C}^{n}-\Delta\right) \times\left(\operatorname{Sing}_{a} V\right) \rightarrow \mathbb{C}^{n}-\Delta$. This isomorphism together with the combinatorial and Gauss-Manin connections on the bundle $\left(\mathbb{C}^{n}-\Delta\right) \times\left(\operatorname{Sing}_{a} V\right) \rightarrow$ $\mathbb{C}^{n}-\Delta$ induces two connections on the bundle of algebras $\sqcup_{x \in \mathbb{C}^{n}-\Delta} \mathcal{O}\left(C_{\mathcal{C}(x), a}\right) \rightarrow \mathbb{C}^{n}-\Delta$, which also are called the combinatorial and Gauss-Manin connections, respectively.

Theorem 2.4.1 ([V4]). If the pair $(\mathcal{C}(x), a)$ is unbalanced for $x \in \mathbb{C}^{n}-\Delta$, then for all $j \in J$, we have

$$
\left(\left[\frac{a_{j}}{f_{j}}\right] *_{x}\right) \circ \nu(x)=\nu(x) \circ K_{j}(x)
$$

where $\left[\frac{a_{j}}{f_{j}}\right] *_{x}$ is the operator of multiplication by $\left[\frac{a_{j}}{f_{j}}\right]$ on $\mathcal{O}\left(C_{\mathcal{C}(x), a}\right)$ and $K_{j}(x) \in \operatorname{End}\left(\operatorname{Sing}{ }_{a} V\right)$ is the operator defined in (2.4).

Remark 2. Recall that $a_{j} / f_{j}=\partial \Phi_{\mathcal{C}, a} / \partial z_{j}$ and the elements $\left[a_{j} / f_{j}\right], j \in J$, generate the algebra $\mathcal{O}\left(C_{\mathcal{C}(x), a}\right)$. Theorem 2.4.1 says that under the isomorphism $\nu(x)$ the operators of multiplication $\left[a_{j} / f_{j}\right] *_{x}$ on $\mathcal{O}\left(C_{\mathcal{C}(x), a}\right)$ are identified with the operators $K_{j}(x)$ in the Gauss-Manin differential equations (2.4). The correspondence of Theorem 2.4.1 defines a commutative algebra structure on Sing ${ }_{a} V$, the structure depending on $x$. The multiplication in this commutative algebra is generated by the operators $K_{j}(x), j \in J$. The correspondence of Theorem 2.4.1 also defines the Gauss-Manin differential equations on the bundle of algebras in terms of the multiplication in the fiber algebras, see these differential equations in [V6, Theorem 3.9].

\subsection{Formulas for multiplication}

Recall that for $j \in J$, we denote $p_{j}=\left[\frac{\partial \Phi}{\partial z_{j}}\right]=\left[\frac{a_{j}}{f_{j}}\right] \in \mathcal{O}\left(C_{\mathcal{C}(x), a}\right)$. Then $w_{i_{1}, \ldots, i_{k}}=d_{i_{1}, \ldots, i_{k}} p_{i_{1}} \ldots p_{i_{k}}$, and for any $i_{1}, \ldots, i_{k-1} \in J$ we have

$$
\sum_{j \in J} d_{j, i_{1}, \ldots, i_{k-1}} p_{j}=0
$$


For a subset $I=\left\{i_{1}, \ldots, i_{k+1}\right\} \subset J$ denote

$$
f_{I}(z)=\sum_{j=1}^{k+1}(-1)^{j+1} z_{i_{j}} d_{i_{1}, \ldots, \widehat{i_{j}}, \ldots, i_{k+1}}
$$

Lemma 2.5.1. We have

$$
f_{I}(z) \prod_{j=1}^{k+1} \frac{a_{i_{j}}}{f_{i_{j}}}=\sum_{j=1}^{k+1}(-1)^{j+1} a_{i_{j}} d_{i_{1}, \ldots, \hat{i_{j}}, \ldots, i_{k+1}} \prod_{m=1, m \neq j}^{k+1} \frac{a_{i_{m}}}{f_{i_{m}}}
$$

Proof. We have $\sum_{j=1}^{k+1}(-1)^{j+1}\left(f_{i_{j}}-z_{i_{j}}\right) d_{i_{1}, \ldots, \hat{i_{j}}, \ldots, i_{k+1}}=0$. That implies the lemma.

Corollary 2.5.2. Assume that a subset $\left\{i_{1}, \ldots, i_{k+1}\right\} \subset J$ consists of distinct elements and contains a $k$-element independent subset. Then we have an identity in $\mathcal{O}\left(C_{\mathcal{C}(x), a}\right)$ :

$$
f_{i_{1}, \ldots, i_{k+1}}(z) p_{i_{1}} \ldots p_{i_{k+1}}=\sum_{j=1}^{k+1}(-1)^{j+1} a_{i_{j}} w_{i_{1}, \ldots, \hat{i_{j}}, \ldots, i_{k+1}} .
$$

Proof. The corollary follows from Lemma 2.5.1. 


\section{CHAPTER 3}

\section{Elementary arrangements}

\subsection{Definition}

Consider a $k$-dimensional vector space $X$ with coordinates $t_{1}, t_{2}, \ldots, t_{k}$. Let $\lambda=\left(\lambda_{1}, \lambda_{2}, \ldots, \lambda_{m}\right)$ be a collection of positive integers such that $\sum_{i=1}^{m} \lambda_{i}=k$. Assume that we have a collection of non-intersecting index sets $J_{h}, h=1, \ldots, m$, each with $\left|J_{h}\right|=\lambda_{h}+1$ elements. Denote $J_{\lambda}:=\cup_{h=1}^{m} J_{h}, \lambda^{h}=\lambda_{1}+\cdots+\lambda_{h}$, $\lambda^{0}=0$.

Let $\mathcal{C}_{J_{\lambda}}=\left\{H_{i}\right\}_{i \in J_{\lambda}}$ be a weighted arrangement of affine hyperplanes in $X$ with normal crossings. Each hyperplane $H_{i}$ has weight $a_{i}$ and is defined by an equation $g_{i}\left(t_{1}, \ldots, t_{k}\right)+z_{i}=0$, where $g_{i}=\sum_{l=1}^{k} b_{i}^{l} t_{l}$ is an element of the dual space $X^{*}$ and $z_{i} \in \mathbb{C}$.

Define a subspace $X_{h}^{*}\left(J_{\lambda}\right)=\operatorname{span}\left\{g_{i}\right\}_{i \in \bigcup_{j=1}^{h} J_{j}}$ of $X^{*}$. We have a filtration

$$
X_{1}^{*}\left(J_{\lambda}\right) \subset X_{2}^{*}\left(J_{\lambda}\right) \subset \cdots \subset X_{m}^{*}\left(J_{\lambda}\right) \subset X^{*}
$$

The arrangement $\mathcal{C}_{J_{\lambda}}$ is called elementary of type $\lambda$ if

(i) For any $h \in\{1, \ldots, m\}$ we have $\operatorname{dim} X_{h}^{*}\left(J_{\lambda}\right)=\lambda^{h}$.

(ii) For any $h \in\{1, \ldots, m\}$, any $j \in J_{h}$ and $A:=\left\{\cup_{i=1}^{h} J_{i}\right\}-\{j\}$, we have

$$
\operatorname{dim}\left(\operatorname{span}\left\{g_{l}\right\}_{l \in A}\right)=\lambda^{h} .
$$

In Figure 2 the first elementary arrangement is of type $\lambda=(2)$ and the second is of type $\lambda=(1,1)$ with $J_{1}=\{1,2\}, J_{2}=\{3,4\}$. In this section we always assume that $\mathcal{C}_{J_{\lambda}}$ is an elementary arrangement.
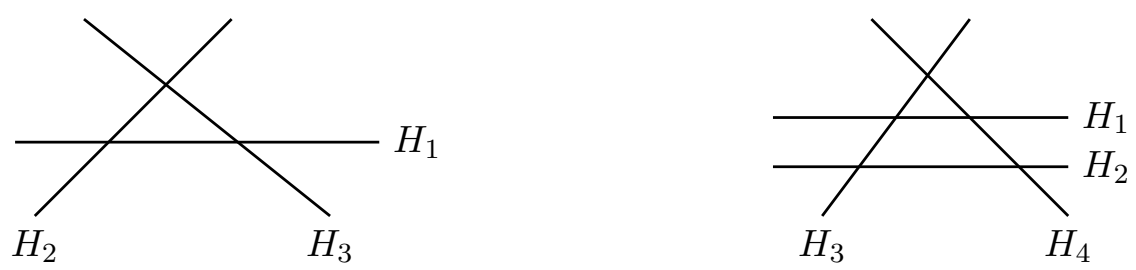

Figure 2: Elementary Arrangements in Dimension 2. 


\subsection{Distinguished Elements}

For $h=1, \ldots, m$, let $K_{h}=\left\{j_{1}, \ldots, j_{\lambda_{h}}\right\}$ be an ordered $\lambda_{h}$-element subset of $J_{h}$. Recall the notation $F_{K_{h}}=F\left(H_{j_{1}}, \ldots, H_{j_{h}}\right) \in \mathcal{F}^{\lambda_{h}}\left(\mathcal{C}_{J_{\lambda}}\right)$. The elements of the flag space $\mathcal{F}^{k}\left(\mathcal{C}_{J_{\lambda}}\right)$ of the form

$$
F_{K_{1}, \ldots, K_{m}}=F_{K_{1}} \wedge F_{K_{2}} \wedge \cdots \wedge F_{K_{m}} \in \mathcal{F}^{k}\left(\mathcal{C}_{J_{\lambda}}\right)
$$

are called the distinguished elements of the elementary arrangement $\mathcal{C}_{J_{\lambda}}$.

Lemma 3.2.1. Counted up to permutation of indices potentially changing sign, there are exactly $\prod_{h=1}^{m}\left(\lambda_{h}+\right.$ 1) distinguished elements of $\mathcal{C}_{J_{\lambda}}$.

For example, in Figure 2 the distinguished elements of the first arrangement are $\pm F_{1,2}, \pm F_{2,3}, \pm F_{1,3}$ and distinguished elements of the second are $\pm F_{1,3}, \pm F_{1,4}, \pm F_{2,3}, \pm F_{2,4}$.

Let $J_{h}=\left\{j_{1}^{h}, j_{2}^{h}, \ldots, j_{\lambda_{h}+1}^{h}\right\}$, and let $K_{\widehat{j_{i}^{h}}}=\left\{j_{1}^{h}, \ldots, \widehat{j_{i}^{h}}, \ldots, j_{\lambda_{h}+1}^{h}\right\}$ be an ordered $\lambda_{h}$-element subset of $J_{h}$. The element

$$
\begin{gathered}
s\left(\mathcal{C}_{J_{\lambda}}\right)=\left(\sum_{i=1}^{\lambda_{1}+1}(-1)^{i+1} a_{j_{i}^{1}} F_{K_{\widehat{j_{i}^{1}}}}\right) \wedge\left(\sum_{i=1}^{\lambda_{2}+1}(-1)^{i+1} a_{j_{i}^{2}} F_{K_{j_{i}^{2}}}\right) \wedge \ldots \\
\cdots \wedge\left(\sum_{i=1}^{\lambda_{m}+1}(-1)^{i+1} a_{j_{i}^{m}} F_{K_{\widehat{j_{i}^{m}}}}\right) \in \mathcal{F}^{k}\left(\mathcal{C}_{J_{\lambda}}\right)
\end{gathered}
$$

is called the singular element of $\mathcal{C}_{J_{\lambda}}$.

Lemma 3.2.2. We have $s\left(\mathcal{C}_{J_{\lambda}}\right) \in \operatorname{Sing}_{a} \mathcal{F}^{k}\left(\mathcal{C}_{J_{\lambda}}\right)$.

Proof. By Corollary 1.15.1, the element $s\left(\mathcal{C}_{J_{\lambda}}\right)$ lies in $\operatorname{Sing}_{A} \mathcal{F}^{k}\left(\mathcal{C}_{J_{\lambda}}\right)$ if and only if it is orthogonal to each of the elements $\sum_{j \in J} F_{j, i_{1}, \ldots, i_{k-1}}$ labeled by independent subsets $I=\left\{i_{1}, \ldots, i_{k-1}\right\} \subset J_{\lambda}$. If $\left\{i_{1}, \ldots, i_{k-1}\right\} \subset J_{\lambda}$ is independent, then there is $l \in\{1, \ldots, m\}$ such that $\left|I \cap J_{l}\right|=\lambda_{l}-1$ and $\left|I \cap J_{h}\right|=\lambda_{h}$ for $h \neq l$. Then it is clear that $S^{(a)}\left(s\left(\mathcal{C}_{J_{\lambda}}\right), \sum_{j \in J} F_{j, i_{1}, \ldots, i_{k-1}}\right)=0$ as the sum of two opposite terms coming from the $l$-th factor in formula (3.2).

The singular element $s\left(\mathcal{C}_{J_{\lambda}}\right)$ has the following properties. It is defined uniquely up to multiplication by \pm 1 , and this sign depends on the ordering put on each subset $J_{h}$. Each destinguished element of $\mathcal{C}_{J_{\lambda}}$, considered up to sign, enters the singular element exactly once. 
Example 2. In Figure 2 the singular element of the first elementary arrangement is $\pm\left(a_{3} F_{1,2}+a_{2} F_{3,1}+\right.$ $\left.a_{1} F_{2,3}\right)$ and the singular element of the second is $\pm\left(a_{2} F_{1}-a_{1} F_{2}\right) \wedge\left(a_{3} F_{4}-a_{4} F_{3}\right)$.

\subsection{Decomposing Determinants}

Recall the elements $g_{j}=\sum_{i=1}^{k} b_{j}^{i} t_{i} \in X^{*}$, used in defining the hyperplanes $H_{j}$, and the notation $d_{j_{1}, \ldots, j_{k}}=\operatorname{det}\left(b_{j_{\ell}}^{i}\right)_{i, \ell=1}^{k}$.

Let $s_{1}, \ldots, s_{k}$ be a basis of $X^{*}$ adjacent to the filtration $\left\{X_{h}^{*}\left(J_{\lambda}\right)\right\}_{h=1}^{m}$ in (3.1), i.e., let $s_{1}, \ldots, s_{k}$ be such that for any $h=1, \ldots, m$, the elements $s_{1}, \ldots, s_{\lambda^{h}}$ form a basis of $X_{h}^{*}\left(J_{\lambda}\right)$. Additionally, we select $s_{1}, \ldots, s_{k}$ such that the change of basis matrix from $t_{1}, \ldots, t_{k}$ to $s_{1}, \ldots, s_{k}$ has determinant one.

Let $g_{j}=\sum_{i=1}^{k} c_{j}^{i} s_{i}$, be the expansion of $g_{j}$ with respect to the new basis, then for any $j_{1}, \ldots, j_{k} \in J_{\lambda}$ we have $\operatorname{det}\left(c_{j_{\ell}}^{i}\right)_{i, \ell=1}^{k}=d_{j_{1}, \ldots, j_{k}}$ and $c_{j}^{i}=0$ for all $j \in J_{h}, i>\lambda^{h}$.

For $h=1, \ldots, m$, let $K_{h}=\left\{k_{1}^{h}, \ldots, k_{\lambda_{h}}^{h}\right\}$ be an ordered $\lambda_{h}$-element subset of $J_{h}$. Consider $K=$ $\left(K_{1}, K_{2}, \ldots, K_{m}\right)$ as an ordered $k$-element subset of $J_{\lambda}$. The matrix $\left(c_{j}^{i}\right)_{j \in K}^{i=1, \ldots, k}$ is lower block-triangular. Define the diagonal $\lambda_{h} \times \lambda_{h}$-blocks as the matrices $C_{h}=\left(c_{k_{j}^{h}}^{i}\right)_{j=1, \ldots, \lambda_{h}}^{i=\lambda^{h-1}+1, \ldots, \lambda^{h}}$.

Lemma 3.3.1. Let $K$ be constructed as above. Then, $d_{K}=\prod_{h=1}^{m} \operatorname{det} C_{h}$.

\subsection{Auxiliary Arrangements}

For $h=1, \ldots, m$, let $Y_{h}$ be a vector space of dimension $\lambda_{h}$ with coordinates $s_{1, h}, \ldots, s_{\lambda_{h}, h}$. For $j \in J_{h}$, let $g_{j, h}$ be elements of $Y_{h}^{*}$ given by the formula $g_{j, h}=\sum_{i=1}^{\lambda_{h}} c_{j, h}^{i} s_{i, h}$ where $c_{j, h}^{i}:=c_{j}^{\lambda^{h-1}+i}$ and the $c_{j}^{\lambda^{h-1}+i}$ are the coefficients introduced in Section 3.3.

Define $\mathcal{C}_{J_{\lambda}, h}=\left\{H_{j, h}\right\}_{j \in J_{h}}$ to be the following weighted arrangement of affine hyperplanes in $Y_{h}$. Each hyperplane $H_{j, h}$ has weight $a_{j}$ and is defined by the equation $g_{j, h}\left(s_{1, h}, \ldots, s_{\lambda_{h}, h}\right)+z_{j}=0$, where $z_{j}$ and $a_{j}$ are the same as in Section 3.1. We call $\mathcal{C}_{J_{\lambda}, h}$ the auxiliary arrangement of type $h$ associated with the elementary arrangement $\mathcal{C}_{J_{\lambda}}$.

For an ordered $\lambda_{h}$-element subset $I=\left\{j_{1}, \ldots, j_{\lambda_{h}}\right\} \subset J_{h}$ denote $d_{I ; h}=\operatorname{det}\left(c_{j_{l}, h}^{i}\right)_{i, l=1}^{\lambda_{h}}$. Let $J_{h}=$ $\left\{j_{1}^{h}, \ldots, j_{\lambda_{h}+1}^{h}\right\}$. By the construction, the linear combination

$\sum_{i=1}^{\lambda_{h}+1}(-1)^{i+1} d_{j_{1}, \ldots, \hat{j}_{i}, \ldots, j_{\lambda_{h}+1} ; h} g_{j_{i}^{h}}$ lies in $X_{h-1}^{*}$. Choose some numbers $e_{j}, j \in \cup_{i=1}^{h-1} J_{i}$, such that the linear 
combination

$$
\sum_{i=1}^{\lambda_{h}+1}(-1)^{i+1} d_{j_{1}, \ldots, \hat{j}_{i}, \ldots, j_{\lambda_{h}+1} ; h} g_{j_{i}^{h}}+\sum_{j \in \cup_{i=1}^{h-1} J_{i}} e_{j} g_{j}=0
$$

as an element of the space $X^{*}$. Such numbers exist since $e_{j}, j \in \cup_{i=1}^{h-1} J_{i}$, span $X_{h-1}^{*}$. Denote

$$
f_{\mathcal{C}_{J_{\lambda}, h}}=\sum_{i=1}^{\lambda_{h}+1}(-1)^{i+1} d_{j_{1}, \ldots, \hat{j}_{i}, \ldots, j_{\lambda_{h}+1} ; h^{\prime}} z_{j_{i}^{h}}+\sum_{j \in \cup_{i=1}^{h-1} J_{i}} e_{j} z_{j}
$$

We call the function

$$
P_{\mathcal{C}_{J_{\lambda}, h}}=\frac{\prod_{j \in J_{h}} a_{j}}{\left(2 \lambda_{h}\right) !} \frac{\left(f_{\mathcal{C}_{J_{\lambda}, h}}\right)^{2 \lambda_{h}}}{\left(\prod_{i=1}^{\lambda_{h}+1} d_{j_{1}^{h}, \ldots, j_{i}^{h}, \ldots, j_{\lambda_{h}+1}^{h} ; h}\right)^{2}}
$$

a prepotential of first kind of the auxiliary arrangement $\mathcal{C}_{J_{h}}$. We call the function $P_{\mathcal{C}_{J_{\lambda}}}=\prod_{h=1}^{m} P_{\mathcal{C}_{J_{\lambda}, h}}$ a prepotential of first kind of the elementary arrangement $\mathcal{C}_{J_{\lambda}}$. We call the function

$$
Q_{\mathcal{C}_{J_{\lambda}}}=\ln \left(f_{\mathcal{C}_{J_{\lambda}, 1}}\right) \prod_{h=1}^{m} P_{\mathcal{C}_{J_{\lambda}, h}}
$$

a prepotential of second kind of the elementary arrangement $\mathcal{C}_{J_{\lambda}}$. The prepotentials are not unique due to the choice of the numbers $e_{j}$ above.

\subsection{Elementary subarrangements}

Let us return to the situation of Section 2. For $x \in \mathbb{C}^{n}-\Delta$ consider the weighted arrangement $\mathcal{C}(x)$ with normal crossings.

Let $\mathcal{C}_{J_{\lambda}}(x)=\left\{H_{i}(x)\right\}_{i \in J_{\lambda}}$ be an elementary subarrangement of the arrangement $\mathcal{C}(x)$ of type $\lambda=$ $\left(\lambda_{1}, \ldots, \lambda_{m}\right)$. Recall that $J_{\lambda}=\cup_{h=1}^{m} J_{h} \subset J$ with subsets $J_{h}$ satisfying properties described in Section 3.1. According to those properties if a subarrangement $\mathcal{C}_{J_{\lambda}}(x)=\left\{H_{i}(x)\right\}_{i \in J_{\lambda}}$ is an elementary subarrangement of $\mathcal{C}(x)$ for some $x \in \mathbb{C}^{n}-\Delta$, then the subarrangement $\mathcal{C}_{J_{\lambda}}\left(x^{\prime}\right)=\left\{H_{i}\left(x^{\prime}\right)\right\}_{i \in J_{\lambda}}$, associated with the same $J_{\lambda}$, is an elementary subarrangement of $\mathcal{C}\left(x^{\prime}\right)$ for every $x^{\prime} \in \mathbb{C}^{n}-\Delta$.

Example 3. If $\mathcal{C}(x)$ is a generic arrangement, then all elementary subarrangements are of type $\lambda=(k)$, they are given by $k+1$-element subsets of $J$. 
Since $\mathcal{C}(x)$ is with normal crossings we have a natural embeddings of graded exterior algebras $\mathcal{F}\left(\mathcal{C}_{J_{\lambda}}(x)\right) \subset$ $\mathcal{F}(\mathcal{C}(x))$ and an embedding of spaces $\operatorname{Sing}_{A} \mathcal{F}^{k}\left(\mathcal{C}_{J_{\lambda}}(x)\right) \subset \operatorname{Sing}_{A} \mathcal{F}^{k}(\mathcal{C}(x))$. In particular, the singular element $s\left(\mathcal{C}_{J_{\lambda}}(x)\right)$ of $\mathcal{C}_{J_{\lambda}}(x)$ can be considered as an element of $\operatorname{Sing}_{A} \mathcal{F}^{k}(\mathcal{C}(x))$.

Recall that for $h=1, \ldots, m$, there are auxiliary arrangements $\mathcal{C}_{J_{\lambda}, h}(x)$ associated with $\mathcal{C}_{J_{\lambda}}(x)$. For $h=1, \ldots, m-1$, we define the weight of the auxiliary arrangement $\mathcal{C}_{J_{\lambda}, h}(x)$ with respect to $\mathcal{C}(x)$ as

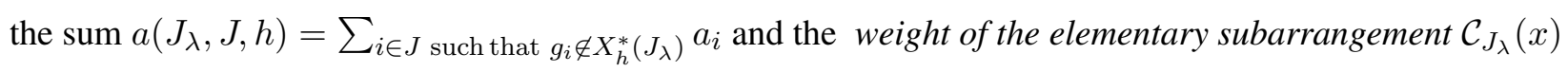
with respect to $\mathcal{C}(x)$ as the product $a\left(J_{\lambda}, J\right)=a_{J} \cdot \prod_{h=1}^{m-1} a\left(J_{\lambda}, J, h\right)$. We define the potentials of first and second kind of the family of arrangements $\mathcal{C}(x), x \in \mathbb{C}^{n}-\Delta$, to be respectively the following functions on $\mathbb{C}^{n}-\Delta$ :

$$
\begin{aligned}
P\left(x_{1}, \ldots, x_{n}\right) & =\sum \frac{1}{a\left(J_{\lambda}, J\right)} P_{\mathcal{C}_{J_{\lambda}}}\left(x_{1}, \ldots, x_{n}\right), \\
Q\left(x_{1}, \ldots, x_{n}\right) & =\sum \frac{a_{J}}{a\left(J_{\lambda}, J\right)} Q_{\mathcal{C}_{J_{\lambda}}}\left(x_{1}, \ldots, x_{n}\right),
\end{aligned}
$$

where the sums are over all elementary subarrangements $\mathcal{C}_{J_{\lambda}}(x)$ of $\mathcal{C}(x)$ and $P_{J_{\lambda}}(x), Q_{J_{\lambda}}(x)$ are the prepotentials of first and second kind, respectively, of the elementary subarrangements $\mathcal{C}_{J_{\lambda}}(x)$ of the arrangement $\mathcal{C}(x)$. The potentials are not uniques, since the prepotentials are not unique, see Section 3.4.

Example 4. The second arrangement in Figure 2 has three elementary subarrangements with $J_{\lambda}$ being $\{1,3,4\}$ or $\{2,3,4\}$ or $\{1,2\} \cup\{3,4\}$ of types $\lambda=(2),(2),(1,1)$, respectively. The potential of second kind for that arrangement is

$$
\begin{aligned}
& Q\left(z_{1}, z_{2}, z_{3}, z_{4}\right)=a_{1} a_{3} a_{4} \ln \left(f_{\{1,3,4\}, 1}\left(z_{1}, z_{2}, z_{3}\right)\right) \frac{\left(f_{\{1,3,4\}, 1}\left(z_{1}, z_{3}, z_{4}\right)\right)^{4}}{4 !\left(d_{1,3} d_{3,4} d_{4,1}\right)^{2}} \\
& +a_{2} a_{3} a_{4} \ln \left(f_{\{2,3,4\}, 1}\left(z_{2}, z_{3}, z_{4}\right)\right) \frac{\left(f_{\{2,3,4\}, 1}\left(z_{2}, z_{3}, z_{4}\right)\right)^{4}}{4 !\left(d_{2,3} d_{3,4} d_{4,2}\right)^{2}} \\
& \quad+\frac{a_{1} a_{2} a_{3} a_{4}}{a_{3}+a_{4}} \ln \left(f_{\{1,2\} \cup\{3,4\}, 1}\left(z_{1}, z_{2}\right)\right) \frac{\left(f_{\{1,2\} \cup\{3,4\}, 1}\left(z_{1}, z_{2}\right)\right)^{2}}{2 !\left(d_{1 ; 1} d_{2 ; 1}\right)^{2}} \frac{\left(f_{\{1,2\} \cup\{3,4\}, 2}\left(z_{1}, z_{2}, z_{3}, z_{4}\right)\right)^{2}}{2 !\left(d_{3 ; 2} d_{4 ; 2}\right)^{2}}
\end{aligned}
$$

c.f. this formula with the formula in the example of Section 0.2.

The potentials $P, Q$ for families of generic arrangements were constructed in [V5], c.f. [V7].

Lemma 3.5.1. For any independent subset $\left\{s_{1}, \ldots, s_{k-1}\right\} \subset J$, we have

$$
\sum_{j \in J} d_{j, s_{1}, \ldots, s_{k-1}} \frac{\partial P}{\partial z_{j}}=\sum_{j \in J} d_{j, s_{1}, \ldots, s_{k-1}} \frac{\partial Q}{\partial z_{j}}=0
$$


Proof. It is enough to prove that $\sum_{j \in J} d_{j, s_{1}, \ldots, s_{k-1}} \frac{\partial P_{\mathcal{C}_{J_{\lambda}}(z)}}{\partial z_{j}}=\sum_{j \in J} d_{j, s_{1}, \ldots, s_{k-1}} \frac{\partial Q_{\mathcal{C}_{J_{\lambda}}(z)}}{\partial z_{j}}=0$ for every elementary subarrangement $\mathcal{C}_{J_{\lambda}}(z)$ of $\mathcal{C}(x)$. To prove that, it is enough to prove that $\sum_{j \in J} d_{j, s_{1}, \ldots, s_{k-1}} \frac{\partial f_{\mathcal{C}_{J_{\lambda}, h}}}{\partial z_{j}}=$ 0 for any $h$, see formula (3.4), but that is clear. 


\section{CHAPTER 4}

\section{Orthogonal projection}

\subsection{Formula for orthogonal projection}

Recall the objects of Section 2. For $x \in \mathbb{C}^{n}-\Delta$, we denoted $V=\mathcal{F}^{k}(\mathcal{C}(x)), \operatorname{Sing}_{a} V=\operatorname{Sing}_{a} \mathcal{F}^{k}(\mathcal{C}(x))$, $F_{j_{1}, \ldots, j_{k}}=F_{j_{1}, \ldots, j_{k}}(x)$. Let $\pi: V \rightarrow \operatorname{Sing}_{a} V$ be the orthogonal projection with respect to $S^{(a)}$.

For an ordered independent subset $I=\left\{i_{1}, \ldots, i_{k}\right\} \subset J$, let $E_{I}$ be the set of all elementary subarrangements $\mathcal{C}_{J_{\lambda}}(x)$ of $\mathcal{C}(x)$ which have $F_{I}$ as a distinguished element. Let $\mathcal{C}_{J_{\lambda}}(x) \in E_{I}$ be such a subarrangement. Let $s\left(\mathcal{C}_{J_{\lambda}}(x)\right)$ be the singular element of $\mathcal{C}_{J_{\lambda}}(x)$ considered as an element of Sing ${ }_{a} V$. The singular element is defined up to multiplication by \pm 1 . We fix the sign so that the distinguished element $F_{I}$ enters $s\left(\mathcal{C}_{J_{\lambda}}(x)\right)$ with coefficient 1 .

Theorem 4.1.1. For an independent ordered subset $I=\left\{i_{1}, \ldots, i_{k}\right\} \subset J$ we have

$$
\pi\left(F_{I}\right)=\sum_{C_{J_{\lambda}}(x) \in E_{I}} \frac{1}{a\left(J_{\lambda}, J\right)} s\left(\mathcal{C}_{J_{\lambda}}(x)\right) \in \operatorname{Sing}_{a} V
$$

Corollary 4.1.2. The space Sing ${ }_{a} V$ is generated by singular elements of elementary subarrangements.

Notice that the singular element of an elementary subarrangement in $\mathbb{C}^{k}$ is a linear combination of at most $2^{k}$ basis vectors $F_{l_{1}, \ldots, l_{k}} \in V$, while the dimension of $V$ could be arbitrarily big and grow with $n$.

Example 5. For the second arrangement in Figure 2, we have

$$
\begin{gathered}
\pi\left(F_{3,4}\right)=\frac{1}{a_{1}+a_{2}+a_{3}+a_{4}}\left(\left(a_{1} F_{3,4}+a_{3} F_{4,1}+a_{4} F_{1,3}\right)+\left(a_{2} F_{3,4}+a_{3} F_{4,2}+a_{4} F_{2,3}\right)\right), \\
\pi\left(F_{2,3}\right)=\frac{1}{a_{1}+a_{2}+a_{3}+a_{4}}\left(\left(a_{4} F_{2,3}+a_{2} F_{3,4}+a_{3} F_{4,2}\right)\right. \\
\left.\quad+\frac{1}{a_{3}+a_{4}}\left(a_{1} F_{2}-a_{2} F_{1}\right) \wedge\left(a_{4} F_{3}-a_{3} F_{4}\right)\right),
\end{gathered}
$$

where $\left(a_{1} F_{2}-a_{2} F_{1}\right) \wedge\left(a_{4} F_{3}-a_{3} F_{4}\right)=a_{1} a_{4} F_{2,3}-a_{1} a_{3} F_{2,4}-a_{2} a_{4} F_{1,3}+a_{1} a_{3} F_{1,4}$. 


\subsection{Proof of Theorem 4.1.1}

Recall that every element of the form $\sum_{j \in J} F_{j, l_{1}, \ldots, l_{k-1}}$ is orthogonal to Sing ${ }_{a} V$. In order to construct $\pi\left(F_{I}\right)$ from $F_{I}$ we add to $F_{I}$ a linear combination of elements of the form $\sum_{j \in J} F_{j, l_{1}, \ldots, l_{k-1}}$ so that the result is a linear combination of the singular elements of elementary subarrangements $\mathcal{C}_{J_{\lambda}}(x)$. That means that the result lies in $\operatorname{Sing}_{a} V$ by Lemma 3.2.2.

The transition from $F_{I}$ to $\pi\left(F_{I}\right)$ is done in $k$ steps and this reasoning is by induction on the number $m$ appearing in the presentation $\lambda=\left(\lambda_{1}, \ldots, \lambda_{m}\right)$. As the first step we add to $F_{I}$ a linear combination of elements of the form $\sum_{j \in J} F_{j, l_{1}, \ldots, l_{k-1}}$ and transform $F_{I}$ to the sum

$\sum_{C_{J_{\lambda}}(x) \in E_{I}}$ with $m=1 \frac{1}{a\left(J_{\lambda}, J\right)} s\left(\mathcal{C}_{J_{\lambda}}(x)\right)+R_{1}$, where $R_{1}$ is a remainder. Then we add a new linear combination of elements of the form $\sum_{j \in J} F_{j, l_{1}, \ldots, l_{k-1}}$ and transform the result to the sum

$\sum_{C_{J_{\lambda}}(x) \in E_{I}}$ with $m \leqslant 2 \frac{1}{a\left(J_{\lambda}, J\right)} s\left(\mathcal{C}_{J_{\lambda}}(x)\right)+R_{2}$, and so on. After $m$ steps the result will be the right-hand side in (4.1) and there will be no remainder.

We illustrate that reasoning by considering the case $k=3$. We construct the orthogonal projection of the element $F_{1,2,3}$, which could be an arbitrary basis vector of $V$ after reordering hyperplanes. Formula (4.1) says

$$
\begin{aligned}
\pi\left(F_{1,2,3}\right) & =\Sigma_{1,2,3}+\Sigma_{1,2 ; 3}+\Sigma_{1,3 ; 2}+\Sigma_{2,3 ; 1}+\Sigma_{1 ; 2,3}+\Sigma_{2 ; 1,3}+\Sigma_{3 ; 1,2}+ \\
& +\Sigma_{1 ; 2 ; 3}+\Sigma_{1 ; 3 ; 2}+\Sigma_{2 ; 1 ; 3}+\Sigma_{2 ; 3 ; 1}+\Sigma_{3 ; 1 ; 2}+\Sigma_{3 ; 2 ; 1}
\end{aligned}
$$

where

$$
\begin{gathered}
\Sigma_{1,2,3}:=\frac{1}{a_{J}} \sum^{1,2,3}\left(a_{j} F_{1,2,3}-a_{1} F_{j, 2,3}+a_{2} F_{1, j, 3}-a_{3} F_{1,2, j}\right), \\
\Sigma_{1,2 ; 3}:=\frac{1}{a_{J} a(1,2)} \sum^{1,2}\left(a_{j} F_{1,2}-a_{1} F_{j, 2}+a_{2} F_{j, 1}\right) \wedge \sum^{1,2 ; 3}\left(a_{h} F_{3}-a_{3} F_{h}\right), \\
\Sigma_{1,3 ; 2}:=\frac{-1}{a_{J} a(1,3)} \sum^{1,3}\left(a_{j} F_{1,3}-a_{1} F_{j, 3}+a_{2} F_{j, 1}\right) \wedge \sum^{1,3 ; 2}\left(a_{h} F_{2}-a_{2} F_{h}\right),
\end{gathered}
$$




$$
\begin{aligned}
& \Sigma_{2,3 ; 1}:=\frac{1}{a_{J} a(2,3)} \sum^{2,3}\left(a_{j} F_{2,3}-a_{2} F_{j, 3}+a_{3} F_{j, 2}\right) \wedge \sum^{2,3 ; 1}\left(a_{h} F_{1}-a_{1} F_{h}\right), \\
& \Sigma_{1 ; 2,3}:=\frac{1}{a_{J} a(1)} \sum^{1}\left(a_{j} F_{1}-a_{1} F_{j}\right) \wedge \sum^{1 ; 2,3}\left(a_{h} F_{2,3}-a_{2} F_{h, 3}+a_{3} F_{h, 2}\right), \\
& \Sigma_{2 ; 1,3}:=\frac{-1}{a_{J} a(2)} \sum^{2}\left(a_{j} F_{2}-a_{2} F_{j}\right) \wedge \sum^{2 ; 1,3}\left(a_{h} F_{1,3}-a_{1} F_{h, 3}+a_{3} F_{h, 1}\right), \\
& \Sigma_{3 ; 1,2}:=\frac{1}{a_{J} a(3)} \sum^{3}\left(a_{j} F_{3}-a_{3} F_{j}\right) \wedge \sum^{3 ; 1,2}\left(a_{h} F_{1,2}-a_{1} F_{h, 2}+a_{2} F_{h, 1}\right), \\
& \Sigma_{1 ; 2 ; 3}:=\frac{1}{a_{J} a(1) a(1,2)} \sum^{1}\left(a_{j} F_{1}-a_{1} F_{j}\right) \wedge \sum^{1 ; 2}\left(a_{h} F_{2}-a_{2} F_{h}\right) \wedge \sum^{1,2 ; 3}\left(a_{i} F_{3}-a_{3} F_{i}\right), \\
& \Sigma_{1 ; 3 ; 2}:=\frac{-1}{a_{J} a(1) a(1,3)} \sum^{1}\left(a_{j} F_{1}-a_{1} F_{j}\right) \wedge \sum^{1 ; 3}\left(a_{h} F_{3}-a_{3} F_{h}\right) \wedge \sum^{1,3 ; 2}\left(a_{i} F_{2}-a_{2} F_{i}\right), \\
& \Sigma_{2 ; 3 ; 1}:=\frac{1}{a_{J} a(2) a(2,3)} \sum^{2}\left(a_{j} F_{2}-a_{2} F_{j}\right) \wedge \sum^{2 ; 3}\left(a_{h} F_{3}-a_{3} F_{h}\right) \wedge \sum^{2,3 ; 1}\left(a_{i} F_{1}-a_{1} F_{i}\right), \\
& \Sigma_{2 ; 1 ; 3}:=\frac{-1}{a_{J} a(2) a(2,1)} \sum^{2}\left(a_{j} F_{2}-a_{2} F_{j}\right) \wedge \sum^{2 ; 1}\left(a_{h} F_{1}-a_{1} F_{h}\right) \wedge \sum^{2,1 ; 3}\left(a_{i} F_{3}-a_{3} F_{i}\right), \\
& \Sigma_{3 ; 2 ; 1}:=\frac{-1}{a_{J} a(3) a(2,3)} \sum^{3}\left(a_{j} F_{3}-a_{3} F_{j}\right) \wedge \sum^{3 ; 2}\left(a_{h} F_{2}-a_{2} F_{h}\right) \wedge \sum^{3,2 ; 1}\left(a_{i} F_{1}-a_{1} F_{i}\right), \\
& \Sigma_{3 ; 1 ; 2}:=\frac{1}{a_{J} a(3) a(1,3)} \sum^{3}\left(a_{j} F_{3}-a_{3} F_{j}\right) \wedge \sum^{3 ; 1}\left(a_{h} F_{1}-a_{1} F_{h}\right) \wedge \sum^{3,1 ; 2}\left(a_{i} F_{2}-a_{2} F_{i}\right) .
\end{aligned}
$$

In these formulas we use the following notations.

We denote $a(h, l)=\sum a_{j}$, where the sum is over all $j \in J$ such that $g_{j} \notin \operatorname{span}\left(g_{h}, g_{l}\right)$. We denote $a(h)=\sum a_{j}$, where the sum is over all $j \in J$ such that $g_{j} \notin \operatorname{span}\left(g_{h}\right)$. 
The sum $\sum^{1,2,3}$ is over all $j \in J$ such that the subset $\{j, 1,2,3\}$ forms a circuit in $J$. The sum $\sum^{h, l}$ is over all $j \in J$ such that the subset $\{j, h, l\}$ forms a circuit in $J$. The sum $\sum^{h}$ is over all $j \in J$ such that the subset $\{j, h\}$ forms a circuit in $J$.

The $\operatorname{sum} \sum^{j, l ; s}$ is over all $h \in J$ such that $g_{h} \notin \operatorname{span}\left(g_{j}, g_{l}\right)$. The sum $\sum^{j ; l, s}$ is over all $h \in J$ such that $\operatorname{span}\left(g_{j}, g_{l}, g_{h}\right)=\operatorname{span}\left(g_{j}, g_{s}, g_{h}\right)=\left(\mathbb{C}^{3}\right)^{*}$. The sum $\sum^{j ; l}$ is over all $h \in J$ such that $\operatorname{span}\left(g_{j}, g_{l}\right)=$ $\operatorname{span}\left(g_{j}, g_{h}\right)$.

The first transformation is

$$
F_{1,2,3} \mapsto F_{1,2,3}-\frac{a_{1}}{a_{J}} \sum_{j \in I} F_{j, 2,3}-\frac{a_{2}}{a_{J}} \sum_{j \in I} F_{1, j, 3}-\frac{a_{3}}{a_{J}} \sum_{j \in I} F_{1,2, j}
$$

the added terms are a linear combination of elements of the form $\sum_{j \in J} F_{j, l_{1}, l_{2}}$. We rearrange the right-hand side of (4.3) as follows:

$$
\begin{aligned}
& =a_{J}^{-1} \sum^{1,2,3}\left(a_{j} F_{1,2,3}-a_{1} F_{j, 2,3}+a_{2} F_{1, j, 3}-a_{3} F_{1,2, j}\right) \\
& +a_{J}^{-1} \sum^{1,2}\left(a_{j} F_{1,2}-a_{1} F_{j, 2}+a_{2} F_{j, 1}\right) \wedge F_{3} \\
& -a_{J}^{-1} \sum^{1,3}\left(a_{j} F_{1,3}-a_{1} F_{j, 3}+a_{3} F_{j, 1}\right) \wedge F_{2} \\
& +a_{J}^{-1} \sum^{2,3}\left(a_{j} F_{2,3}-a_{2} F_{j, 3}+a_{3} F_{j, 2}\right) \wedge F_{1} \\
& +a_{J}^{-1} \sum^{1}\left(a_{j} F_{1}-a_{1} F_{j}\right) \wedge F_{2,3}-a_{J}^{-1} \sum^{2}\left(a_{j} F_{2}-a_{2} F_{j}\right) \wedge F_{1,3} \\
& +a_{J}^{-1} \sum^{3}\left(a_{j} F_{3}-a_{3} F_{j}\right) \wedge F_{1,2} .
\end{aligned}
$$

The sum in (4.4) is exactly the sum $\sum_{C_{J_{\lambda}}(x) \in E_{\{1,2,3\}} \text { with } m=1} \frac{1}{a\left(J_{\lambda}, J\right)} s\left(\mathcal{C}_{J_{\lambda}}(x)\right)$ and the sums in (4.5)-(4.9) form the first remainder $R_{1}$.

Now we add to each of the sums in (4.5)-(4.9) a linear combination of elements of the form $\sum_{j \in J} F_{j, l_{1}, l_{2}}$ as follows. Let

$$
\begin{aligned}
& a_{J}^{-1} \sum^{1,2}\left(a_{j} F_{1,2}-a_{1} F_{j, 2}+a_{2} F_{j, 1}\right) \wedge F_{3} \rightarrow \\
& a_{J}^{-1} \sum^{1,2}\left(a_{j} F_{1,2}-a_{1} F_{j, 2}+a_{2} F_{j, 1}\right) \wedge\left(F_{3}-\frac{a_{3}}{a(1,2)} \sum_{i \in J} F_{i}\right) \\
& =\frac{1}{a_{J} a(1,2)} \sum^{1,2}\left(a_{j} F_{1,2}-a_{1} F_{j, 2}+a_{2} F_{j, 1}\right) \wedge \sum^{1,2 ; 3}\left(a_{i} F_{3}-a_{3} F_{i}\right) .
\end{aligned}
$$


Similarly,

$$
\begin{aligned}
& -a_{J}^{-1} \sum^{1,3}\left(a_{j} F_{1,3}-a_{1} F_{j, 3}+a_{3} F_{j, 1}\right) \wedge F_{2} \rightarrow \\
& -\frac{1}{a_{J} a(1,3)} \sum^{1,3}\left(a_{j} F_{1,3}-a_{1} F_{j, 3}+a_{2} F_{j, 1}\right) \wedge \sum^{1,3 ; 2}\left(a_{i} F_{2}-a_{2} F_{i}\right), \\
& a_{J}^{-1} \sum^{2,3}\left(a_{j} F_{2,3}-a_{2} F_{j, 3}+a_{3} F_{j, 2}\right) \wedge F_{1} \rightarrow \\
& \frac{1}{a_{J} a(2,3)} \sum^{2,3}\left(a_{j} F_{2,3}-a_{2} F_{j, 3}+a_{3} F_{j, 2}\right) \wedge \sum^{2,3 ; 1}\left(a_{i} F_{1}-a_{1} F_{i}\right) .
\end{aligned}
$$

Similarly

$$
\begin{aligned}
& a_{J}^{-1} \sum^{1}\left(a_{j} F_{1}-a_{1} F_{j}\right) \wedge F_{2,3} \rightarrow \\
& a_{J}^{-1} \sum^{1}\left(a_{j} F_{1}-a_{1} F_{j}\right) \wedge\left(F_{2,3}-\frac{a_{2}}{a(1)} \sum_{i \in J} F_{i, 3}-\frac{a_{3}}{a(1)} \sum_{i \in J} F_{2, i}\right) \\
& =\frac{1}{a_{J} a(1)} \sum^{1}\left(a_{j} F_{1}-a_{1} F_{j}\right) \wedge \sum^{1 ; 2,3}\left(a_{i} F_{2,3}-a_{2} F_{i, 3}+a_{3} F_{i, 2}\right) \\
& \quad+\frac{1}{a_{J} a(1)} \sum^{1}\left(a_{j} F_{1}-a_{1} F_{j}\right) \wedge \sum^{1 ; 2}\left(a_{i} F_{2}-a_{2} F_{i}\right) \wedge F_{3} \\
& \quad-\frac{1}{a_{J} a(1)} \sum^{1}\left(a_{j} F_{1}-a_{1} F_{j}\right) \wedge \sum^{1 ; 3}\left(a_{i} F_{3}-a_{3} F_{i}\right) \wedge F_{2} .
\end{aligned}
$$

We transform similarly the remaining two sums in (4.8)-(4.9). This finishes step two of the procedure. After the two steps the result is

$$
\begin{aligned}
& \Sigma_{1,2,3}+\Sigma_{1,2 ; 3}+\Sigma_{1,3 ; 2}+\Sigma_{2,3 ; 1}+\Sigma_{1 ; 2,3}+\Sigma_{2 ; 1,3}+\Sigma_{3 ; 1,2} \\
& +\frac{1}{a_{J} a(1)} \sum^{1}\left(a_{j} F_{1}-a_{1} F_{j}\right) \wedge \sum^{1 ; 2}\left(a_{i} F_{2}-a_{2} F_{i}\right) \wedge F_{3} \\
& -\frac{1}{a_{J} a(1)} \sum^{1}\left(a_{j} F_{1}-a_{1} F_{j}\right) \wedge \sum^{1 ; 3}\left(a_{i} F_{3}-a_{3} F_{i}\right) \wedge F_{2} \\
& -\frac{1}{a_{J} a(2)} \sum^{2}\left(a_{j} F_{2}-a_{2} F_{j}\right) \wedge \sum^{2 ; 1}\left(a_{i} F_{1}-a_{1} F_{i}\right) \wedge F_{3} \\
& +\frac{1}{a_{J} a(2)} \sum^{2}\left(a_{j} F_{2}-a_{2} F_{j}\right) \wedge \sum^{2 ; 3}\left(a_{i} F_{3}-a_{3} F_{i}\right) \wedge F_{1}
\end{aligned}
$$




$$
\begin{aligned}
& -\frac{1}{a_{J} a(3)} \sum^{3}\left(a_{j} F_{3}-a_{3} F_{j}\right) \wedge \sum^{3 ; 2}\left(a_{i} F_{2}-a_{2} F_{i}\right) \wedge F_{1} \\
& +\frac{1}{a_{J} a(3)} \sum^{3}\left(a_{j} F_{3}-a_{3} F_{j}\right) \wedge \sum^{3 ; 1}\left(a_{i} F_{1}-a_{1} F_{i}\right) \wedge F_{2} .
\end{aligned}
$$

The sum in (4.10) is the sum $\sum_{C_{J_{\lambda}}(x) \in E_{\{1,2,3\}} \text { with } m \leqslant 2} \frac{1}{a\left(J_{\lambda}, J\right)} s\left(\mathcal{C}_{J_{\lambda}}(x)\right)$ and the sums in (4.11)-(4.16) form the second remainder $R_{2}$. As the third step we transform the expression in (4.11) to

$$
\begin{aligned}
& \frac{1}{a_{J} a(1)} \sum^{1}\left(a_{j} F_{1}-a_{1} F_{j}\right) \wedge \sum^{1 ; 2}\left(a_{i} F_{2}-a_{2} F_{i}\right) \wedge\left(F_{3}-\frac{a_{3}}{a(1,2)} \sum_{l \in J} F_{l}\right) \\
& =\frac{1}{a_{J} a(1) a(1,2)} \sum^{1}\left(a_{j} F_{1}-a_{1} F_{j}\right) \wedge \sum^{1 ; 2}\left(a_{i} F_{2}-a_{2} F_{i}\right) \wedge \sum^{1,2 ; 3}\left(a_{l} F_{3}-a_{3} F_{l}\right),
\end{aligned}
$$

and similarly we transform the expressions in (4.12)-(4.16). After these three steps we obtain formula (4.2). The case of arbitrary $k$ is similar to this case of $k=3$. Theorem 4.1 .1 is proved.

\subsection{Potential of first kind}

Recall the objects of Section 2. Recall that $v_{j_{1}, \ldots, j_{k}}=\pi\left(F_{j_{1}, \ldots, j_{k}}\right)$, where $\pi: V \rightarrow \operatorname{Sing}_{a} V$ is the orthogonal projection with respect to $S^{(a)}$. Let $P$ be the potential of first kind of the family of arrangements $\mathcal{C}(x), x \in \mathbb{C}^{n}-\Delta$.

Theorem 4.3.1. For any two ordered independent subsets $I=\left\{i_{1}, \ldots, i_{k}\right\}, L=\left\{l_{1}, \ldots, l_{k}\right\} \subset J$, we have

$$
S^{(a)}\left(v_{i_{1}, \ldots, i_{k}}, v_{l_{1}, \ldots, l_{k}}\right)=d_{i_{1}, \ldots, i_{k}} d_{l_{1}, \ldots, l_{k}} \frac{\partial^{2 k} P}{\partial z_{i_{1}} \ldots \partial z_{i_{k}} \partial z_{l_{1}} \ldots \partial z_{l_{k}}} .
$$

For families of generic arrangements this theorem was proved in [V5], c.f. [V7].

Proof. Since $v_{l_{1}, \ldots, l_{k}}=\pi\left(F_{l_{1}, \ldots, l_{k}}\right)$ we have

$$
\begin{aligned}
S^{(a)}\left(v_{i_{1}, \ldots, i_{k}}, v_{l_{1}, \ldots, l_{k}}\right) & =S^{(a)}\left(v_{i_{1}, \ldots, i_{k}}, F_{l_{1}, \ldots, l_{k}}\right) \\
& =\sum_{C_{J_{\lambda}}(x) \in E_{i_{1}, \ldots, i_{k}}} \frac{1}{a\left(J_{\lambda}, J\right)} S^{(a)}\left(s\left(\mathcal{C}_{J_{\lambda}}(x)\right), F_{l_{1}, \ldots, l_{k}}\right) .
\end{aligned}
$$

Formula (3.2) for the singular element $s\left(\mathcal{C}_{J_{\lambda}}(x)\right)$ shows that the number $S^{(a)}\left(s\left(\mathcal{C}_{J_{\lambda}}(x)\right), F_{l_{1}, \ldots, l_{k}}\right)$ is nonzero if and only if $F_{l_{1}, \ldots, l_{k}}$ is a distinguished elements of the elementary arrangement $\mathcal{C}_{J_{\lambda}}(x)$. Let this condition be satisfied for an elementary arrangement $\mathcal{C}_{J_{\lambda}}(x) \in E_{i_{1}, \ldots, i_{k}}$. Let $J_{\lambda}=\cup_{h=1}^{m} J_{h}, J_{h}=\left\{j_{1}^{h}, j_{2}^{h}, \ldots, j_{\lambda_{h}+1}^{h}\right\}$, 
and let $K_{\widehat{j_{i}^{h}}}=\left\{j_{1}^{h}, \ldots, \widehat{j_{i}^{h}}, \ldots, j_{\lambda_{h}+1}^{h}\right\}$ be an ordered $\lambda_{h}$-element subset of $J_{h}$. Then

$$
\begin{gathered}
s\left(\mathcal{C}_{J_{\lambda}}(x)\right)=\left(\sum_{i=1}^{\lambda_{1}+1}(-1)^{i+1} a_{j_{i}^{1}} F_{K_{\widehat{j_{i}^{1}}}}\right) \wedge\left(\sum_{i=1}^{\lambda_{2}+1}(-1)^{i+1} a_{j_{i}^{2}} F_{K_{\widehat{j_{i}^{2}}}}\right) \wedge \ldots \\
\cdots \wedge\left(\sum_{i=1}^{\lambda_{m}+1}(-1)^{i+1} a_{j_{i}^{m}} F_{K_{\widehat{j_{i}^{m}}}}\right) .
\end{gathered}
$$

Due to the choice of sign of $s\left(\mathcal{C}_{J_{\lambda}}(x)\right)$ in Section 4.1 we may assume that

$$
F_{i_{1}, \ldots, i_{k}}=F_{K_{\widehat{j_{1}^{1}}}} \wedge F_{K_{\widehat{j_{1}^{2}}}} \wedge \ldots F_{K_{\widehat{j_{1}^{m}}}}
$$

We may also assume that

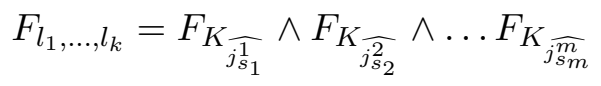

for some $s_{h} \in\left\{1, \ldots, \lambda_{h}+1\right\}, h=1, \ldots, m$. $^{(1)}$

Equations (4.18)-(4.20) imply

$$
S^{(a)}\left(s\left(\mathcal{C}_{J_{\lambda}}(x)\right), F_{l_{1}, \ldots, l_{k}}\right)=\prod_{h=1}^{m}(-1)^{s_{h}+1} \prod_{h=1}^{m} \prod_{j \in J_{h}} a_{j} .
$$

Recall formula (3.7) for the potential of first kind. It is a linear combination of prepotentials of first kind $P_{\mathcal{C}_{J_{\lambda}}}(x)$ of all elementary subarrangements $\mathcal{C}_{J_{\lambda}}(x)$ of $\mathcal{C}(x)$. To finish the proof of Theorem 4.3 .1 we need to show that if $\mathcal{C}_{J_{\lambda}}(x)$ is as in formula (4.21), then

$$
d_{i_{1}, \ldots, i_{k}} d_{l_{1}, \ldots, l_{k}} \frac{\partial^{2 k} P_{\mathcal{C}_{J_{\lambda}}}}{\partial z_{i_{1}} \ldots \partial z_{i_{k}} \partial z_{l_{1}} \ldots \partial z_{i_{k}}}=\prod_{h=1}^{m}(-1)^{s_{h}+1} \prod_{h=1}^{m} \prod_{i \in J_{h}} a_{i}
$$

and

$$
\frac{\partial^{2 k} P_{\mathcal{C}_{J_{\lambda}}}}{\partial z_{i_{1}} \ldots \partial z_{i_{k}} \partial z_{l_{1}} \ldots \partial z_{i_{k}}}=0
$$

\footnotetext{
(1) Notice that if the indices of $F_{l_{1}, \ldots, l_{k}}$ are permuted then $F_{l_{1}, \ldots, l_{k}}$ is multiplied by \pm 1 and $d_{l_{1}, \ldots, l_{k}}$ in the right-hand side of (4.17)
} is multiplied by the same \pm 1 . 
if $\mathcal{C}_{J_{\lambda}}(x) \notin E_{i_{1}, \ldots, i_{k}}$ or if $\mathcal{C}_{J_{\lambda}}(x) \in E_{i_{1}, \ldots, i_{k}}$ but $F_{l_{1}, \ldots, l_{k}}$ is not a distinguished element of $\mathcal{C}_{J_{\lambda}}(x)$.

Recall the formula

$$
P_{\mathcal{C}_{J_{\lambda}}}=\prod_{h=1}^{m} \frac{\prod_{j \in J_{h}} a_{j}}{\left(2 \lambda_{h}\right) !} \frac{\left(f_{\mathcal{C}_{J_{\lambda}, h}}\right)^{2 \lambda_{h}}}{\left(\prod_{i=1}^{\lambda_{h}+1} d_{j_{1}^{h}, \ldots, \widehat{j_{i}^{h}}, \ldots, j_{\lambda_{h}+1}^{h} ; h}\right)^{2}}
$$

Lemma 4.3.2. The $2 \lambda_{h}$-th derivative of $\frac{1}{\left(2 \lambda_{h}\right) !} \frac{\left(f_{\mathcal{C}_{J_{\lambda}, h}}\right)^{2 \lambda_{h}}}{\left(\prod_{i=1}^{\lambda_{h}+1} d_{j_{1}^{h}, \ldots, j_{i}^{h}, \ldots, i_{\lambda_{h}+1}^{h} ; h}\right)^{2}}$ with respect to the variables

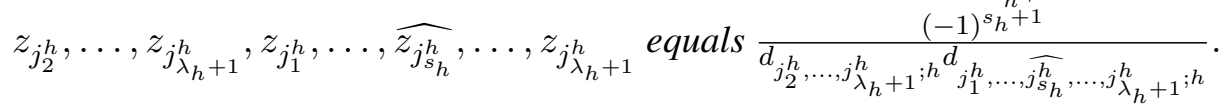

Now Lemmas 4.3.2 and 3.3.1 imply formula (4.22).

Lemma 4.3.3. Formula (4.23) holds if $\mathcal{C}_{J_{\lambda}}(x) \notin E_{i_{1}, \ldots, i_{k}}$ or if $\mathcal{C}_{J_{\lambda}}(x) \in E_{i_{1}, \ldots, i_{k}}$ but $F_{l_{1}, \ldots, l_{k}}$ is not a distinguished element of $\mathcal{C}_{J_{\lambda}}(x)$.

Proof. Let $\mathcal{C}_{J_{\lambda}}$ be any elementary subarrangement and $F_{i_{1}, \ldots, i_{k}}, F_{l_{1}, \ldots, l_{k}}$ two nonzero elements. Clearly formula (4.23) holds if $\left\{i_{1}, \ldots, i_{k}, l_{1}, \ldots, l_{k}\right\} \not \subset \cup_{h=1}^{m} J_{h}$. Assume that $\left\{i_{1}, \ldots, i_{k}, l_{1}, \ldots, l_{k}\right\} \subset \cup_{h=1}^{m} J_{h}$. For $h=1, \ldots, m$ denote $i^{h}=\left|\left\{s \mid i_{s} \in J_{h}\right\}\right|$ and $l^{h}=\left|\left\{s \mid l_{s} \in J_{h}\right\}\right|$. We have $i^{1}+\cdots+i^{h} \leqslant \lambda_{1}+\cdots+\lambda_{h}$, $l^{1}+\cdots+l^{h} \leqslant \lambda_{1}+\cdots+\lambda_{h}$ for any $h$ and $i^{1}+\cdots+i^{m}=l^{1}+\cdots+l^{m}=\lambda_{1}+\cdots+\lambda_{m}=k$. If $i^{h}=l^{h}=\lambda_{h}$ for all $h$, then $F_{i_{1}, \ldots, i_{k}}, F_{l_{1}, \ldots, l_{k}}$ are distinguished elements of $\mathcal{C}_{J_{\lambda}}$. So we assume that at least one of the numbers $i^{h}, l^{h}$ differs from $\lambda_{h}$. Let $h^{\max }$ be the maximal $h$ such that at least one of the numbers $i^{h}, l^{h}$ differs from $\lambda_{h}$. Then: (a) each of $i^{h^{\max }}, l^{h^{\max }}$ is not less than $\lambda_{h^{\max }}$; (b) at least one of them is greater than $\lambda_{h^{\max }}$; (c) $h^{\max }>1$.

Then the derivative in (4.23) is zero due to the fact that the set $\left\{i_{1}, \ldots, i_{k}, l_{1}, \ldots, l_{k}\right\}$ has too many elements of $\cup_{h=h^{\max }}^{m} J_{h}$, c.f. formulas (3.4), (3.5).

Theorem 4.3.1 is proved.

Recall the elements $p_{j} \in \mathcal{O}\left(C_{\mathcal{C}(x), a}\right), j \in J$, and the Grothiendick residue bilinear form $(,)_{C_{\mathcal{C}(x), a}}$ on $\mathcal{O}\left(C_{\mathcal{C}(x), a}\right)$.

Corollary 4.3.4. For any two independent subsets $I=\left\{i_{1}, \ldots, i_{k}\right\}, L=\left\{l_{1}, \ldots, l_{k}\right\} \subset J$, we have

$$
\left(p_{i_{1}} \ldots, p_{i_{k}}, p_{l_{1}} \ldots p_{l_{k}}\right)_{C_{\mathcal{C}(x), a}}=(-1)^{k} \frac{\partial^{2 k} P}{\partial z_{i_{1}} \ldots \partial z_{i_{k}} \partial z_{l_{1}} \ldots \partial z_{i_{k}}} .
$$


Proof. Recall that the isomorphism of vector spaces

$$
\nu(x): \operatorname{Sing}_{a} \mathcal{F}^{k}(\mathcal{C}(x)) \rightarrow \mathcal{O}\left(C_{\mathcal{C}(x), a}\right)
$$

sends $\pi\left(F_{i_{1}, \ldots, i_{k}}\right)$ to $d_{i_{1}, \ldots, i_{k}} p_{i_{1}} \ldots p_{i_{k}}$ for all independent subsets $\left\{i_{1}, \ldots, i_{k}\right\} \subset J$ and also identifies the form $S^{(a)}($,$) on \operatorname{Sing}_{a} \mathcal{F}^{k}(\mathcal{C}(x))$ and the the form $(-1)^{k}(,)_{C_{\mathcal{C}(x), a}}$ on $\mathcal{O}\left(C_{\mathcal{C}(x), a}\right)$, see Theorem 1.12.1.

\subsection{Potential of second kind}

Recall the objects of Section 2. Let $Q$ be the potential of second kind of the family of arrangements $\mathcal{C}(x), x \in \mathbb{C}^{n}-\Delta$.

Theorem 4.4.1. Let $x \in \mathbb{C}^{n}-\Delta$. Then for any two independent subsets $I=\left\{i_{1}, \ldots, i_{k}\right\}, L=\left\{l_{1}, \ldots, l_{k}\right\} \subset$ $J$ and any $i_{0} \in J$, we have

$$
\left(p_{i_{0}} p_{i_{1}} \ldots p_{i_{k}}, p_{l_{1}} \ldots p_{l_{k}}\right)_{C_{\mathcal{C}(x), a}}=(-1)^{k} \frac{\partial^{2 k+1} Q}{\partial z_{i_{0}} \partial z_{i_{1}} \ldots \partial z_{i_{k}} \partial z_{l_{1}} \ldots \partial z_{l_{k}}}(x)
$$

For families of generic arrangements this theorem was proved in [V5].

Proof. Due to relations (2.8) and (3.9) it is enough to prove (4.26) in the case when $i_{0}, i_{1}, \ldots, i_{k}$ are distinct elements of $J$. Thus assume that $i_{0}, i_{1}, \ldots, i_{k}$ are distinct. After reordering $i_{0}, i_{1}, \ldots, i_{k}$, we may assume that $i_{0}, i_{1}, \ldots, i_{\mu}$ form a circuit, where $\mu$ is some number $\leqslant k$. Recall the function

$$
f_{i_{0}, i_{1}, \ldots, i_{k}}(z)=\sum_{j=0}^{k}(-1)^{j} z_{i_{j}} d_{i_{0}, \ldots, \widehat{i_{j}, \ldots, i_{k}}}=\sum_{j=0}^{\mu}(-1)^{j} z_{i_{j}} d_{i_{0}, \ldots, \widehat{i_{j}, \ldots, i_{\mu+1}, \ldots, i_{k}}}
$$

in (2.9) and relation (2.11):

$$
p_{i_{0}} p_{i_{1}} \ldots p_{i_{k}}=\frac{1}{f_{i_{0}, i_{1}, \ldots, i_{k}}} \sum_{j=0}^{\mu}(-1)^{j} a_{i_{j}} w_{i_{0}, \ldots, \hat{i_{j}, \ldots, i_{\mu+1}, \ldots, i_{k}}} .
$$


First we analyze the left-hand side in (4.26). We have

$$
\begin{aligned}
& \left(p_{i_{0}} p_{i_{1}} \ldots p_{i_{k}}, p_{l_{1}} \ldots p_{l_{k}}\right)_{C_{\mathcal{C}(x), a}} \\
& =\frac{1}{f_{i_{0}, i_{1}, \ldots, i_{k}}}\left(\sum_{j=0}^{\mu}(-1)^{j} a_{i_{j}} w_{i_{0}, \ldots, \hat{i_{j}}, \ldots, i_{\mu+1}, \ldots, i_{k}}, p_{l_{1}} \ldots p_{l_{k}}\right)_{C_{\mathcal{C}(x), a}} \\
& =\frac{1}{d_{l_{1}, \ldots, l_{k}} f_{i_{0}, i_{1}, \ldots, i_{k}}}\left(\sum_{j=0}^{\mu}(-1)^{j} a_{i_{j}} w_{i_{0}, \ldots, \hat{i_{j}}, \ldots, i_{\mu+1}, \ldots, i_{k}}, w_{l_{1}, \ldots, l_{k}}\right)_{C_{\mathcal{C}(x), a}} \\
& =\frac{(-1)^{k}}{d_{l_{1}, \ldots, l_{k}} f_{i_{0}, i_{1}, \ldots, i_{k}}} S^{(a)}\left(\sum_{j=0}^{\mu}(-1)^{j} a_{i_{j}} v_{i_{0}, \ldots, \hat{i_{j}}, \ldots, i_{\mu+1}, \ldots, i_{k}}, v_{l_{1}, \ldots, l_{k}}\right),
\end{aligned}
$$

where the last equality holds by Theorem 1.12.1. We have

$$
\begin{aligned}
& S^{(a)}\left(\sum_{j=0}^{\mu}(-1)^{j} a_{i_{j}} v_{i_{0}, \ldots, \hat{i_{j}}, \ldots, i_{\mu+1}, \ldots, i_{k}}, v_{l_{1}, \ldots, l_{k}}\right) \\
& =S^{(a)}\left(\pi\left(\sum_{j=0}^{\mu}(-1)^{j} a_{i_{j}} F_{i_{0}, \ldots, \hat{i_{j}}, \ldots, i_{\mu+1}, \ldots, i_{k}}\right), F_{l_{1}, \ldots, l_{k}}\right),
\end{aligned}
$$

where $\pi$ is the orthogonal projection, see Section 4.1.

Let $E\left(i_{0}, i_{1}, \ldots, i_{\mu}\right)$ be the set of all elementary subarrangements $\mathcal{C}_{J_{\lambda}}(x)$ of $\mathcal{C}(x)$ with $J_{\lambda}=\cup_{h=1}^{m} J_{h}$ such that $J_{1}=\left\{i_{0}, i_{1}, \ldots, i_{\mu}\right\}$ and such that $F_{i_{1}, \ldots, i_{k}}$ is a distinguished element of $\mathcal{C}_{J_{\lambda}}(x)$. Let $s\left(\mathcal{C}_{J_{\lambda}}(x)\right)$ be the singular element of $\mathcal{C}_{J_{\lambda}}(x)$ considered as an element of $\operatorname{Sing}_{a} V$. The singular element is defined up to multiplication by \pm 1 . We fix the sign so that the distinguished element $F_{i_{1}, \ldots, i_{k}}$ enters $s\left(\mathcal{C}_{J_{\lambda}}(x)\right)$ with coefficeint 1 .

Lemma 4.4.2. We have

$$
\pi\left(\sum_{j=0}^{\mu}(-1)^{j} a_{i_{j}} F_{i_{0}, \ldots, \hat{i_{j}}, \ldots, i_{\mu+1}, \ldots, i_{k}}\right)=\sum_{\mathcal{C}_{J_{\lambda}}(x) \in E\left(i_{0}, i_{1}, \ldots, i_{\mu}\right)} \frac{a_{J}}{a\left(J_{\lambda}, J\right)} s\left(\mathcal{C}_{J_{\lambda}}(x)\right) .
$$

Proof. Indeed we have

$$
\sum_{j=0}^{\mu}(-1)^{j} a_{i_{j}} F_{i_{0}, \ldots, \widehat{i_{j}}, \ldots, i_{\mu+1}, \ldots, i_{k}}=\left(\sum_{j=0}^{\mu}(-1)^{j} a_{i_{j}} F_{i_{0}, \ldots, \hat{i_{j}}, \ldots, i_{\mu}}\right) \wedge F_{i_{\mu+1}, \ldots, i_{k}} .
$$

To construct the orthogonal projection of the right-hand side in (4.29), we need to apply the construction of the orthogonal projection, described in the proof of Theorem 4.1.1, but starting with step 2 since the result of the first step is already presented by the factor $\left(\sum_{j=0}^{\mu}(-1)^{j} a_{i_{j}} F_{i_{0}, \ldots, \widehat{i_{j}, \ldots, i_{\mu}}}\right)$ in the right-hand side of (4.29), c.f. formulas (4.4)-(4.9). 
By Lemma 4.4.2 the expression in (4.27) equals

$$
\frac{(-1)^{k}}{d_{l_{1}, \ldots, l_{k}} f_{i_{0}, i_{1}, \ldots, i_{k}}} \sum_{\mathcal{C}_{J_{\lambda}}(x) \in E\left(i_{0}, i_{1}, \ldots, i_{\mu}\right)} \frac{a_{J}}{a\left(J_{\lambda}, J\right)} S^{(a)}\left(s\left(\mathcal{C}_{J_{\lambda}}(x)\right), F_{l_{1}, \ldots, l_{k}}\right)
$$

We have

$$
\begin{aligned}
s\left(\mathcal{C}_{J_{\lambda}}(x)\right)= & \left(\sum_{j=0}^{\mu}(-1)^{j} a_{i_{j}} F_{i_{0}, \ldots, \hat{j_{j}}, \ldots, i_{\mu}}\right) \wedge\left(\sum_{i=1}^{\lambda_{2}+1}(-1)^{i+1} a_{j_{i}^{2}} F_{K_{\widehat{j_{i}^{2}}}}\right) \wedge \ldots \\
& \cdots \wedge\left(\sum_{i=1}^{\lambda_{m}+1}(-1)^{i+1} a_{j_{i}^{m}} F_{K_{\widehat{j_{i}^{m}}}}\right)
\end{aligned}
$$

where we use the notations of Section 3.2, namely, we have $J_{h}=\left\{j_{1}^{h}, j_{2}^{h}, \ldots, j_{\lambda_{h}+1}^{h}\right\}$ for $h=2, \ldots, m$, and $K_{\widehat{j_{i}^{h}}}=\left\{j_{1}^{h}, \ldots, \widehat{j_{i}^{h}}, \ldots, j_{\lambda_{h}+1}^{h}\right\}$.

Due to our choice of sign of $s\left(\mathcal{C}_{J_{\lambda}}(x)\right)$ we may assume that we have the equality of ordered sets

$$
\left\{i_{1}, \ldots, i_{k}\right\}=\left\{i_{1}, \ldots, i_{\mu}, K_{\widehat{j_{1}^{2}}}, K_{\widehat{j_{1}^{3}}}, \ldots, K_{\widehat{j_{1}^{m}}}\right\}
$$

The term $S^{(a)}\left(s\left(\mathcal{C}_{J_{\lambda}}(x)\right), F_{l_{1}, \ldots, l_{k}}\right)$ is nonzero if and only if

$$
\left\{l_{1}, \ldots, l_{k}\right\}=\left\{i_{0}, \ldots, \widehat{i_{s}}, \ldots, i_{\mu}, K_{\widehat{j_{s_{2}}}}, K_{\widehat{j_{s_{3}}}}, \ldots, K_{\widehat{j_{s_{m}}}}\right\}
$$

for some $0 \leqslant s \leqslant \mu$ and some $1 \leqslant s_{h} \leqslant \lambda_{h}+1$ for $h=2, \ldots, m$. In this case

$$
\begin{aligned}
& \frac{(-1)^{k}}{d_{l_{1}, \ldots, l_{k}} f_{i_{0}, i_{1}, \ldots, i_{k}}} \frac{a_{J}}{a\left(J_{\lambda}, J\right)} S^{(a)}\left(s\left(\mathcal{C}_{J_{\lambda}}(x)\right), F_{l_{1}, \ldots, l_{k}}\right) \\
& \quad=\frac{(-1)^{k}}{d_{l_{1}, \ldots, l_{k}} f_{i_{0}, i_{1}, \ldots, i_{k}}} \frac{a_{J}}{a\left(J_{\lambda}, J\right)}(-1)^{s+\sum_{h=2}^{m} s_{h}} \prod_{q=0}^{\mu} a_{i_{q}} \prod_{h=2}^{m} \prod_{q=1}^{\lambda_{h}+1} a_{j_{q} h} .
\end{aligned}
$$

Consider the right-hand side of (4.26). The potential $Q$ of second kind is the sum $\sum \frac{a_{J}}{a\left(J_{\lambda}, J\right)} Q_{\mathcal{C}_{J_{\lambda}}}$ shown in (3.8), where the sum is over all elementary subarrangements $\mathcal{C}_{J_{\lambda}}(x)$ of $\mathcal{C}(x)$.

Lemma 4.4.3. If the derivative

$$
\frac{\partial^{2 k+1} Q_{\mathcal{C}_{J_{\lambda}}}}{\partial z_{i_{0}} \partial z_{i_{1}} \ldots \partial z_{i_{k}} \partial z_{l_{1}} \ldots \partial z_{l_{k}}}
$$


is nonzero, then $F_{i_{1}, \ldots, i_{k}}$ and $F_{l_{1}, \ldots, l_{k}}$ are distinguished elements of $\mathcal{C}_{J_{\lambda}}$.

Proof. The proof is the same as the proof of Lemma 4.3.3.

Clearly the function $\frac{1}{f_{i_{0}, \ldots, i_{\mu}}(z)}$ multiplied by a constant can be obtained by this differentiation only if $\mathcal{C}_{J_{\lambda}}(x) \in E\left(i_{0}, i_{1}, \ldots, i_{\mu}\right)$. In this case we have

$$
\frac{a_{J}}{a\left(J_{\lambda}, J\right)} Q_{\mathcal{C}_{J_{\lambda}}}=\frac{a_{J}}{a\left(J_{\lambda}, J\right)} \frac{\prod_{q=0}^{\mu} a_{i_{q}}}{(2 \mu) !} \ln \left(f_{\mathcal{C}_{J_{\lambda}, 1}}\right) \frac{\left(f_{\mathcal{C}_{J_{\lambda}, 1}}\right)^{2 \mu}}{\left(\prod_{j=0}^{\mu} d_{i_{0}, \ldots, i_{j}, \ldots, i_{\mu} ; 1}\right)^{2}} \prod_{h=2}^{m} P_{\mathcal{C}_{J_{\lambda}, h}},
$$

see formula (3.6). Derivatives of this summand do not depend on the ordering the elements of the sets $J_{h}$, $h=1, \ldots, m$, and we may assume that the equality (4.31) of ordered sets holds.

By Lemma 4.4.3 we may assume that the equality of ordered sets in (4.32) holds. In that case, the operator $(-1)^{k} \frac{\partial^{2 k+1}}{\partial z_{i_{0}} \ldots \partial z_{i_{k}} \partial z_{i_{0}} \ldots \partial z_{i_{s}} \ldots \partial z_{i_{\mu}} \partial z_{i_{\mu+1}} \ldots \partial z_{l_{k}}}$ applied to the expression in (4.35) gives

$$
\begin{aligned}
& (-1)^{k} \frac{a_{J}}{a\left(J_{\lambda}, J\right)} \prod_{q=0}^{\mu} a_{i_{q}} \prod_{h=2}^{m} \prod_{q=1}^{\lambda_{h}+1} a_{j_{q}^{h}} \\
& \quad \times \frac{(-1)^{s+\sum_{h=2}^{m} s_{h}}}{d_{i_{0}, \ldots, \widehat{i_{s}}, \ldots, i_{\mu} ; 1} \prod_{h=2}^{m} d_{j_{1}^{h}, \ldots, j_{s_{h}}^{h}, \ldots, j_{\lambda_{h}+1}^{h} ; h}} \frac{1}{f_{\mathcal{C}_{J_{\lambda}, 1} \prod_{h=2}^{m} d_{j_{2}^{h}, \ldots, j_{\lambda_{h}+1}^{h} ; h}}} .
\end{aligned}
$$

Lemma 3.3.1 implies that

$$
d_{i_{0}, \ldots, \widehat{i_{s}, \ldots, i_{\mu} ; 1}} \prod_{h=2}^{m} d_{j_{1}^{h}, \ldots, \widehat{j_{s_{h}}^{h}}, \ldots, j_{\lambda_{h}+1}^{h} ; h}=d_{l_{1}, \ldots, l_{k}}, \quad f_{\mathcal{C}_{J_{\lambda}, 1}} \prod_{h=2}^{m} d_{j_{2}^{h}, \ldots, j_{\lambda_{h}+1}^{h} ; h}=f_{i_{0}, \ldots, i_{k}} .
$$

Now (4.36) equals (4.33). This proves Theorem 4.4.1.

Corollary 4.4.4. Let $x \in \mathbb{C}^{n}-\Delta$. Then for any two ordered independent subsets $I=\left\{i_{1}, \ldots, i_{k}\right\}, L=$ $\left\{l_{1}, \ldots, l_{k}\right\} \subset J$ and any $i_{0} \in J$, we have

$$
S^{(a)}\left(K_{i_{0}}(x) v_{i_{1}, \ldots, i_{k}}, v_{l_{1}, \ldots, l_{k}}\right)=d_{i_{1}, \ldots, i_{k}} d_{l_{1}, \ldots, l_{k}} \frac{\partial^{2 k+1} Q}{\partial z_{i_{0}} \partial z_{i_{1}} \ldots \partial z_{i_{k}} \partial z_{l_{1}} \ldots \partial z_{l_{k}}}(x)
$$

Proof. The corollary follows from formula (1.9) and Theorems 1.12.1, 2.4.1. 


\section{CHAPTER 5}

\section{Unit Element for 2 and 3-Dimensional Arrangements}

In Chapters 1-4 we studied the algebra of functions on the critical set of the master function. The unit element is the most important element of that algebra. Any other element of the algebra can be obtained from the unit element by multiplication by $p_{1}, \ldots, p_{n}$. An interesting problem is to give a formula for the unit element in terms of the basis $v_{I} \ldots$ In this chapter we give such a formula for an arrangement of lines in $\mathbb{C}^{2}$ and give partial results on the unit element of an arrangement in $\mathbb{C}^{3}$. Similar to the expression for the potential functions, the expression for the unit element is again a sum of contributions from each individual elementary subarrangement.

Let $I=\{1,2, \ldots, N\}$. Let $X$ be a two-dimensional vector space with coordinates $t_{1}$ and $t_{2}$. For each $i \in I$, let $g_{i}=b_{i}^{1} t_{1}+b_{i}^{2} t_{2}$ be a nonzero linear function on $X$. Let $z_{i} \in \mathbb{C}$ and define $f_{i}\left(t_{1}, t_{2}\right)=g_{i}\left(t_{1}, t_{2}\right)+z_{i}$. The zero sets of the $f_{i}$ give rise to an arrangement of lines in $X$. We call this arrangement $\mathcal{C}$, where the line associated to $f_{i}$ is denoted $H_{i}$. Each line $H_{i}$ has weight $a_{i}$. Define $|a|=\sum_{i \in I} a_{i}$

Denote $U=X-\sum_{i=1}^{N} H_{i}$ as the compliment of $\mathcal{C}$, and define $\mathbb{C}(U)$ to be the algebra of rational functions regular on $U$. Define

$$
\Phi=\sum_{i=1}^{N} a_{i} \log \left(f_{i}\right)
$$

as the master function of $\mathcal{C}$. Let $I_{\Phi}=\left\langle\frac{d \Phi}{d t_{1}}, \frac{d \Phi}{d t_{2}}\right\rangle$ and define $A_{\Phi}=\mathbb{C}(U) / I_{\Phi}$. Let $p_{i}=\left[\frac{a_{i}}{f_{i}}\right] \in A_{\Phi}$ and $d_{i j}=\left|\begin{array}{cc}b_{i}^{1} & b_{i}^{2} \\ b_{j}^{1} & b_{j}^{2}\end{array}\right|$. Note that $\left\{p_{i}\right\}_{i \in I}$ spans $A_{\Phi}$. The relations of $A_{\Phi}$ are $\sum_{j \in J} b_{1}^{j} p_{j}=\sum_{j \in J} b_{2}^{j} p_{j}=0$, which implies that for any $i \in I, \sum_{j \in J} d_{i j} p_{j}=0$. Henceforth, we will refer to this relation as the $d$-relation. Define $\omega_{i j}=d_{i j} p_{i} p_{j}$. The $d$-relation can be written in the language of our $\omega_{i j}$ as $\sum_{j \in I} \omega_{i j}=0$ for any $i \in I$. Similarly, the fact that $d_{i j}$ is a determinant implies $\omega_{i j}=-\omega_{j i}$.

For each $i \in I$, let $s_{i ; 1}$ and $s_{i ; 2}$ be coordinates such that $g_{i}=c_{i ; i}^{1} s_{i ; 1}+0 s_{i ; 2}$ when expressed in these 
coordinates. Also, choose the coordinates so that the change of basis matrix from coordinates $\left\{t_{1}, t_{2}\right\}$ to

coordinates $\left\{s_{i ; 1}, s_{i ; 2}\right\}$ has determinant one. For any $j \in I$ let $\left\{c_{j ; i}^{1}, c_{j ; i}^{2}\right\}$ be the coefficients of $g_{j}$ under the coordinates $\left\{s_{i ; 1}, s_{i ; 2}\right\}$. If the line $H_{j}$ is parallel to the line $H_{i}$ and the line $H_{k}$ is not parallel to the line $H_{i}$, then the determinant $d_{j k}$ decomposes into $d_{j k}=c_{j ; i}^{1} c_{k ; i}^{2}$. In this same case where $H_{j}$ and $H_{k}$ are parallel to and anti-parallel to $H_{i}$, we define

$$
\begin{aligned}
& d_{j ; 1}^{i}=c_{j}^{1} \\
& d_{k ; 2}^{i}=c_{k}^{2}
\end{aligned}
$$

so that we can also decompose the determinant $d_{i j}$ as $d_{j ; 1}^{i} d_{k ; 2}^{i}=d_{j k}$.

\subsection{Formula for the Unit Element of $A_{\Phi}$ in 2-Dimensions}

Reorder $I$ if necessary so that the lines $H_{1}$ and $H_{2}$ (the zero sets of the $f$ function associated with the first two elements of $I$ ) are not parallel.

Define the following functions:

$$
\begin{aligned}
f_{i j k} & =d_{j k} f_{i}-d_{i k} f_{j}+d_{i j} f_{k}=d_{j k} z_{i}-d_{i k} z_{j}+d_{i j} z_{k} \\
f_{i j ; 1} & =d_{j ; 1}^{i} f_{i}-d_{i ; 1}^{i} f_{j}=d_{j ; 1}^{i} z_{i}-d_{i ; 1}^{i} z_{j} \\
f_{i j, k ; 2} & =d_{j ; 2}^{k} f_{i}-d_{i ; 2}^{k} f_{j}-\frac{d_{i j}}{d_{k ; 1}^{k}} f_{k}=d_{j ; 2}^{k} z_{i}-d_{i ; 2}^{k} z_{j}-\frac{d_{i j}}{d_{k ; 1}^{k}} z_{k}
\end{aligned}
$$

Let $\mathcal{C}_{J_{\lambda}}^{1}$ be an elementary arrangement that contains the line $H_{1}$. It may be helpful to review section 3.1 at this time if you are not absolutely certain what this notation means, or if at any time in this section you are not $100 \%$ certain what a certain bit of notation or definition means. As stated in the introduction to this chapter, the unit element (a global property of $A_{\Phi}$ ) will be constructed by contributions from each elementary subarrangement (which are local structures in the arrangement). The contribution of the elementary arrangement $\mathcal{C}_{J_{\lambda}}^{1}$ is designated by $\mathbf{1}_{C_{J_{\lambda}}}^{1}$.

The form of $\mathbf{1}_{C_{J_{\lambda}}}^{1}$ depends on properties of the elementary arrangement $\mathcal{C}_{J_{\lambda}}^{1}$. Specifically, $\mathbf{1}_{C_{J_{\lambda}}}^{1}$ depends on both the type of the elementary arrangement, which was defined as $\lambda$ in Chapter 3, and in which Auxillary 
Arrangement the line $H_{1}$ lies in inside $\mathcal{C}_{J_{\lambda}}^{1}$.

Type (2): For $C_{J_{\lambda}}^{1}$ of type $\lambda=(2)$, with $J_{(2)}=\{i, j, 1\}$ we have

$$
\mathbf{1}_{C_{J_{\lambda}}^{1}}=\frac{1}{|a|^{2}} \frac{-f_{i j 1}^{2}}{d_{i 1} d_{i j} d_{j 1}} \omega_{i j}
$$

Type $(1,1)$ with 1 in first auxillary arrangment: For $C_{J_{\lambda}}^{1}$ of type $(1,1)$ with $J_{1}=\{1, h\}$ and $J_{2}=$ $\{i, 2\}$ we have

$$
\mathbf{1}_{C_{J_{\lambda}}^{1}}=\frac{1}{|a|^{2}} \frac{-f_{h 1 ; 1}}{d_{1 ; 1}^{1} d_{h ; 1}^{1}} \frac{f_{i 2,1 ; 2}+f_{i 2, h ; 2}}{d_{2 ; 2}^{1} d_{i ; 2}^{1}} \omega_{i h}+\frac{a_{1}}{|a|^{2} a(1)} \frac{-f_{h 1 ; 1}}{d_{1 ; 1}^{1} d_{h ; 1}^{1}} \frac{f_{i 2, h ; 2}}{d_{i ; 2}^{1} d_{2 ; 2}^{1}} \omega_{h i}+\frac{a_{h}}{|a|^{2} a(h)} \frac{-f_{1 h ; 1}}{d_{h ; 1}^{h} d_{1 ; 1}^{h}} \frac{f_{i 2,1 ; 2}}{d_{i ; 2}^{h} d_{2 ; 2}^{h}} \omega_{1 i}
$$

Type (1,1) with 1 in second auxillary arrangement: For $C_{J_{\lambda}}^{1}$ of type $(1,1)$ with $J_{1}=\{i, j\}$ and $J_{2}=\{1, k\}$, we have

$$
\mathbf{1}_{C_{J_{\lambda}}^{1}}=\frac{a_{i}}{|a|^{2} a(i)}\left(-\frac{f_{j i ; 1}}{d_{i ; 1}^{i} d_{j ; 1}^{i}}\right) \frac{f_{k 1, j ; 2}}{d_{1 ; 2}^{i} d_{k ; 2}^{i}} \omega_{j k}+\frac{a_{j}}{|a|^{2} a(j)}\left(-\frac{f_{i j ; 1}}{d_{j ; 1}^{j} d_{i ; 1}^{j}}\right) \frac{f_{k 1, i ; 2}}{d_{1 ; 2}^{j} d_{k ; 2}^{j}} \omega_{i k}
$$

Type $(1,1)$ with both auxillary arrangements having two parallel lines: For $C_{J_{\lambda}}^{1}$ of type $(1,1)$ with $J_{1}=\{h, 1\}$ and $J_{2}=\{j, i\}$, with $H_{1} \| H_{h}$ and $H_{i} \| H_{j}$, we have

$$
\begin{aligned}
\mathbf{1}_{C_{J_{\lambda}}^{1}} & =\frac{1}{|a|^{2}}\left(\frac{-f_{h 1 ; 1}}{d_{1 ; 1}^{1} d_{h ; 1}^{1}}\right)\left(\frac{f_{i j, h ; 2}+f_{i j, 1 ; 2}}{d_{i ; 2}^{1} d_{j ; 2}^{1}}\right) \omega_{i h} \\
& +\frac{a_{1}}{|a|^{2} a(1)}\left(-\frac{f_{1 h ; 1}}{d_{1 ; 1}^{1} d_{h ; 1}^{1}}\right)\left(\frac{f_{i j, h ; 2}}{d_{i ; 2}^{1} d_{j ; 2}^{1}}\right) \omega_{h i}+\frac{a_{h}}{|a|^{2} a(h)}\left(-\frac{d_{h 1 ; 1}}{d_{1 ; 1}^{1} d_{h ; 1}^{1}}\right)\left(\frac{f_{i j, 1 ; 2}}{d_{i ; 2}^{1} d_{j ; 2}^{1}}\right) \omega_{1 i} \\
& +\frac{a_{i}}{|a|^{2} a(i)}\left(-\frac{f_{i j ; 1}}{d_{i ; 1}^{i} d_{j ; 1}^{i}}\right)\left(\frac{f_{h 1, j ; 2}}{d_{1 ; 2}^{i} d_{h ; 2}^{i}}\right) \omega_{j h}+\frac{a_{j}}{|a|^{2} a(j)}\left(-\frac{f_{j i ; 1}}{d_{i ; 1}^{j} d_{j ; 1}^{j}}\right) \frac{f_{h 1, i ; 2}}{d_{1 ; 2}^{j} d_{h ; 2}^{j}} \omega_{i h}
\end{aligned}
$$

For $i \in I$, define

$$
a(i)=|a|-\sum_{j \in \|_{i}} a_{j}
$$

For any $i \in I$, let $k_{i} \in I$ be the index of a plane $H_{k_{i}}$ such that $k_{i}$ is the smallest index such that $H_{k_{i}} \nVdash H_{i}$. 
Theorem 5.1.1. For each elementary arrangement $\mathcal{C}_{J_{\lambda}}^{1}$ containing the line line $H_{1}$, let $\mathbf{1}_{C_{J_{\lambda}}^{1}}$ be the local contribution to the unit element of $A_{\Phi}$ from $\mathcal{C}_{J_{\lambda}}^{1}$, as defined above. Then, we can describe the unit element of $A_{\Phi}$ as:

$$
\mathbf{1}=\sum_{\mathcal{C}_{J_{\lambda}}^{1}} \mathbf{1}_{\mathcal{C}_{J_{\lambda}}^{1}}
$$

Alternatively, if for each $i \in I$ we define $k_{i} \in I$ to be the smallest index number such that the line $H_{k_{i}}$ is not parallel to $H_{i}$, and we define

$$
\begin{aligned}
\|_{i} & =\left\{j \in I \mid H_{j} \| H_{i}\right\} \cup\{i\}, \\
\triangle_{i} & =\left\{\{j, \ell\} \mid\left\{H_{i}, H_{j}, H_{\ell}\right\} \text { forms a simplex }\right\}, \\
a(i) & =|a|-\sum_{j \in \|_{i}} a_{j},
\end{aligned}
$$

for each $i \in I$, then we can also describe the unit element of $A_{\Phi}$ via the equation

$$
|a|^{2}=\sum_{1} \frac{-f_{i j 1}^{2}}{d_{i 1} d_{i j} d_{j 1}} \omega_{i j}+\sum_{2} \frac{-f_{h 1 ; 1}}{d_{h ; 1}^{1} d_{1 ; 1}^{1}} \frac{f_{i 2,1 ; 2}+f_{i 2, h ; 2}}{d_{2 ; 2}^{1} d_{i ; 2}^{1}} \omega_{i h}+\sum_{i \in I} \frac{a_{i}}{a(i)} \sum_{\ell \in \|_{i}} \sum_{j \notin \|_{i}} \frac{-f_{\ell i ; 1}}{d_{i ; 1}^{i} d_{\ell ; 1}^{i}} \frac{f_{j k_{i}, \ell ; 2}}{d_{k_{i} ; 2}^{i} d_{j ; 2}^{i}} \omega_{\ell j}
$$

where the first sum is over all $i, j \in I$ with $i<j$ and such that $\left\{H_{1}, H_{i}, H_{j}\right\}$ forms an elementary arrangement, and the second sum is over all $i, h \in I$ such that $H_{h} \| H_{1}$ and $\left\{H_{1}, H_{h}, H_{2}, H_{i}\right\}$ forms an elementary arrangement. 


\subsection{Proof of Theorem}

For $i \in I$, define

$$
\begin{aligned}
\|_{i} & =\left\{j \in I \mid H_{j} \| H_{i}\right\} \cup\{i\} \\
\triangle_{i} & =\left\{\{j, \ell\} \mid\left\{H_{i}, H_{j}, H_{\ell}\right\} \text { forms a simplex }\right\} .
\end{aligned}
$$

Let

$$
B=\left\{\omega_{i j} \mid\{i, j\} \in \triangle_{1}\right\} \cup\left\{\omega_{i h} \mid i \in \bigvee_{1}, i \neq 2, h \in \|_{1}\right\}
$$

be a basis of $A_{\Phi}$ that uses $H_{1}$ as the primary basis line, and $H_{2}$ as the secondary basis line. Recall that $|a|=\sum_{i \in I} z_{i} p_{i}$, and thus

$$
|a|^{2}=\left(\sum_{i \in I} z_{i} p_{i}\right)^{2}
$$

We use the the $d$-relations to write this sum as a linear combination of elements of $B$.

1. Let $\{i, j\} \in \triangle_{1}$. Then, $\omega_{i j} \in B$. The left side of the list below shows which monomials from the expansion of the right-hand side (1) will contribute to $\omega_{i j}$ when (1) is written in the basis $B$. The right side of the list shows the contribution the terms on the left-hand side make to the coefficient of $\omega_{i j}$ after (1) is written in basis $B$.

$$
\begin{aligned}
z_{i}^{2} p_{i}^{2} & \rightarrow \frac{-d_{j 1}}{d_{i 1}} z_{i}^{2} \frac{\omega_{i j}}{d_{i j}} \\
z_{j}^{2} p_{j}^{2} & \rightarrow \frac{-d_{i 1}}{d_{j 1}} z_{j}^{2} \frac{\omega_{i j}}{d_{i j}} \\
z_{1}^{2} p_{1}^{2} & \rightarrow \frac{d_{j i} d_{i j}}{d_{1 i} d_{1 j}} z_{1}^{2} \frac{\omega_{i j}}{d_{i j}} \\
2 z_{i} z_{j} p_{i} p_{j} & \rightarrow 2 z_{i} z_{j} \frac{\omega_{i j}}{d_{i j}} \\
2 z_{j} z_{1} p_{j} p_{1} & \rightarrow-2 \frac{d_{i j}}{d_{1 j}} z_{j} z_{1} \frac{\omega_{i j}}{d_{i j}} \\
2 z_{i} z_{1} p_{i} p_{1} & \rightarrow-2 \frac{d_{j i}}{d_{1 i}} z_{i} z_{1} \frac{\omega_{i j}}{d_{i j}} .
\end{aligned}
$$


Summing these contributions, we obtain

$$
\frac{-\left(d_{j 1} z_{i}\right)^{2}-\left(d_{i 1} z_{j}\right)^{2}-\left(d_{i j} z_{1}\right)^{2}+2 d_{i 1} z_{i} z_{j}+2 d_{i j} d_{i 1} z_{j} z_{1}-2 d_{i j} d_{j 1} z_{i} z_{1}}{d_{i k} d_{i j} d_{j 1}} \omega_{i j}=\frac{-f_{i j 1}^{2}}{d_{i 1} d_{i j} d_{j 1}} \omega_{i j} .
$$

2. Let $i, h \in I$ such that $h \in \|_{1}, i \in \bigvee_{1}, h \neq 1, i \neq 2$. The left side of the list below shows which monomials from the expansion of the right-hand side (1) will contribute to $\omega_{i h}$ when (1) is written in the basis $B$. The right side of the list shows the contribution the terms on the left-hand side make to the coefficient of $\omega_{i h}$ after (1) is written in basis $B$.

$$
\begin{aligned}
z_{1}^{2} p_{1}^{2} & \rightarrow \frac{d_{h ; 1}^{1} d_{i 2}}{d_{1 ; 1}^{1} d_{12}} z_{1}^{2} \frac{\omega_{i h}}{d_{i h}} \\
z_{h}^{2} p_{h}^{2} & \rightarrow-\frac{d_{i 2}}{d_{h 2}} z_{h}^{2} \frac{\omega_{i h}}{d_{i h}} \\
2 z_{i} z_{h} p_{i} p_{h} & \rightarrow 2 z_{i} z_{h} \frac{\omega_{i h}}{d_{i h}} \\
2 z_{i} z_{1} p_{i} p_{1} & \rightarrow 2 \frac{-d_{h ; 1}^{1}}{d_{1 ; 1}^{1}} z_{i} z_{1} \frac{\omega_{i h}}{d_{i h}} \\
2 z_{h} z_{2} p_{h} p_{2} & \rightarrow-2 \frac{d_{i h}}{d_{2 h}} z_{h} z_{2} \frac{\omega_{i h}}{d_{i h}} \\
2 z_{2} z_{1} p_{2} p_{1} & \rightarrow 2 \frac{d_{h ; 1}^{1} d_{i 1}}{d_{1 ; 1}^{1} d_{21}} z_{2} z_{1} \frac{\omega_{i h}}{d_{i h}}
\end{aligned}
$$


Summing these contributions, we obtain

$$
\begin{aligned}
& \frac{d_{h ; 1}^{1} d_{h 2} d_{i 2} z_{1}^{2}-d_{1 ; 1}^{1} d_{12} d_{i 2} z_{h}^{2}+d_{1 ; 1}^{1} d_{h 2} d_{12} 2 z_{i} z_{h}-d_{h ; 1}^{1} d_{h 2} d_{12} z_{i} z_{1}+2 d_{1 ; 1}^{1} d_{12} d_{i h} z_{h} z_{2}-2 d_{h ; 1}^{1} d_{i 1} d_{h 2} z_{1} z_{2}}{d_{1 ; 1}^{1} d_{h 2} d_{12} d_{i h}} \omega_{i h} \\
= & \frac{d_{h 2} d_{h ; 1}^{1} z_{1}\left(d_{i 2} z_{1}-d_{12} 2 z_{i}-2 d_{i 1} z_{2}\right)+d_{12} d_{1 ; 1}^{1} z_{h}\left(-d_{i 2} z_{h}+d_{h 2} 2 z_{i}+2 d_{i h} z_{2}\right)}{d_{1 ; 1}^{1} d_{h 2} d_{12} d_{i h}} \omega_{i h} \\
= & \frac{-d_{h 2} d_{h ; 1}^{1} z_{1}\left(d_{1 ; 1}^{1} d_{2 ; 2}^{1} 2 z_{i}-2 d_{1 ; 1}^{1} d_{i ; 2}^{1} z_{2}-d_{i 2} z_{1}\right)+d_{12} d_{1 ; 1}^{1} z_{h}\left(d_{h ; 1}^{1} d_{2 ; 2}^{1} 2 z_{i}-2 d_{h ; 1}^{1} d_{i ; 2}^{1} z_{2}-d_{i 2} z_{h}\right)}{d_{1 ; 1}^{1} d_{h 2} d_{12} d_{i h}} \omega_{i h} \\
= & \frac{-d_{h 2} d_{1 ; 1}^{1} d_{h ; 1}^{1} z_{1}\left(d_{2 ; 2}^{1} 2 z_{i}-2 d_{i ; 2}^{1} z_{2}-\frac{d_{i 2}}{d_{1 ; 1}^{1}} z_{1}\right)+d_{h 2} d_{1 ; 1}^{1} d_{1 ; 1}^{1} z_{h}\left(d_{2 ; 2}^{1} 2 z_{i}-2 d_{i ; 2}^{1} z_{2}-\frac{d_{i 2}}{d_{h ; 1}^{1}} z_{h}\right)}{d_{1,1} d_{h 2} d_{12} d_{i h}} \omega_{i h} \\
= & \frac{-d_{h ; 1}^{1} z_{1}\left(d_{2 ; 2}^{1} 2 z_{i}-2 d_{i ; 2}^{1} z_{2}-\frac{d_{i 2}}{d_{1 ; 1}} z_{1}-\frac{d_{i 2}}{d_{h ; 1}} z_{h}\right)+d_{1 ; 1}^{1} z_{h}\left(d_{2 ; 2} 2 z_{i}-2 d_{i ; 2}^{1} z_{2}-\frac{d_{i 2}}{d_{1 ; 1}} z_{1}-\frac{d_{i 2}}{d_{h ; 1}} z_{h}\right)}{d_{12} d_{i h}} \omega_{i h} \\
= & \frac{\left(d_{1 ; 1}^{1} z_{h}-d_{h ; 1}^{1} z_{1}\right)\left(\left(d_{2 ; 2}^{1} z_{i}-d_{i ; 2}^{1} z_{2}-\frac{d_{i 2}}{d_{1 ; 1}^{1}} z_{1}\right)+\left(d_{2 ; 2}^{1} z_{i}-d_{i ; 2}^{1} z_{2}-\frac{d_{i 2}}{d_{h ; 1}^{1}} z_{h}\right)\right)}{d_{12} d_{i h}} \omega_{i h} \\
= & \frac{-f_{h 1 ; 1}}{d_{h ; 1}^{1} d_{1 ; 1}^{1}} \frac{f_{i 2,1 ; 2}+f_{i 2, h ; 2}}{d_{2 ; 2}^{1} d_{i ; 2}^{1}} \omega_{i h}
\end{aligned}
$$

3. Let $i, j \in I$ such that $i<j$, and $j \in \|_{i}$. Observe that from the sum in (1), we obtain the following contributions to the coefficient of $p_{i} p_{j}$ :

$$
\begin{aligned}
z_{i}^{2} p_{i}^{2} & \rightarrow \frac{-d_{j ; 1}^{i}}{d_{i ; 1}^{i}} z_{i}^{2} p_{i} p_{j} \\
2 z_{i} z_{j} p_{i} p_{j} & \rightarrow 2 z_{i} z_{j} p_{i} p_{j} \\
z_{j}^{2} p_{j}^{2} & \rightarrow \frac{-d_{i ; 1}^{i}}{d_{j ; 1}^{i}} z_{j}^{2} p_{i} p_{j}
\end{aligned}
$$

Summing these contributions, we obtain

$$
\frac{\left(-\left(d_{j ; 1}^{i}\right)^{2} z_{i}^{2}+2 d_{i ; 1}^{i} d_{j ; 1}^{i} z_{i} z_{j}-\left(d_{i ; 1}^{i}\right)^{2} z_{j}^{2}\right)}{d_{i ; 1}^{i} d_{j ; 1}^{i}} p_{i} p_{j}=-\frac{\left(d_{j ; 1}^{i} z_{i}-d_{i ; 1}^{i} z_{j}\right)^{2}}{d_{i ; 1}^{i} d_{j ; 1}^{i}} p_{i} p_{j} .
$$

Thus, the terms corresponding to all pairs $i, j \in I$ with $i<j$ and $j \in \|_{i}$ are

$$
\sum_{\substack{i<j \\ j \in \|_{i}}}-\frac{\left(d_{j ; 1}^{i} z_{i}-d_{i ; 1}^{i} z_{j}\right)^{2}}{d_{i ; 1}^{i} d_{j ; 1}^{i}} p_{i} p_{j}
$$


Instead of directly writing this in the basis $B$

$$
\sum_{\substack{i<j \\ j \in \|_{i}}}-\frac{1}{a(i)} \frac{\left(d_{j ; 1}^{i} z_{i}-d_{i ; 1}^{i} z_{j}\right)^{2}}{d_{i ; 1}^{i} d_{j ; 1}^{i}} p_{i} p_{j}
$$

in the basis $B$. For ease of future computation, we define

$$
\mathcal{Z}_{i}=\sum_{j \in \|_{i}} \frac{a_{j}}{a(i)} \frac{\left(d_{j ; 1}^{i} z_{i}-d_{i ; 1}^{i} z_{j}\right)}{d_{j ; 1}^{i}}
$$

Recall that $\left(d_{j ; 1}^{i} z_{i}-d_{i ; 1}^{i} z_{j}\right) p_{i} p_{j}=a_{i} d_{j ; 1}^{i} p_{j}-a_{j} d_{i ; 1}^{i} p_{i}$. Using this transformation on (2), we obtain

$$
\begin{aligned}
\sum_{\substack{i<j \\
j \in \|_{i}}}-\frac{1}{a(i)} \frac{\left(d_{j ; 1}^{i} z_{i}-d_{i ; 1}^{i} z_{j}\right)^{2}}{d_{i ; 1}^{i} d_{j ; 1}^{i}} p_{i} p_{j} & =\sum_{\substack{i<j \\
j \in \|_{i}}} \frac{1}{a(i)} \frac{\left(d_{i ; 1}^{i} z_{j}-d_{j ; 1}^{i} z_{i}\right)\left(a_{i} d_{j ; 1}^{i} p_{j}-a_{j} d_{i ; 1}^{i} p_{i}\right)}{d_{i ; 1}^{i} d_{j ; 1}^{i}} \\
& =\sum_{i \in I} \sum_{j \in \|_{i}} \frac{a_{j}}{a(i)} \frac{\left(d_{j ; 1}^{i} z_{i}-d_{i ; 1}^{i} z_{j}\right)}{d_{j ; 1}^{i}} p_{i} \\
& =\sum_{i \in I} \mathcal{Z}_{i} p_{i} .
\end{aligned}
$$

Recall that $\frac{1}{|a|} \sum_{i \in I} z_{i} p_{i}=\mathbf{1}$, so we can write $\sum_{i \in I} \mathcal{Z}_{i} p_{i}$ as

$$
\frac{1}{|a|}\left(\sum_{i \in I} z_{i} p_{i}\right)\left(\sum_{i \in I} \mathcal{Z}_{i} p_{i}\right) .
$$

We write (3) in the basis B:

(a) Let $\{i, j\} \in \triangle_{1}$, so $\omega_{i j} \in B$. Note that although the coefficients are different in (2) than in (1), the same terms will contribute to $\omega_{i j}$ in (3) as contributed to $\omega_{i j}$ in (1). We obtain the following 
contributions to the coefficient of $\omega_{i j}$ :

$$
\begin{aligned}
z_{i} \mathcal{Z}_{i} p_{i}^{2} & \rightarrow \frac{-d_{j 1}}{d_{i 1}} z_{i} \mathcal{Z}_{i} \frac{\omega_{i j}}{d_{i j}} \\
z_{j} \mathcal{Z}_{j} p_{j}^{2} & \rightarrow \frac{-d_{i 1}}{d_{j 1}} z_{j} \mathcal{Z}_{j} \frac{\omega_{i j}}{d_{i j}} \\
z_{1} \mathcal{Z}_{1} p_{1}^{2} & \rightarrow \frac{d_{j i} d_{i j}}{d_{1 i} d_{1 j}} z_{1} \mathcal{Z}_{1} \frac{\omega_{i j}}{d_{i j}} \\
\left(z_{i} \mathcal{Z}_{j}+z_{j} \mathcal{Z}_{i}\right) p_{i} p_{j} & \rightarrow\left(z_{i} \mathcal{Z}_{j}+z_{j} \mathcal{Z}_{i}\right) \frac{\omega_{i j}}{d_{i j}} \\
\left(z_{j} \mathcal{Z}_{1}+z_{1} \mathcal{Z}_{j}\right) p_{j} p_{1} & \rightarrow-\frac{d_{i j}}{d_{1 j}}\left(z_{j} \mathcal{Z}_{1}+z_{1} \mathcal{Z}_{j}\right) \frac{\omega_{i j}}{d_{i j}} \\
\left(z_{i} \mathcal{Z}_{1}+z_{1} \mathcal{Z}_{i}\right) p_{i} p_{k} & \rightarrow-\frac{d_{j i}}{d_{1 i}}\left(z_{i} \mathcal{Z}_{1}+z_{1} \mathcal{Z}_{i}\right) \frac{\omega_{i j}}{d_{i j}}
\end{aligned}
$$

Summing these contributions in a similar fashion as in part 1 , we obtain

$$
\frac{1}{|a|} \frac{-f_{i j 1} \mathcal{F}_{i j 1}}{d_{i 1} d_{i j} d_{j 1}} \omega_{i j}
$$

where the $\mathcal{F}$ function is defined the same as the $f$ function, just with $\mathcal{Z}$ instead of $z$. For ease of future computation, denote

$$
c_{i j}=\frac{-f_{i j 1} \mathcal{F}_{i j 1}}{d_{i 1} d_{i j} d_{j 1}}
$$

(b) Let $i, h \in I$ such that $h \in \|_{k}, i \in \bigvee_{k}$, and $i \neq y$, so $\omega_{i h} \in B$. Again, although the coefficients are different in (3) than in (1), the same terms contribute to $\omega_{i h}$. We obtain the following contributions to the coefficient of $\omega_{i h}$ :

$$
\begin{aligned}
z_{1} \mathcal{Z}_{1} p_{1}^{2} & \rightarrow \frac{d_{h ; 1}^{1} d_{i 2}}{d_{1 ; 1}^{1} d_{12}} z_{1} \mathcal{Z}_{1} \frac{\omega_{i h}}{d_{i h}} \\
z_{h} \mathcal{Z}_{h} p_{h}^{2} & \rightarrow-\frac{d_{i 2}}{d_{h 2}} z_{h} \mathcal{Z}_{h} \frac{\omega_{i h}}{d_{i h}} \\
\left(z_{i} \mathcal{Z}_{h}+z_{h} \mathcal{Z}_{i}\right) p_{i} p_{h} & \rightarrow\left(z_{i} \mathcal{Z}_{h}+z_{h} \mathcal{Z}_{i}\right) \frac{\omega_{i h}}{d_{i h}} \\
\left(z_{i} \mathcal{Z}_{1}+z_{1} \mathcal{Z}_{i}\right) p_{i} p_{1} & \rightarrow \frac{-d_{h ; 1}^{1}}{d_{1 ; 1}^{1}}\left(z_{i} \mathcal{Z}_{1}+z_{1} \mathcal{Z}_{i}\right) \frac{\omega_{i h}}{d_{i h}} \\
\left(z_{h} \mathcal{Z}_{2}+z_{2} \mathcal{Z}_{h}\right) p_{h} p_{2} & \rightarrow-\frac{d_{i h}}{d_{2 h}}\left(z_{h} \mathcal{Z}_{2}+z_{2} \mathcal{Z}_{h}\right) \frac{\omega_{i h}}{d_{i h}} \\
\left(z_{2} \mathcal{Z}_{1}+z_{1} \mathcal{Z}_{2}\right) p_{2} p_{1} & \rightarrow \frac{d_{h ; 1}^{1} d_{i 1}}{d_{1 ; 1}^{1} d_{21}}\left(z_{2} \mathcal{Z}_{1}+z_{1} \mathcal{Z}_{2}\right) \frac{\omega_{i h}}{d_{i h}}
\end{aligned}
$$


Summing these contributions in a similar fashion as in part 2, we obtain

$$
\frac{1}{|a|}\left(\frac{-\mathcal{F}_{h 1 ; 1}}{d_{h ; 1}^{1} d_{1 ; 1}^{1}} \frac{f_{i 2,1 ; 2}}{d_{2 ; 2}^{1} d_{i ; 2}^{1}}+\frac{-f_{h 1 ; 1}}{d_{h ; 1}^{1} d_{1 ; 1}^{1}} \frac{\mathcal{F}_{i 2, h ; 2}}{d_{2 ; 2}^{1} d_{i ; 2}^{1}}\right) \omega_{i h}
$$

where the $\mathcal{F}$ functions are defined the same as the $f$ functions, but with $\mathcal{Z}$ instead of $z$. For ease of future computation, denote

$$
c_{i h}=\frac{-\mathcal{F}_{h 1 ; 1}}{d_{h ; 1}^{1} d_{1 ; 1}^{1}} \frac{f_{i 2,1 ; 2}}{d_{2 ; 2}^{1} d_{i ; 2}^{1}}+\frac{-f_{h 1 ; 1}}{d_{h ; 1}^{1} d_{1 ; 1}^{1}} \frac{\mathcal{F}_{i 2, h ; 2}}{d_{2 ; 2}^{1} d_{i ; 2}^{1}} .
$$

(c) Let $i, j \in I$ such that $i<j$, and $j \in \|_{i}$. We obtain the following contributions to the coefficient of $p_{i} p_{j}$ from (3):

$$
\begin{aligned}
z_{i} \mathcal{Z}_{i} p_{i}^{2} & \rightarrow \frac{-d_{j ; 1}^{i}}{d_{i ; 1}^{i}} z_{i} \mathcal{Z}_{i} p_{i} p_{j} \\
\left(z_{i} \mathcal{Z}_{j}+z_{j} \mathcal{Z}_{i}\right) p_{i} p_{j} & \rightarrow\left(z_{i} \mathcal{Z}_{j}+z_{j} \mathcal{Z}_{i}\right) p_{i} p_{j} \\
z_{j} \mathcal{Z}_{j} p_{j}^{2} & \rightarrow \frac{-d_{i ; 1}^{i}}{d_{j ; 1}^{i}} z_{j} \mathcal{Z}_{j} p_{i} p_{j}
\end{aligned}
$$

Summing these contributions, we obtain

$$
\frac{\left(-\left(d_{j ; 1}^{i}\right)^{2} z_{i} \mathcal{Z}_{i}+d_{i ; 1}^{i} d_{j ; 1}^{i} z_{i} \mathcal{Z}_{j}+d_{i ; 1}^{i} d_{j ; 1}^{i} z_{j} \mathcal{Z}_{i}-\left(d_{i ; 1}^{i}\right)^{2} z_{j} \mathcal{Z}_{j}\right)}{d_{i ; 1}^{i} d_{j ; 1}^{i}} p_{i} p_{j} .
$$




\section{Observe that}

$$
\begin{aligned}
& -\left(d_{j ; 1}^{i}\right)^{2} z_{i} \mathcal{Z}_{i}+d_{i ; 1}^{i} d_{j ; 1}^{i} z_{i} \mathcal{Z}_{j}+d_{i ; 1}^{i} d_{j ; 1}^{i} z_{j} \mathcal{Z}_{i}-\left(d_{i ; 1}^{i}\right)^{2} z_{j} \mathcal{Z}_{j} \\
= & \left(d_{i ; 1}^{i} z_{j}-d_{j ; 1}^{i} z_{i}\right) d_{j ; 1}^{i} \mathcal{Z}_{i}+\left(d_{j ; 1}^{i} z_{i}-d_{i ; 1}^{i} z_{j}\right) d_{i ; 1}^{i} \mathcal{Z}_{j} \\
= & \left(d_{j ; 1}^{i} z_{i}-d_{i ; 1}^{i} z_{j}\right)\left(d_{i ; 1}^{i} \mathcal{Z}_{j}-d_{j ; 1}^{i} \mathcal{Z}_{i}\right) \\
= & \left(d_{j ; 1}^{i} z_{i}-d_{i ; 1}^{i} z_{j}\right)\left(d_{i ; 1}^{i} \sum_{\ell \in \|_{j}} \frac{a_{\ell}}{a(j)} \frac{\left(d_{\ell ; 1}^{j} z_{j}-d_{j ; 1}^{j} z_{\ell}\right)}{d_{\ell ; 1}^{j}}-d_{j ; 1}^{i} \sum_{\ell \in \|_{i}} \frac{a_{\ell}}{a(i)} \frac{\left(d_{\ell ; 1}^{i} z_{i}-d_{i ; 1}^{i} z_{\ell}\right)}{d_{\ell ; 1}^{i}}\right) \\
= & \left(d_{j ; 1}^{i} z_{i}-d_{i ; 1}^{i} z_{j}\right)\left(\sum_{\ell \in i} \frac{a_{\ell}}{a(i)}\left(\frac{d_{i ; 1}^{i}\left(d_{\ell ; 1}^{j} z_{j}-d_{j ; 1}^{j} z_{\ell}\right)-d_{j ; 1}^{i}\left(d_{\ell ; 1}^{i} z_{i}-d_{i ; 1}^{i} z_{\ell}\right)}{d_{\ell ; 1}^{i}}\right)\right) \\
= & \left(d_{j ; 1}^{i} z_{i}-d_{i ; 1}^{i} z_{j}\right)\left(\sum_{\ell \in i} \frac{a_{\ell}}{a(i)}\left(\frac{d_{i ; 1}^{i} d_{\ell ; 1}^{j} z_{j}-d_{i ; 1}^{i} d_{j ; 1}^{j} z_{\ell}-d_{j ; 1}^{i} d_{\ell ; 1}^{i} z_{i}+d_{j ; 1}^{i} d_{i ; 1}^{i} z_{\ell}}{d_{\ell ; 1}^{i}}\right)\right) \\
= & \left(d_{j ; 1}^{i} z_{i}-d_{i ; 1}^{i} z_{j}\right)\left(\sum_{\ell \in i} \frac{a_{\ell}}{a(i)}\right)\left(d_{i ; 1}^{i} z_{j}-d_{j ; 1}^{i} z_{i}\right) \\
= & -\left(\sum_{\ell \in i} \frac{a_{\ell}}{a(i)}\right)\left(d_{j ; 1}^{i} z_{i}-d_{i ; 1}^{i} z_{j}\right)^{2}
\end{aligned}
$$

so the $p_{i} p_{j}$ terms in (3) are:

$$
-\left(\frac{\sum_{\ell \in \|_{i}} a_{\ell}}{a(i)}\right) \frac{\left(d_{j ; 1}^{i} z_{i}-d_{i ; 1}^{i} z_{j}\right)^{2}}{d_{i ; 1}^{i} d_{j ; 1}^{i}} p_{i} p_{j} .
$$


(d) Summing the results of parts (a), (b), and (c) gives us

$$
\begin{aligned}
& \sum_{\substack{i<j \\
j \in \|_{i}}}-\frac{1}{a(i)} \frac{\left(d_{j ; 1}^{i} z_{i}-d_{i ; 1}^{i} z_{j}\right)^{2}}{d_{i ; 1}^{i} d_{j ; 1}^{i}} p_{i} p_{j}=\sum_{\{i, j\} \in \triangle_{1}} \frac{1}{|a|} c_{i j} \omega_{i j}+\sum_{\substack{i \neq 2 \\
(i, h) \in \mathbb{W}_{1} \otimes \|_{1}}} \frac{1}{|a|} c_{i h} \omega_{i h} \\
& +\sum_{\substack{i<j \\
j \in \|_{i}}} \frac{-\sum_{\ell \in \|_{i}} a_{\ell}}{|a| a(i)} \frac{\left(d_{j, ; 1}^{i} z_{i}-d_{i ; 1}^{i} z_{j}\right)^{2}}{d_{i ; 1}^{i} d_{j ; 1}^{i}} p_{i} p_{j} \\
& \sum_{\substack{i<j \\
j \in \|_{i}}} \frac{-\left(|a|-\sum_{\left.\ell \in \|_{i} a_{\ell}\right)}\right.}{|a| a(i)} \frac{\left(d_{j ; 1}^{i} z_{i}-d_{i ; 1}^{i} z_{j}\right)^{2}}{d_{i ; 1}^{i} d_{j ; 1}^{i}} p_{i} p_{j}=\sum_{\{i, j\} \in \triangle_{1}} \frac{1}{|a|} c_{i j} \omega_{i j}+\sum_{\substack{i \neq 2 \\
(i, h) \in \bigvee_{1} \otimes \|_{1}}} \frac{1}{|a|} c_{i h} \omega_{i h} \\
& \sum_{\substack{i<j \\
j \in \|_{i}}}-\frac{a(i)}{|a| a(i)} \frac{\left(d_{j ; 1}^{i} z_{i}-d_{i ; 1}^{i} z_{j}\right)^{2}}{d_{i ; 1}^{i} d_{j ; 1}^{i}} p_{i} p_{j}=\sum_{\{i, j\} \in \triangle_{1}} \frac{1}{|a|} c_{i j} \omega_{i j}+\sum_{\substack{i \neq 2 \\
(i, h) \in \bigvee_{1} \otimes \|_{1}}} \frac{1}{|a|} c_{i h} \omega_{i h} \\
& \frac{1}{|a|} \sum_{\substack{i<j \\
j \in \|_{i}}}-\frac{\left(d_{j ; 1}^{i} z_{i}-d_{i ; 1}^{i} z_{j}\right)^{2}}{d_{i ; 1}^{i} d_{j ; 1}^{i}} p_{i} p_{j}=\sum_{\substack{\{i, j\} \in \triangle_{1} \\
|a|}} \frac{1}{\mid j} \omega_{i j}+\sum_{\substack{i \neq 2 \\
(i, h) \in \nVdash_{1} \otimes \|_{1}}} \frac{1}{|a|} c_{i h} \omega_{i h} \\
& \sum_{\substack{i<j \\
j \in \|_{i}}}-\frac{\left(d_{j ; 1}^{i} z_{i}-d_{i ; 1}^{i} z_{j}\right)^{2}}{d_{i ; 1}^{i} d_{j ; 1}^{i}} p_{i} p_{j}=\sum_{\{i, j\} \in \triangle_{1}} c_{i j} \omega_{i j}+\sum_{\substack{i \neq 2 \\
(i, h) \in \bigvee_{1} \otimes \|_{1}}} c_{i h} \omega_{i h} .
\end{aligned}
$$

So, the contributions from the $p_{i} p_{j}$ terms are:

$$
\sum_{\{i, j\} \in \triangle_{1}} \frac{-f_{i j 1} \mathcal{F}_{i j 1}}{d_{i 1} d_{i j} d_{j 1}} \omega_{i j}+\sum_{\substack{i \neq 2 \\(i, h) \in \bigvee_{1} \otimes \|_{1}}}\left(\frac{-\mathcal{F}_{h 1 ; 1}}{d_{h ; 1}^{1} d_{1 ; 1}^{1}} \frac{f_{i 2,1 ; 2}}{d_{2 ; 2}^{1} d_{i ; 2}^{1}}+\frac{-f_{h 1 ; 1}}{d_{h ; 1}^{1} d_{1 ; 1}^{1}} \frac{\mathcal{F}_{i 2, h ; 2}}{d_{2 ; 2}^{1} d_{i ; 2}^{1}}\right) \omega_{i h} .
$$

Combining the results of parts 1,2 , and 3 gives

$$
\begin{aligned}
|a|^{2} & =\sum_{1} \frac{-f_{i j 1}^{2}}{d_{i 1} d_{i j} d_{j 1}} \omega_{i j}+\sum_{2}\left(\frac{-f_{h 1 ; 1}}{d_{h ; 1}^{1} d_{1 ; 1}^{1}} \frac{f_{i 2,1 ; 2}}{d_{2 ; 2}^{1} d_{i ; 1}^{1}}+\frac{-f_{h 1 ; 1}}{d_{h ; 1}^{1} d_{1 ; 1}^{1}} \frac{f_{i 2, h ; 1}}{d_{2 ; 2}^{1} d_{i ; 1}^{1}}\right) \omega_{i h} \\
& +\sum_{1} \frac{-f_{i j 1} \mathcal{F}_{i j 1}}{d_{i 1} d_{i j} d_{j 1}} \omega_{i j}+\sum_{2}\left(\frac{-\mathcal{F}_{h 1 ; 1}}{d_{h ; 1}^{1} d_{1 ; 1}^{1}} \frac{f_{i 2,1 ; 2}}{d_{2 ; 2}^{1} d_{i ; 2}^{1}}+\frac{-f_{h 1 ; 1}}{d_{h ; 1}^{1} d_{1 ; 1}^{1}} \frac{\mathcal{F}_{i 2, h ; 2}}{d_{2 ; 2}^{1} d_{i ; 2}^{1}}\right) \omega_{i h}
\end{aligned}
$$

where the first sum is over all $i, j \in I$ with $i<j$ and such that $\left\{H_{1}, H_{i}, H_{j}\right\}$ forms an elementary arrangement, and the second sum is over all $i, h \in I$ such that $H_{h} \| H_{1}$ and $\left\{H_{1}, H_{h}, H_{2}, H_{i}\right\}$ forms an elementary arrangement. This is (1) written in the basis $B$. 
Recall that there are weighted terms inside each of the $\mathcal{Z}_{i}$. The only place where such weighted terms apear is on the second line of (4). Let $i \in I$ such that $i \notin\left\|_{1} \cup\right\|_{2}$. We can show that once formula 4 is expanded, the term associated with weight $\frac{a_{i}}{a(i)}$ is

$$
\left(\sum_{\substack{j \neq i \\ j \in \|_{t}}} \sum_{\ell \neq \|_{t}} \frac{f_{j i ; 1}}{d_{j ; 1}^{i} d_{i ; 1}^{i}} \frac{f_{\ell 1, j ; 2}}{d_{1 ; 2}^{i} d_{\ell ; 2}^{i}} w_{j \ell}\right) \frac{a_{i}}{a(i)}
$$

This simple coefficient for $\frac{a_{t}}{a(t)}$ is due to the fact that $i$ is not associated with the basis that our formula for $|a|^{2}$ is written in. If we were attempt to write out the term associated with $\frac{a_{1}}{a(1)}, \frac{a_{2}}{a(2)}$, or even $\frac{a_{h}}{a(h)}, \frac{a_{x}}{a(x)}$ for $h \in\left\|_{1}, x \in\right\|_{2}$ the coefficient would be far more complex. Note that we can avoid this complexity through the following argument.

Say that we developed a new formula similar to (4), but in a basis where $H_{1}$ and $H_{2}$ were not basis lines (or even parallel to basis lines). Following the same construction that led us (4), the terms associated with $\frac{a_{1}}{a(1)}$ and $\frac{a_{2}}{a(2)}$ (and $\frac{a_{h}}{a(h)}$ and $\frac{a_{x}}{a(x)}$ ) would be as simple as (5). Since both formula (4) and the new formula are ways of expressing $|a|^{2}$, they must be themselves equal. Each of them have weighted terms of the form $\frac{a_{i}}{a(i)}$ for all $i \in I$. Since these weights are distinct, the coefficients associated with these weights must be equal in both formulas. Thus, the overly complex coefficent for $\frac{a_{1}}{a(1)}$ that would result from trying to express the coefficient in the basis $B$ can instead be replaced by the simpler coefficient found using the new formula.

With this argument, we reason that if we define $k_{i}, \ell_{i} \in I$ to be the smallest indices such that $\left\{H_{k_{i}} H_{\ell_{i}}, H_{i}\right\}$ form a simplex, then we can express the coefficient for $\frac{a_{i}}{a(i)}$ in the basis where $H_{k_{i}}$ and $H_{\ell_{i}}$ are the first and second basis line as

$$
\frac{a_{i}}{a(i)} \sum_{\ell \in \|_{i}} \sum_{j \notin \|_{i}} \frac{-f_{\ell i ; 1}}{d_{i ; 1}^{i} d_{\ell ; 1}^{i}} \frac{f_{j k_{i}, \ell ; 2}}{d_{k_{i} ; 2}^{i} d_{j ; 2}^{i}} \omega_{\ell j} .
$$

Since the second line of (4) is the only place where such weighted terms appear, and moreover the second line consists of only weighted terms, we have shown that

$$
\sum_{1} \frac{-f_{i j 1} \mathcal{F}_{i j 1}}{d_{i 1} d_{i j} d_{j 1}} \omega_{i j}+\sum_{2}\left(\frac{-\mathcal{F}_{h 1 ; 1}}{d_{h ; 1}^{1} d_{1 ; 1}^{1}} \frac{f_{i 2,1 ; 2}}{d_{2 ; 2}^{1} d_{i ; 2}^{1}}+\frac{-f_{h 1 ; 1}}{d_{h ; 1}^{1} d_{1 ; 1}^{1}} \frac{\mathcal{F}_{i 2, h ; 2}}{d_{2 ; 2}^{1} d_{i ; 2}^{1}}\right) \omega_{i h}=\frac{a_{i}}{a(i)} \sum_{\ell \in \|_{i}} \sum_{j \notin \|_{i}} \frac{-f_{\ell i ; 1}}{d_{i ; 1}^{i} d_{\ell ; 1}^{i}} \frac{f_{j k_{i}, \ell ; 2}}{d_{k_{i} ; 2}^{i} d_{j ; 2}^{i}} \omega_{\ell j}
$$


and thus

$$
|a|^{2}=\sum_{1} \frac{-f_{i j 1}^{2}}{d_{i 1} d_{i j} d_{j 1}} \omega_{i j}+\sum_{2} \frac{-f_{h 1 ; 1}}{d_{h ; 1}^{1} d_{1 ; 1}^{1}} \frac{f_{i 2,1 ; 2}+f_{i 2, h ; 2}}{d_{2 ; 2}^{1} d_{i ; 2}^{1}} \omega_{i h}+\sum_{i \in I} \frac{a_{i}}{a(i)} \sum_{\ell \in \|_{i}} \sum_{j \notin \|_{i}} \frac{-f_{\ell i ; 1}}{d_{i ; 1}^{i} d_{\ell ; 1}^{i}} \frac{f_{j k_{i}, \ell ; 2}}{d_{k_{i} ; 2}^{i} d_{j ; 2}^{i}} \omega_{\ell j}
$$

where the first sum is over all $i, j \in I$ with $i<j$ and such that $\left\{H_{1}, H_{i}, H_{j}\right\}$ forms an elementary arrangement, and the second sum is over all $i, h \in I$ such that $H_{h} \| H_{1}$ and $\left\{H_{1}, H_{h}, H_{2}, H_{i}\right\}$ forms an elementary arrangement.

Note that this formula is equivalent to

$$
\mathbf{1}=\sum_{\mathcal{C}_{J_{\lambda}}^{1}} \mathbf{1}_{\mathcal{C}_{J_{\lambda}}^{1}}
$$

\subsection{Conjectural Formula for 3-Dimensional Unit Element}

Let $I=\{1,2, \ldots, N\}$. Let $X$ be a three-dimensional vector space with coordinates $t_{1}, t_{2}$, and $t_{3}$. For each $i \in I$, let $g_{i}=b_{i}^{1} t_{1}+b_{i}^{2} t_{2}+b_{i}^{3} t_{3}$ be a nonzero linear function on $X$. Let $z_{i} \in \mathbb{C}$ and define $f_{i}\left(t_{1}, t_{2}, t_{3}\right)=g_{i}\left(t_{1}, t_{2}, t_{3}\right)+z_{i}$. The zero sets of the $f_{i}$ give rise to an arrangement of planes in $X$. We call this arrangement $\mathcal{C}$, where the plane associated to $f_{i}$ is denoted $H_{i}$. Each line $H_{i}$ has weight $a_{i}$. Define $|a|=\sum_{i \in I} a_{i}$

Denote $U=X-\sum_{i=1}^{N} H_{i}$ as the compliment of $\mathcal{C}$, and define $\mathbb{C}(U)$ to be the algebra of rational functions regular on $U$. Define

$$
\Phi=\sum_{i=1}^{N} a_{i} \log \left(f_{i}\right)
$$

as the master function of $\mathcal{C}$. Let $I_{\Phi}=\left\langle\frac{d \Phi}{d t_{1}}, \frac{d \Phi}{d t_{2}}, \frac{d \Phi}{d t_{3}}\right\rangle$ and define $A_{\Phi}=\mathbb{C}(U) / I_{\Phi}$. Let $p_{i}=\left[\frac{a_{i}}{f_{i}}\right] \in A_{\Phi}$ and $d_{i j k}=\left|\begin{array}{rrr}b_{i}^{1} & b_{i}^{2} & b_{i}^{3} \\ b_{j}^{1} & b_{j}^{2} & b_{j}^{3} \\ b_{k}^{1} & b_{k}^{2} & b_{k}^{3}\end{array}\right|$. Note that $\left\{p_{i}\right\}_{i \in I}$ spans $A_{\Phi}$. The relations on $A_{\Phi}$, are $\sum_{j \in J} b_{1}^{j} p_{j}=\sum_{j \in J} b_{2}^{j} p_{j}=$ $\sum_{j \in J} b_{3}^{j} p_{j}=0$, which implies that for any pair $i, j \in I, \sum_{k \in J} d_{i j k} p_{k}=0$. We will refer to this relation as 
the $d$-relation. Define $\omega_{i j k}=d_{i j k} p_{i} p_{j} p_{k}$. Observe that due to the properties of the determinant $d_{i j k}$, we have $\omega_{i j k}=(-1)^{|\sigma|} \omega_{\sigma(i) \sigma(j) \sigma(k)}$ for any cyclic permutation $\sigma$ and $\sum_{k \in I} \omega_{i j k}=0$ for any pair $i, j \in I$.

For $i \in I$, define

$$
a(i)=|a|-\sum a_{j}
$$

Where the sum is over all indices such that $d_{i j k}=0$ but

Define the following functions:

$$
\begin{aligned}
f_{i j k \ell} & =d_{j k \ell} f_{i}-d_{i k \ell} f_{j}+d_{i j \ell} f_{k}-d_{i j k} f_{\ell}=d_{j k \ell} z_{i}-d_{i k \ell} z_{j}+d_{i j \ell} z_{k}-d_{i j k} z_{\ell} \\
f_{i j ; 1} & =d_{j ; 1}^{i} f_{i}-d_{i ; 1}^{i} f_{j}=d_{j ; 1}^{i} z_{i}-d_{i ; 1}^{i} z_{j} \\
f_{k \ell, i j ; 2} & =d_{\ell ; 2}^{i} f_{k}-d_{k ; 2}^{i} f_{\ell}+\frac{d_{k \ell j}}{d_{i j ; 1}^{i}} f_{i}+\frac{d_{i \ell k}}{d_{j i ; 1}^{i}} f_{j}=d_{\ell ; 2}^{i} z_{k}-d_{k ; 2}^{i} z_{\ell}+\frac{d_{k \ell j}}{d_{i j ; 1}^{i}} z_{i}+\frac{d_{i \ell k}}{d_{j i ; 1}^{i}} z_{j} \\
f_{j k l, i ; 2} & =d_{k \ell ; 2}^{i} f_{j}-d_{j \ell ; 2}^{i} f_{k}+d_{j k ; 2}^{i} f_{\ell}-\frac{d_{j k \ell}}{d_{i ; 1}^{i}} f_{i}=d_{k \ell ; 2}^{i} z_{j}-d_{j \ell ; 2}^{i} z_{k}+d_{j k ; 2}^{i} z_{\ell}-\frac{d_{j k \ell}}{d_{i ; 1}^{i}} z_{i}
\end{aligned}
$$

Order $I$ such that $d_{123} \neq 0$.

Let $\mathcal{C}_{J_{\lambda}}^{1}$ be an elementary arrangement that contains the plane $H_{1}$. We would think that the unit element of $A_{\Phi}$ in the 3-dimensional case would closely mirror the composition of the 2-dimensional unit element. So, we expect to find contributions from each type of arrangement. Below we list these. The ones that are known are marked fact, and the ones that are suspected but not proved are marked conjecture.

As in the 2-dimensional case, if we let $\mathcal{C}_{J_{\lambda}}^{1}$ be an elementary arrangement containing the plane $H_{1}$, then we define $\mathbf{1}_{\mathcal{C}_{J_{\lambda}}^{1}}$ to be the contribution to the unit element of $A_{\Phi}$ from $\mathcal{C}_{J_{\lambda}}^{1}$. We expect that the form of this contribution depends on the type $\lambda$ of the elementary arrangement.

Type (3): (Fact) For $\mathcal{C}_{J_{\lambda}}$ of type $\lambda=(2)$, with $J_{(3)}=\{i, j, k, 1\}$ we have

$$
\mathbf{1}_{\mathcal{C}_{J_{\lambda}}^{1}}=\frac{1}{|a|^{3}} \frac{-f_{i j k 1}^{3}}{d_{j k 1} d_{i k 1} d_{i j 1} d_{i j k}} \omega_{i j k}
$$


Type $(2,1)$ with 1 in first auxillary arrangment: (Fact) For $\mathcal{C}_{J_{\lambda}}$ of type $(2,1)$ with $J_{1}=\{1, i, j\}$ and $J_{2}=\{k, 2\}$ we have

$$
\begin{aligned}
\mathbf{1}_{\mathcal{C}_{J_{\lambda}}^{1}} & =\frac{1}{|a|^{3}} \frac{\left(f_{1 i j ; 1}\right)^{2}}{d_{1 i ; 1}^{1} d_{1 j ; 1}^{1} d_{i j ; 1}^{1}} \frac{\left(f_{2 k, 1 i ; 2}+f_{2 k, 1 j ; 2}+f_{2 k, i j ; 2}\right)}{d_{2 ; 2}^{1} d_{k ; 2}^{1}} \omega_{k i j}+\frac{a_{1}}{|a|^{3} a(1)} \frac{f_{1 i j ; 1}^{2}}{d_{1 i ; 1}^{1} d_{1 j ; 1}^{1} d_{i j ; 1}^{1}} \frac{f_{2 k, i j ; 2}}{d_{2 ; 2}^{1} d_{k ; 3}^{1}} \omega_{k i j} \\
& -\frac{a_{i}}{|a|^{3} a(i)} \frac{f_{1 i j ; 1}^{2}}{d_{1 i ; 1}^{i} d_{1 j ; 1}^{i} d_{i j ; 1}^{i}} \frac{f_{2 k, 1 j ; 2}}{d_{k ; 2}^{i} d_{2 ; 2}^{i}} \omega_{k 1 j}+\frac{a_{j}}{|a|^{3} a(j)} \frac{f_{1 i j ; 1}^{2}}{d_{1 i ; 1}^{j} d_{1 j ; 1}^{j} d_{i j ; 1}^{j}} \frac{f_{2 k, 1 i 2}}{d_{2 ; 2}^{j} d_{k ; 2}^{j}} \omega_{k 1 i}
\end{aligned}
$$

Type (2,1) with 1 in second auxillary arrangment: (Conjecture) For $\mathcal{C}_{J_{\lambda}}$ of type $(2,1)$ with $J_{1}=$ $\{k, i, j\}$ and $J_{2}=\{\ell, 1\}$ we have

$$
\begin{aligned}
\mathbf{1}_{\mathcal{C}_{J_{\lambda}}^{1}} & =\frac{a_{k}}{|a|^{3} a(k)} \frac{f_{k i j ; 1}^{2}}{d_{k i ; 1}^{k} d_{k j ; 1}^{k} d_{i j ; 1}^{k}} \frac{f_{1 \ell, i j ; 2}}{d_{1 ; 2}^{k} d_{k ; 3}^{k}} \omega_{\ell i j}-\frac{a_{i}}{|a|^{3} a(i)} \frac{f_{k i j ; 1}^{2}}{d_{k i ; 1}^{i} d_{k j ; 1}^{i} d_{i j ; 1}^{i}} \frac{f_{1 \ell, k j ; 2}}{d_{\ell ; 2}^{i} d_{1 ; 2}^{i}} \omega_{\ell k j} \\
& +\frac{a_{j}}{|a|^{3} a(j)} \frac{f_{k i j ; 1}^{2}}{d_{k i ; 1}^{j} d_{k j ; 1}^{j} d_{i j ; 1}^{j}} \frac{f_{1 \ell, k i ; 2}}{d_{1 ; 2}^{j} d_{\ell ; 2}^{j}} \omega_{\ell k i}
\end{aligned}
$$

Type (1,2) with 1 in first auxillary arrangement (Fact): For $C_{J_{\lambda}}$ of type $(1,2)$ with $J_{1}=\{1, h\}$ and $J_{2}=\{i, j, k\}$, we have

$$
\begin{aligned}
\mathbf{1}_{C_{J_{\lambda}}^{1}}= & \frac{1}{|a|^{3}} \frac{\left(f_{1 h ; 1}\right)}{d_{1 ; 1}^{1} d_{h ; 1}^{1}} \frac{\left(f_{i j k, 1 ; 2}^{2}+f_{i j k, h ; 2}^{2}+f_{i j k, 1 ; 2} f_{i j k, h ; 2}\right)}{d_{i j ; 2}^{1} d_{i k ; 2}^{1} d_{k j ; 2}^{1}} \omega_{h j k} \\
& +\frac{a_{h}}{|a|^{3} a(h)} \frac{f_{h 1 ; 1}}{d_{1 ; 1}^{h} d_{h ; 1}^{h}} \frac{f_{i j k, 1 ; 2}\left(2 f_{i j k, 1 ; 2}+f_{i j k, h ; 2}\right)}{d_{i j ; 2}^{h} d_{i k ; 2}^{h} d_{k j ; 2}^{h}} \omega_{1 k j} \\
& +\frac{a_{1}}{|a|^{3} a(1)} \frac{f_{1 h ; 1}}{d_{1 ; 1}^{1} d_{h ; 2}^{1}} \frac{f_{i j k, h ; 2}\left(2 f_{i j k, 1 ; 2}+f_{i j k, h ; 2}\right)}{d_{i j ; 2}^{1} d_{i k ; 2}^{1} d_{j k ; 2}^{1}} \omega_{h j k} \\
& +\frac{a_{1}}{|a|^{3} a(1)} \frac{f_{1 h ; 1}^{2}}{\left(d_{1 ; 1}^{1}\right)^{2}\left(d_{h ; 1}^{1}\right)^{2}} \frac{d_{i j k} f_{i j k, h ; 2}}{d_{i j ; 2}^{1} d_{i k ; 2}^{1} d_{j k ; 2}^{1}} \omega_{h j k} \\
& +\frac{a_{h}}{|a|^{3} a(h)}\left(\frac{a_{h}}{a(h)} \frac{f_{h 1 ; 1}}{d_{1 ; 1}^{h} d_{h ; 1}^{h}} \frac{-f_{i j k, 1 ; 2} f_{i j k, 1 ; 2}}{d_{i j ; 2}^{h} d_{i k ; 2}^{h} d_{j k ; 2}^{h}} \omega_{1 j k}+\frac{a_{1}}{a(1)} \frac{f_{1 h ; 1}}{d_{1 ; 1}^{1} d_{h ; 2}^{1}} \frac{-f_{i j k, 1 ; 2} f_{i j k, h ; 2}}{d_{i j ; 2}^{1} d_{i k ; 2}^{1} d_{j k ; 2}^{1}} \omega_{h j k}\right) \\
& +\frac{a_{1}}{|a|^{3} a(1)}\left(\frac{a_{h}}{a(h)} \frac{f_{h 1 ; 1}}{d_{1 ; 1}^{h} d_{h ; 1}^{h}} \frac{f_{i j k, 1 ; 2} f_{i j k, h ; 2}}{d_{i j ; 2}^{h} d_{i k ; 2}^{h} d_{j k ; 2}^{h}} \omega_{1 j k}+\frac{a_{1}}{a(1)} \frac{f_{1 h ; 1}}{d_{1 ; 1}^{1} d_{h ; 2}^{1}} \frac{f_{i j k, h ; 2} f_{i j k, h ; 2}}{d_{i j ; 2}^{1} d_{i k ; 2}^{1} d_{j k ; 2}^{1}} \omega_{h j k}\right) .
\end{aligned}
$$


Type $(1,2)$ with 1 in second auxillary arrangement (Conjecture): For $C_{J_{\lambda}}$ of type $(1,2)$ with $J_{1}=$ $\{i, j\}$ and $J_{2}=\{1, \ell, k\}$, we have

$$
\begin{aligned}
\mathbf{1}_{C_{J_{\lambda}}^{1}}= & \frac{a_{j}}{|a|^{3} a(j)} \frac{f_{j i ; 1}}{d_{i ; 1}^{j} d_{j ; 1}^{j}} \frac{f_{1 \ell k, i ; 2}\left(2 f_{1 \ell k, i ; 2}+f_{1 \ell k, j ; 2}\right)}{d_{1 \ell ; 2}^{j} d_{1 k ; 2}^{j} d_{\ell k ; 2}^{j}} \omega_{i k \ell} \\
& +\frac{a_{1}}{|a|^{3} a(1)} \frac{f_{i j ; 1}}{d_{j ; 1}^{i} d_{i ; 1}^{i}} \frac{f_{1 \ell k, j ; 2}\left(2 f_{1 \ell k, i ; 2}+f_{1 \ell k, j ; 2}\right)}{d_{1 \ell ; 2}^{i} d_{1 k ; 2}^{i} d_{\ell k ; 2}^{i}} \omega_{j k \ell} \\
& +\frac{a_{i}}{|a|^{3} a(i)} \frac{f_{i j ; 1}^{2}}{\left(d_{i ; 1}^{i}\right)^{2}\left(d_{i ; 1}^{i}\right)^{2}} \frac{d_{1 \ell k} f_{1 \ell k, j ; 2}}{d_{1 \ell ; 2}^{i} d_{1 k ; 2}^{i} d_{\ell k ; 2}^{i}} \omega_{j \ell k} \\
& +\frac{a_{j}}{|a|^{3} a(j)}\left(\frac{a_{j}}{a(j)} \frac{f_{j i ; 1}}{d_{i ; 1}^{j} d_{j ; 1}^{j}} \frac{-f_{1 \ell k, i ; 2} f_{1 \ell k, i ; 2}}{d_{1 \ell ; 2}^{j} d_{1 k ; 2}^{j} d_{\ell k ; 2}^{j}} \omega_{i \ell k}+\frac{a_{i}}{a(i)} \frac{f_{i j ; 1}}{d_{i ; 1}^{i} d_{j ; 2}^{i}} \frac{-f_{1 \ell k, i ; 2} f_{1 \ell k, j ; 2}}{d_{1 \ell ; 2}^{i} d_{1 k ; 2}^{i} d_{\ell k ; 2}^{i}} \omega_{j \ell k}\right) \\
& +\frac{a_{i}}{|a|^{3} a(i)}\left(\frac{a_{j}}{a(j)} \frac{f_{j i ; 1}}{d_{i ; 1}^{j} d_{j ; 1}^{j}} \frac{f_{1 \ell k, i ; 2} f_{1 \ell k, j ; 2}}{d_{1 \ell ; 2}^{j} d_{1 k ; 2}^{j} d_{\ell k ; 2}^{j}} \omega_{i \ell k}+\frac{a_{i}}{a(i)} \frac{f_{i j ; 1}}{d_{i ; 1}^{i} d_{j ; 2}^{i}} \frac{f_{1 \ell k, j ; 2} f_{1 \ell k, j ; 2}}{d_{1 \ell ; 2}^{i} d_{\ell k ; 2}^{i} d_{\ell k ; 2}^{i}} \omega_{j \ell k}\right) .
\end{aligned}
$$

Type (1,1,1): (Conjecture) We suspect this will involve a third layer of nesting, similar to the two layers of nesting seen up to this point. This implies there will be coefficients for weighted terms of the form $\frac{a_{i}}{a(i)} \frac{a_{j}}{a(j)} \frac{a_{k}}{a(k)}$ and that there will be places where three $f$ functions will be multiplied together. At this time, we do not know the form of this contribution.

Conjecture 5.3.1. The unit element of $A_{\varphi}$ can be expressed as

$$
\mathbf{1}=\sum_{\mathcal{C}_{J_{\lambda}}^{1}} \mathbf{1}_{\mathcal{C}_{J_{\lambda}}^{1}}
$$

where the $\mathbf{1}_{\mathcal{C}_{J_{\lambda}}^{1}}$ are the contributions from each elementary arrangement containing the line $H_{1}$ described above. 


\section{REFERENCES}

[AGV] V.I. Arnol'd, S.M. Gusein-Zade, A.N. Varchenko, Singularities of Differential Maps, Vol. I, Nauka, Moscow, 1982; also Birkhauser, 1985

[BB] M. Bayer, K. Brandt, Discriminantal arrangements, fiber polytopes and formality, J. Algebraic Combin. 6 (1997), no. 3, 229-246

[D] B. Dubrovin, Geometry of 2D topological field theories, Integrable Systems and Quantum Groups, ed. Francaviglia, M. and Greco, S.. Springer lecture notes in mathematics, 1620, 120-348

[GRTV] V. Gorbounov, R. Rimanyi, V. Tarasov, A. Varchenko, Quantum cohomology of the cotangent bundle of a flag variety as a Yangian Bethe algebra, arXiv:1204.5138, 1-44, Journal of Geometry and Physics (2013), pp. 56-86 DOI information: 10.1016/j.geomphys.2013.07.006

[GH] Ph. Griffiths, J. Harris, Principles of Algebraic Geometry, Wiley, 1994

[HV] C. Hertling, A. Varchenko, Potentials of a Frobenius like structure and $m$ bases of a vector space, arXiv: $1608.08423,1-18$

[NS] N. Nekrasov, S. Shatashvili, Quantum integrability and supersymmetric vacua, arXiv:0901.4748, Prog. Theor. Phys. Suppl. 177 (2009), 105-119,

[M] Y.I. Manin, Frobenius manifolds, quantum cohomology, and moduli spaces, American Mathematical Society Colloquium Publications, vol. 47, AMS, Providence, RI, 1999

[MTV1] E. Mukhin, V. Tarasov, A. Varchenko, Three sides of the geometric Langlands correspondence for $\mathrm{gl}_{N}$ Gaudin model and Bethe vector averaging maps, arXiv:0907.3266, Arrangements of hyperplanes - Sapporo 2009, 475-511, Adv. Stud. Pure Math., 62, Math. Soc. Japan, Tokyo, 2012

[MTV2] E. Mukhin, V. Tarasov, A. Varchenko, Bethe algebra of the $\mathfrak{g l}_{N+1}$ Gaudin model and algebra of functions on the critical set of the master function, New trends in quantum integrable systems, 307-324, World Sci. Publ., Hackensack, NJ, 2011

[OT1] P. Orlik, H. Terao, The number of critical points of a product of powers of linear functions, Invent. Math. 120 (1995), no. 1, 1-14

[OT2] P. Orlik, H. Terao, Arrangements and hypergeometric integrals, MSJ Memoir, 9 (2001), Math. Soc. Japan, Tokyo, ix + pp.112

[R] L. Rybnikov, A proof of the Gaudin Bethe Ansatz conjecture, arXiv:1608.04625, 1-15

[RV] N. Reshetikhin and A. Varchenko, Quasiclassical asymptotics of solutions to the KZ equations, Geometry, Topology and Physics for R. Bott, Intern. Press, 1995, 293-322

[Si] R. Silvotti, On a conjecture of Varchenko, Invent. Math. 126 (1996), no. 2, 235-248

[SV] V. Schechtman and A. Varchenko, Arrangements of Hyperplanes and Lie Algebra Homology, Invent. Math. 106 (1991), 139-194

[V1] A. Varchenko, Beta-Function of Euler, Vandermonde Determinant, Legendre Equation and Critical Values of Linear Functions of Configuration of Hyperplanes, I. Izv. Akademii Nauk USSR, Seriya Mat., 53:6 (1989), 1206-1235 
[V2] A. Varchenko, Multidimensional Hypergeometric Functions and Representation Theory of Lie Algebras and Quantum Groups, Advanced Series in Mathematical Physics, 21, World Scientific, 1995

[V3] A. Varchenko, Bethe Ansatz for Arrangements of Hyperplanes and the Gaudin Model, Mosc. Math. J. 6 (2006), no. 1, 195-210, 223-224

[V4] A. Varchenko, Quantum integrable model of an arrangement of hyperplanes, SIGMA Symmetry Integrability Geom. Methods Appl. 7 (2011), Paper 032, 55 pp.

[V5] A. Varchenko, Arrangements and Frobenius like structures, Annales de la faculte des sciences de Toulouse Ser. 6, 24 no. 1 (2015), p. 133-204

[V6] A. Varchenko, Critical set of the master function and characteristic variety of the associated GaussManin differential equations, Arnold Math. J. 1 (2015), no. 3, 253-282,

DOI $10.1007 / \mathrm{s} 40598-015-0020-8$, arXiv:1410.2438,1-24

[V7] A. Varchenko, On axioms of Frobenius like structure in the theory of arrangements, arXiv:1601.02208, Journal of Integrable Systems (2016) 00, 1-15, doi:10.1093/integr/xyw007 